UNIVERSIDAD AUTÓNOMA METROPOLITANA-Iztapalapa

\title{
Cubiertas Conservativas y Juegos Topológicos en Espacios de Funciones
}

Tesis que para obtener el grado de Doctor en Ciencias Matemáticas presenta el

M. en C. David Guerrero Sánchez

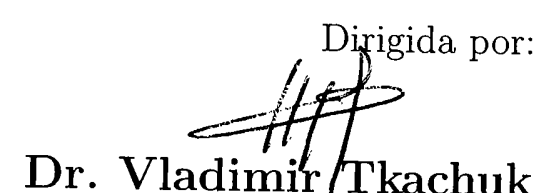

Junio 2012 
UNIVERSIDAD AUTÓNOMA METROPOLITANA-Iztapalapa

\title{
Cubiertas Conservativas y Juegos Topológicos en Espacios de Funciones
}

Tesis que para obtener el grado de Doctor en Ciencias Matemáticas presenta el

\author{
M. en C. David Guerrero Sánchez
}

Dirigida por:

Dr. Vladimir Tkachuk

Junio 2012 



\section{Ha menester}

Puesto que sin las condiciones necesarias es imposible alcanzar las conclusiones. Gracias a Guru Ram Das

A mi madre,

A mi padre,

A Pablo

A Siri Chand Kaur

Al Dr. Vladimir Tkachuk

Al Dr. Oleg Okunev

Al Dr. Ángel Tamariz

Al Dr. Mikhail Tkachenko

Al Dr. Richard Wilson

A la Universidad Autónoma Metropolitana

Al Consejo Nacional de Ciencia y Tecnología

A vos 


\section{Contenido}

$\begin{array}{ll}\text { Introducción } & 1\end{array}$

1. Preliminares 7

1.1. Notación y terminología . . . . . . . . . . . . . . 7

1.2. Invariantes cardinales y propiedades generales en $C_{p}(X) \ldots 11$

1.3. Las propiedades de $C_{p}(K)$ cuando $K$ es compacto . . . . . . 18

1.4. La propiedad de Lindelöf $\Sigma$ en $C_{p}(X) . \ldots \ldots \ldots . . . . .22$

1.5. Cubiertas conservativas generales y descomposiciones numerables de $C_{p}(X) \ldots \ldots \ldots \ldots \ldots \ldots$

2. Cubiertas conservativas de espacios de funciones 39

2.1. Observaciones generales . . . . . . . . . . . . . 40

2.2. Cubiertas conservativas cerradas de $C_{p}(X)$ y $C_{p}(X, \mathbb{I}) \ldots \ldots 45$

2.3. Cubiertas conservativas generales de $C_{p}(X)$ y $C_{p}(X, \mathbb{I}) \quad \ldots \quad 55$

2.4. Cubiertas conservativas de $C_{p}(K)$ para un espacio compacto $K \quad 60$

2.5. Juegos topológicos en $C_{p}(X) \ldots \ldots \ldots \ldots$

$\begin{array}{ll}\text { Problemas abiertos } & \mathbf{7 0}\end{array}$

$\begin{array}{ll}\text { Conclusiones } & 73\end{array}$

$\begin{array}{ll}\text { Bibliografía } & 75\end{array}$ 


\section{Introducción}

Uno de los objetivos principales de la Topología General es la clasificación y caracterización de los espacios topológicos. Para conseguirlo se han empleado técnicas, conocimientos y herramientas tanto de Topología General como de otras áreas de las Matemáticas. En virtud de un teorema fundamental de Nagata, para el caso de los espacios topológicos de Tychonoff, este estudio puede hacerse a través de la caracterización de las propiedades de los espacios de funciones reales dotados de la topología de convergencia puntual. Con este enfoque, se han desarrollado técnicas que conforman la rama del Álgebra Topológica denominada $C_{p}$-teoría. El presente texto se enmarca dentro de esta área del conocimiento. Una manera natural de estudiar un espacio topológico $X$ es representarlo como unión de sus subespacios comparativamente sencillos y aprovechar la estructura de dicha unión para obtener información de las propiedades de $X$. En ese sentido, lo primero que se tiene que averiguar es la conservación de las propiedades topológicas por las uniones finitas o numerables. Aquí, la mayoría de los resultados son bien conocidos y forman parte del folklore topológico. Por ejemplo, si $X$ es la unión finita de sus subespacios pseudocompactos entonces $X$ es pseudocompacto; si $X$ es unión finita de sus subespacios de $\pi$-peso numerable entonces $X$ tiene $\pi$-peso numerable. El $\pi$-peso y la pseudocompacidad ya no se preservan en uniones numerables, pero cualquier unión numerable de espacios cósmicos (es decir, los que tienen peso de red numerable) también es un espacio cósmico. Lo mismo es cierto para la propiedad de Lindelöf, la separabilidad, la $\sigma$-compacidad y muchas otras propiedades. Es muy común también considerar uniones de subespacios cerrados. Por ejemplo, la metrizabilidad ni siquiera se conserva por uniones de dos espacios; sin embargo, si $X$ es la unión finita de sus subespacios metrizables cerrados, entonces $X$ es metrizable. A veces, una estructura adicional en un espacio mejora dramáticamente sus propiedades en cuanto a uniones finitas o numerables. Un ejemplo clásico es el hecho de que cualquier gru- 
Introducción

po topológico representable como unión finita de sus subespacios primero numerables es metrizable. Los espacios de funciones tienen una estructura algebráica aún más rica que los grupos topológicos así que es de esperarse que en ellos se conserven más propiedades tanto por uniones finitas como por uniones numerables. Un estudio sistemático en este sentido se realizó en el artículo [Tk3] de Tkachuk. Él demostró, entre otras cosas, que si $C_{p}(X)$ es unión numerable de sus subespacios de carácter numerable entonces es metrizable. Análogamente, se demostró en [Tk3] que la estrechez, la propiedad de Fréchet-Urysohn y el pseudocarácter se conservan en $C_{p}(X)$ por uniones numerables. Además, si suponemos que $C_{p}(X)$ es unión numerable de subespacios cerrados realcompactos o hereditariamente normales, entonces $X$ es realcompacto o hereditariamente normal respectivamente. En el marco de la misma línea de investigación Casarrubias Segura estudió en [CS] las descomposiciones de $C_{p}(X)$ en uniones finitas. Él demostró, en particular, que si $C_{p}(X)$ es igual a la unión finita de una familia de sus subespacios paracompactos, entonces $C_{p}(X)$ es paracompacto. Adicionalmente, Casarrubias Segura establece, que si $n<\omega$ y $C_{p}(X)=\bigcup\{C i: i<n\}$ donde cada $C_{i}$ es realcompacto entonces $C_{p}(X)$ es realcompacto. Otro ejemplo del análisis de un espacio por medio de sus descomposiciones lo brindan los resultados de Tkachenko quien probó en el artículo [Tka] que para cualquier espacio $X=\bigcup\left\{X_{\alpha}: \alpha<\kappa\right\}$ donde $X_{\alpha} \subset X_{\beta}$ cuando $\alpha<\beta$ si tenemos un invariante cardinal $\phi \in\{$ número de Souslin, spread, número hereditario de Lindelöf, densidad hereditaria\} y $\phi(X)<\lambda$ para cada $\alpha<\kappa$; entonces $\phi(X) \leq \lambda$; si, además, $\kappa>\lambda$ entonces $\phi(X)<\lambda$.

El objetivo de esta tesis es estudiar cuándo un espacio de funciones tiene una cubierta conservativa (ver Sección 1.1) cuyos elementos poseen buenas propiedades. Evidentemente lo mismo se puede plantear para espacios generales. En este sentido se lograron algunos avances en los años 70-80 del siglo pasado; Potozny probó en [Po] que si un espacio $X$ admite una cubierta conservativa con subespacios compactos entonces $X$ es metacompacto. Por su parte, Smith y Telgarsky demostraron en [STe] que si un espacio $X$ es el $\sigma$-producto de espacios de Lindelöf y tiene una cubierta conservativa de subespacios compactos, entonces $X$ es Lindelöf. El capítulo preliminar del presente texto incluye el teorema de Terada-Yajima que establece que ningún subconjunto de tipo $G_{\delta}$ de un espacio numerablemente compacto admite una cubierta conservativa de subespacios densos en ninguna parte. En particular esto implica que si $\mathcal{C}$ es una cubierta conservativa de un espacio Čech-completo $X$ y los elementos de $\mathcal{C}$ son cerrados en $X$, entonces 
algún $C \in \mathcal{C}$ tiene interior no vacío. Este resultado es de gran utilidad en las secciones posteriores. El planteamiento general de la tesis puede definirse de la siguiente manera: dada una propiedad topológica $\mathcal{P}$; supongamos que $C_{p}(X)$ tiene una cubierta conservativa $\mathcal{F}$ de subespacios de $C_{p}(X)$ cuyos elementos tienen la propiedad $\mathcal{P}$. ¿Esto implica que $C_{p}(X)$ necesariamente tiene la propiedad $\mathcal{P}$ o alguna otra propiedad relacionada? Dedicaremos especial atención al caso cuando los elementos de la cubierta conservativa son cerrados en $C_{p}(X)$. Para la mayoría de las propiedades topológicas $\mathcal{P}$ si $C_{p}(X)$ tiene una cubierta cerrada numerable cuyos elementos tienen $\mathcal{P}$, entonces $C_{p}(X)$ también posee una cubierta conservativa cuyos elementos tienen $\mathcal{P}$. De modo que la información que hemos obtenido sobre cubiertas cerradas conservativas generalizan los resultados de Tkachuk sobre uniones cerradas numerables en [Tk1] y [Tk3]. El capítulo 1 de la tesis es preliminar; aquí se presentan las definiciones y resultados propios de la Topología General que se utilizan en las demostraciones de los enunciados del capítulo 2. En particular, en la Sección 1.5 citamos algunos resultados clásicos sobre los espacios de funciones que admiten una cubierta numerable por subespacios con "buenas" propiedades. El capítulo 2 consiste en los resultados originales de la tesis. Comenzamos con el caso de cubiertas localmente finitas demostrando que esta situación se reduce al caso de cubiertas finitas. El segundo caso que consideramos es la existencia de cubiertas conservativas conformadas por subespacios compactos, o bien numerablemente compactos de espacios $C_{p}(X)$ para un espacio arbitrario $X$; el resultado principal de la sección 2.1 es demostrar que en este caso $X$ tiene que ser finito. En la sección 2.2 el resultado principal es el Teorema 2.2.8 que afirma que dado un espacio $X$ y una propiedad $\mathcal{P}$ hereditaria con respecto a subespacios cerrados, si $C_{p}(X,[0,1])$ tiene una cubierta conservativa cerrada $\mathcal{C}$ tal que cada $C \in \mathcal{C}$ tiene $\mathcal{P}$ entonces $C_{p}(X,[0,1])$ también tiene la propiedad $\mathcal{P}$. Esto implica, en particular, que para cualquier espacio de Tychonoff $X$, el espacio $C_{p}(X,[0,1])$ es Lindelöf si y sólo si $C_{p}(X,[0,1])$ tiene una cubierta conservativa cerrada de subespacios de Lindelöf. En la misma sección también se estudian en $C_{p}(X)$ las cubiertas conservativas cerradas cuyos elementos son de Lindelöf o Lindelöf $\Sigma$. A sabiendas de que el espacio $C_{p}(X,[0,1])$ tiene la propiedad correspondiente, la conjetura principal es que en este caso el mismo $C_{p}(X)$ será Lindelöf o Lindelöf $\Sigma$ respectivamente. En esta dirección, se demuestran algunos resultados positivos particulares, la mayoría de los cuales son generalizaciones de teoremas clásicos sobre los espacios $X$ tales que $C_{p}(X)$ es Lindelöf o Lindelöf $\Sigma$. En la sección 2.3 estudiamos las cubiertas conservativas no necesariamen- 
Introducción

te cerradas de los espacios de funciones con la topología de la convergencia puntual. El Teorema 2.3.2 establece que si $\mathcal{P}$ es una propiedad $\sigma$-aditiva, invariante bajo imágenes continuas y todos los espacios unipuntuales tienen $\mathcal{P}$, entonces el espacio $C_{p}(X)\left(\right.$ o $\left.C_{p}(X,[0,1])\right)$ tiene una cubierta conservativa cuyos elementos tienen $\mathcal{P}$ si y sólo si $C_{p}(X)$ tiene un subespacio denso con la propiedad $\mathcal{P}$. Este es el resultado principal de la sección 2.3 y nos permite probar, por ejemplo, que para cualquier espacio compacto $K$; el espacio $C_{p}(K)$ tiene una cubierta conservativa con subespacios $\sigma$-compactos si y sólo si el compacto $K$ es Eberlein. El resultado principal de la sección 2.4 es el Ejemplo 2.4.8 que muestra un espacio $\sigma$-compacto $X$ tal que $C_{p}(X)$ contiene un subespacio denso $\sigma$-compacto, pero $C_{p}(X)$ no es Lindelöf. Este ejemplo prueba que un espacio $C_{p}(X)$ puede no ser Lindelöf y aun así tener una cubierta conservativa de subespacios (no cerrados) Lindelöf $\Sigma$. La misma sección también incluye el Teorema 2.4.1 que establece que si $K$ es un espacio compacto tal que $w(K)>\kappa$ entonces $C_{p}(K)$ no tiene una cubierta conservativa compuesta por subespacios de cardinalidad $\kappa$ : Esto permite demostrar que un espacio compacto $K$ es metrizable si y sólo si $C_{p}(K)$ tiene una cubierta conservativa de subespacios separables. En la sección 2.5 se presenta el siguiente juego topológico: en un espacio $Y$, consideremos una familia $\mathcal{C}$ de subconjuntos de $Y$. Definimos el juego $\mathcal{G}(\mathcal{C}, Y)$ para dos jugadores $I$ y $I I$ quienes alternan jugadas de modo que en la jugada número $n$, el jugador $I$ elige $C_{n} \in \mathcal{C}$ y el jugador $I I$ elige una vecindad abierta $U_{n}$ del conjunto $C_{n}$. Cuando la $n$-ésima jugada para cada $n \in \omega$ ha sido efectuda, el juego termina. El jugador $I$ gana si $X=\bigcup\left\{U_{n}: n \in \omega\right\}$; en caso contrario el ganador es el jugador $I I$. El resultado principal de esta sección es el Teorema 2.5.4 que establece que dado un espacio no vacío $X$, para el espacio $Y=C_{p}(X,[0,1])$ (o $\left.Y=C_{p}(X)\right)$ y la familia $\mathcal{F}=\{F \subset Y: F$ es denso en ninguna parte en $\left.C_{u}(X,[0,1])\right\}$ (o $\mathcal{F}=\left\{F \subset Y: F\right.$ es denso en ninguna parte en $\left.C_{u}(X)\right\}$ ) el jugador $I I$ tiene una estrategia ganadora en el juego $\mathcal{G}(\mathcal{F}, Y)$. Este resultado nos permite demostrar que si $\mathcal{C}$ es la familia de todos los subespacios $\sigma$-compactos de $C_{p}(X)$ que son cerrados en $C_{u}(X)$ y el jugador $I$ tiene una estrategia ganadora sobre $C_{p}(X)$ en el juego $\mathcal{G}\left(\mathcal{C}, C_{p}(X)\right)$; entonces el espacio $X$ es finito. En el apartado de problemas abiertos se enuncian algunas de las preguntas que surgen de manera natural en el estudio de los temas abordados en el presente texto y que aún no se han podido responder. En particular, en el Problema 1 se plantea si $C_{p}(X)$ es necesariamente Lindelöf cuando admite una cubierta conservativa de subespacios cerrados de Lindelöf. La pregunta análoga, correspondiente al caso en el que $C_{p}(X)$ tiene una cubierta con- 
servativa cerrada de subespacios Lindelöf $\Sigma$, se presenta en el Problema 2. Finalmente, en el Problema 10 se propone estudiar si la numerabilidad del peso se preserva bajo uniones conservativas en espacios de funciones. Cabe mencionar que en la tesis se resuelven 8 problemas publicados y se generalizan algunos resultados publicados en artículos de Tkachuk, Arhangel'skii, Casarrubias y Okunev. 


\section{Capítulo 1}

\section{Preliminares}

\subsection{Notación y terminología}

Salvo que se indique otra cosa, todos los espacios que aparecen en el texto pertenecen a la clase de los espacios de Tychonoff. La notación empleada es la usual. Las letras $X, Y, Z$ denotan, en general, espacios topológicos, mientras que $x, y, z, p$ se usan para hacer referencia a puntos de algún espacio. Los ordinales son representados por las letras griegas minúsculas $\alpha, \beta, \gamma, \lambda$, $\xi$. Para un espacio $X$ la familia de todos los subconjuntos de $X$ se denota por $\exp (X)$, la familia de todos los subconjuntos finitos de $X$ se denota por $[X]^{<\omega}$, para designar la topología de un espacio $X$ se escribe $\tau(X)$ y $\tau^{*}(X)$ es la familia de todos los abiertos no vacíos de $X$. La familia de todos los abiertos de $X$ que contienen al punto $x$ se designa $\tau(x, X)$; y si $F \subset X$, la familia de abiertos de $X$ que contienen al conjunto $F$ se denota $\tau(F, X)$. Un mapeo $\varphi: X \rightarrow \exp (Y)$ se llama una multifunción superiormente semicontinua o un mapeo superiormente semicontinuo si para cualquier $U \in \tau(Y)$, el conjunto $\varphi_{u}^{-1}(U)$ definido por $\varphi_{u}^{-1}(U):=\{x \in X: \varphi(x) \subset U\} \in \tau(X)$. Si $\varphi(x)$ es un subconjunto compacto de $Y$ para cada $x \in X$ entonces el mapeo $\varphi$ se llama compacto-valuado. Si $Y=\bigcup\{\varphi(x): x \in X\}$ entonces la multifunción $\varphi$ se llama sobreyectiva.

La letra $\mathbb{R}$ simboliza la recta real con la topología usual, el intervalo $[0,1] \subset \mathbb{R}$ se representa por $\mathbb{I}$. La letra $\omega$ representa el primer cardinal infinito y $\omega_{1}$ se refiere al primer cardinal no numerable. Dado un espacio $X$ y $A \subset X$, la cerradura de $A$ en $X$ se designa por $\bar{A}$ y si $A \subset D \subset X$, para denotar la cerradura de $A$ en $D$ se escribe $\bar{A}^{D}$ y el interior relativo de $A$ en $D$ se 
Preliminares

denota por $\operatorname{int}_{D}(A)$. Dado un cardinal $\kappa$ sea $\Sigma(\kappa)=\left\{x \in \mathbb{R}^{\kappa}\right.$ : el conjunto $x^{-1}(\mathbb{R} \backslash\{0\})$ es numerable $\}$. El espacio $\Sigma(\kappa)$ se llama el $\Sigma$-producto de $\kappa$ rectas reales. Decimos que $\mathcal{C}$ es una $\omega$-cubierta de un espacio $X$ si para cada conjunto finito $A \subset X$ existe $U \in \mathcal{C}$ tal que $A \subset U$.

El espacio de todas las funciones continuas de un espacio $X$ en un espacio $Y$ se denota por $C(X, Y)$. El espacio $C(X, \mathbb{R})$ se abrevia por $C(X)$ mientras que $C^{*}(X) \subset C(X, \mathbb{R})$ es el conjunto de todas las funciones continuas con valores reales acotadas del espacio $X$. El espacio $C(X, Y)$ dotado de la topología heredada del producto $Y^{X}$, se denota por $C_{p}(X, Y)$. El espacio $C_{p}(X, \mathbb{R})$ se abrevia por $C_{p}(X)$. Dada $f \in C_{p}(X)$, un conjunto finito $A \subset X$, y un número real positivo $\varepsilon$, definimos el conjunto $O(f, A, \varepsilon)=$ $\left\{g \in C_{p}(X):|g(x)-f(x)|<\varepsilon\right.$ para cada $\left.x \in A\right\}$. Para cualesquiera puntos $x_{1}, \ldots, x_{n}$ de un espacio $X$ y conjuntos $O_{1}, \ldots, O_{n}$ abiertos en $\mathbb{R}$ el conjunto $\left[x_{1}, \ldots, x_{n} ; O_{1}, \ldots, O_{n}\right]=\left\{f \in C_{p}(X): f\left(x_{i}\right) \in O_{i}\right.$ para cada $\left.i=1, \ldots, n\right\}$ es abierto en $C_{p}(X)$. De hecho tanto la familia de los conjuntos de la forma $O(f, A, \varepsilon)$ como la familia de los conjuntos de la forma $\left[x_{1}, \ldots, x_{n} ; O_{1}, \ldots, O_{n}\right]$ son bases de la topología de $C_{p}(X)$. Para todo $f \in C_{p}(X, Y)$, definimos el mapeo dual $f^{*}: C_{p}(Y) \rightarrow C_{p}(X)$ por $f^{*}(g)=g \circ f$ para cada $g \in C_{p}(Y)$. Para cualquier $X \subset C_{p}(Y)$ definimos el producto diagonal $\varphi: Y \rightarrow C_{p}(X)$ de los elementos de $X$ de la siguiente manera: para cada $y \in Y$ y $f \in X$ hacemos $\varphi(y)(f)=f(y)$ for any $p \in K$ and $f \in X$.

Por otra parte, $C_{u}(X)$ denota al espacio $C(X)$ con la topología de la convergencia uniforme. Para cualesquiera $f, g \in C_{u}(X)$ definimos $d(f, g)=$ $\operatorname{mín}\{1, \sup \{|f(x)-g(x)|: x \in X\}\}$. Para todo $r>0$ la bola abierta de radio $r$ centrada en $f$ es el conjunto $B(f, r)=\left\{g \in C_{u}(X): d(f, g)<r\right\} \in$ $\tau\left(C_{u}(X)\right)$. La familia $\{B(f, r): f \in C(X), r>0\}$ es una base de $C_{u}(X)$. Nótese que la topología del espacio $C_{u}(X)$ es más fina que la topología de $C_{p}(X)$. Decimos que una familia $\mathcal{F} \subset C(X)$ separa los puntos del espacio $X$ si para cualesquiera $x, y \in X$ existe $f \in \mathcal{F}$ tal que $f(x) \neq f(y)$. Un mapeo continuo $f: X \rightarrow Y$ se llama $\mathbb{R}$-cociente si para cada función $g: Y \rightarrow \mathbb{R}$ la continuidad de $g \circ f$ implica la continuidad de $g$. Una biyección continua se llama condensación, y si $f: X \rightarrow Y$ es una condensación, decimos que $X$ se condensa sobre $Y$.

Dado un espacio $X$, una familia $\mathcal{N}$ se llama una red de $X$ si, para cualquier $U \in \tau(X)$, existe $\mathcal{N}^{\prime} \subset \mathcal{N}$ tal que $\bigcup \mathcal{N}^{\prime}=U$. El cardinal $n w(X)=\min \{|\mathcal{N}|:$ $\mathcal{N}$ es una red de $X\}$ es el peso de red de $X$. El cardinal $l(X)=\min \{\kappa:$ toda cubierta abierta de $X$ tiene una subcubierta de cardinalidad $\leq \kappa\}$ se llama el número de Lindelöf de $X$. Para cada $A \subset X$, sea $\psi(A, X)=\operatorname{mín}\{|\mathcal{U}|$ : 
$\mathcal{U} \subset \tau(X)$ and $\bigcap \mathcal{U}=A\}$. El cardinal $\psi(A, X)$ se llama el pseudocarácter de $A$ en $X$. Si $A=\{x\}$ escribimos $\psi(x, X)$ en lugar de $\psi(\{x\}, X)$. El cardinal $\psi(X)=\sup \{\psi(x, X): x \in X\}$ es el pseudocarácter de $X$. El conjunto $\Delta_{X}=\{(x, x): x \in X\} \subset X \times X$ es la diagonal de $X$. El número diagonal de un espacio $X$ es el cardinal $\Delta(X)=\psi\left(\Delta_{X}, X \times X\right)$.

Decimos que un espacio $X$ tiene diagonal pequeña si para cada conjunto no numerable $A \subset(X \times X) \backslash \Delta_{X}$, existe una vecindad $U$ de $\Delta$ tal que $|A \backslash U|>\omega$.

Un cardinal $\kappa$ es un calibre de un espacio $X$ si para cualquier familia $\mathcal{U} \subset \tau^{*}(X)$ con $|\mathcal{U}|=\kappa$ existe $\mathcal{V} \subset \mathcal{U}$ tal que $|\mathcal{V}|=\kappa$ y $\cap \mathcal{V} \neq \emptyset$. El cardinal $s(X)=\sup \{|D|: D \subset X$ es discreto $\}$ se llama el spread del espacio $X$. Por otra parte, el cardinal $i w(X)=\min \{|\kappa|: X$ se condensa sobre un espacio de peso $\kappa$ \} se llama el $i$-peso de $X$. Dado un cardinal $\kappa$ y $A \subset X$ sea $[A]_{\kappa}=\bigcup\{\bar{B}: B \subset A$ y $|B| \leq \kappa\}$. Definimos $t(X)=\min \left\{\kappa: \bar{A}=[A]_{\kappa}\right.$ para cada $A \subset X\}$. El cardinal $t(X)$ se llama la estrechez del espacio $X$.

Dado un espacio $X$, una familia $\mathcal{F} \subset \exp (X)$ se llama $T_{0}$-separadora si para cualesquiera puntos distintos $x, y \in X$ existe $V \in \mathcal{V}$ tal que $V \cap\{x, y\}$ es un conjunto unipuntual. Una familia $\mathcal{F} \subset \exp (X)$ se llama conservativa si para cualquier $\mathcal{G} \subset \mathcal{F}$ sucede que $\overline{\bigcup \mathcal{G}}=\bigcup\{\bar{G}: G \in \mathcal{G}\}$.

Si un espacio $X$ es homeomorfo a un espacio $Y$ escribimos $X \simeq Y$. Un espacio $\sigma$-compacto ( $\sigma$-numerablemente compacto) es la unión numerable de espacios compactos (numerablemente compactos). Un espacio es cósmico si tiene una red numerable. Dado un cardinal $\kappa$, decimos que un espacio es $\kappa$-monolítico si $n w(\bar{A}) \leq \kappa$ para cada $A \subset X$ tal que $|A| \leq \kappa$. En particular un espacio $X$ es $\omega$-monolítico si cada cada subespacio separable de $X$ es cósmico. Un subconjunto $A$ de un espacio $X$ es de tipo $G_{\delta}$ si existe una familia numerable $\mathcal{U} \subset \tau(X)$ tal que $A=\bigcap \mathcal{U}$. Un espacio $X$ se llama $P$-espacio si cada conjunto de tipo $G_{\delta}$ es abierto en $X$. Un espacio $X$ es monolítico si, para cada $A \subset X$, tenemos que $d(A)=n w(A)$. Un espacio $X$ se llama estable si para cada imagen continua $Y$ de $X$ tenemos que $i w(Y)=n w(Y)$. Un subconjunto $A \subset X$ es $C$-encajado en $X$ si para cualquier mapeo continuo $f: A \rightarrow \mathbb{R}$ existe $g \in C_{p}(X)$ tal que $g \mid A=f$. Si cada $f \in C^{*}(A)$ se puede extender a un mapeo continuo $g: X \rightarrow \mathbb{R}$, entonces el conjunto $A$ se llama $C^{*}$-encajado.

Para cualquier espacio $X$ la clase $\mathcal{E}(X)$ está compuesta por todas las imágenes continuas del producto de $X$ con algún espacio compacto. Una clase $\mathcal{P}$ de espacios topológicos se llama $k$-dirigida si para cualesquiera $Y, Z \in \mathcal{P}$ se cumple que $Y \times Z \in \mathcal{P}$ y $\mathcal{E}(Y) \in \mathcal{P}$. La clase $\mathcal{P}$ se llama $\omega$-perfecta si es 
cerrada bajo subespacios cerrados, y $Z \in \mathcal{P}$ implica que tanto $(Z \times \omega)^{\omega} \in \mathcal{P}$ como $\mathcal{E}(Z) \in \mathcal{P}$. Una cubierta de un espacio $X$ se llama compacta (cerrada) si sus elementos son subespacios compactos (cerrados) de $X$. Para un espacio $X$ decimos que una familia $\mathcal{F} \subset \exp (X)$ es una red con respecto a una familia $\mathcal{G} \subset \exp (X)$ si dado cualquier $G \in \mathcal{G}$, para todo $U \in \tau(G, X)$ existe $F \in \mathcal{F}$ tal que $G \subset F \subset U$. Un espacio Lindelöf $\Sigma$ es aquel que tiene una red numerable con respecto a una cubierta compacta.

El resto de la terminología empleada en este texto es estándar y se encuentra definida en los libros [Ar2], [Tk6] y [En], así como en los artículos [Ho] y [Ju]. 


\subsection{Invariantes cardinales y propiedades ge- nerales en $C_{p}(X)$}

En esta sección se presentan algunos de los resultados más útiles de la $C_{p}$-teoría que se usarán en las demostraciones del Capítulo 2. El estudio de los espacios de funciones que aquí se presenta se realiza aprovechando sus estructuras algebraicas adicionales. En particular, gracias a su estructura de anillos topológicos, es posible verificar que el comportamiento de los invariantes cardinales en los espacios $C_{p}(X)$ es mejor que en los espacios topológicos generales. Un ejemplo de esto se puede encontrar en el resultado del Teorema 1.2.6 que implica la igualdad $\chi\left(C_{p}(X)\right)=w\left(C_{p}(X)\right)$ que en general no ocurre en espacios arbitrarios.

Proposición 1.2.1. [Tk6, Fact 3 de S.154], [Ter] Si X es un espacio pseudocompacto y $Y$ es segundo numerable, entonces cualquier mapeo continuo suprayectivo $f: X \rightarrow Y$ es $\mathbb{R}$-cociente.

Demostración. Supongamos que $f: X \rightarrow Y$ es un mapeo continuo suprayectivo y tomemos cualquier función $g: Y \rightarrow \mathbb{R}$ tal que la composición $g \circ f$ es continua. Fijemos cualquier cerrado $F \subset \mathbb{R}$ para probar que $G=g^{-1}(F)$ es cerrado en $Y$. Para cualquier $x \in \mathbb{R}$, definimos $d_{F}(x)=$ ínf $\{|x-y|: y \in F\}$. La función $d_{f}: \mathbb{R} \rightarrow[0,+\infty)$ es continua y por lo tanto el conjunto $U_{n}=d_{F}^{-1}\left(\left[0, \frac{1}{n}\right)\right)$ es abierto en $\mathbb{R}$ y contiene a $F$. Además $\bar{U}_{n+1} \subset d_{F}^{-1}\left(\left[0, \frac{1}{n+1}\right]\right) \subset d_{F}^{-1}\left(\left[0, \frac{1}{n}\right)\right)=U_{n}$ para cada $n \in \mathbb{N}$. En consecuencia, $F=\bigcap\left\{U_{n}: n \in \mathbb{N}\right\}=\bigcap\left\{\bar{U}_{n}: n \in \mathbb{N}\right\}$. El conjunto $H=(g \circ f)^{-1}(F)$ es cerrado en $X$ y $H=\bigcap\left\{V_{n}: n \in \mathbb{N}\right\}=\bigcap\left\{\bar{V}_{n}: n \in \mathbb{N}\right\}$ donde $V_{n}=(g \circ f)^{-1}\left(U_{n}\right)$ para todo $n$ natural. Por lo tanto $G=\bigcap\left\{f\left(\bar{V}_{n}\right): n \in \mathbb{N}\right\}$.

Notemos que cada $\bar{V}_{n}$ es un espacio pseudocompacto. En efecto, si $\gamma$ es una familia localmente finita de abiertos no vacíos de $\bar{V}_{n}$ entonces $\gamma^{\prime}=$ $\left.\left\{V \cap V_{n}\right): V \in \gamma\right\}$ es una familia localmente finita de abiertos no vacíos del espacio $X$. Como $X$ es pseudocompacto, la familia $\gamma^{\prime}$ es finita lo que implica que $\gamma$ también lo es y por lo tanto $\bar{V}_{n}$ es un espacio pseudocompacto. Cada conjunto $f\left(\bar{V}_{n}\right)$ es compacto ya que es Lindelöf y pseudocompacto. Por este motivo $f\left(\bar{V}_{n}\right)$ es cerrado en el espacio $Y$ para cada $n \in \mathbb{N}$. Finalmente el conjunto $G$ es cerrado por ser intersección de cerrados lo cual muestra que la función $g$ es continua y el mapeo continuo $f$ es $\mathbb{R}$-cociente. 
Proposición 1.2.2. Si $X$ es un espacio que contiene una familia discreta $\mathcal{U} \subset \tau^{*}(X)$, entonces existe un espacio discreto $D$ tal que $|D|=|\mathcal{U}|$ y $\mathbb{R}^{D}$ es factor y retracto de $C_{p}(X)$.

Demostración. Tomemos una familia discreta $\mathcal{U}=\left\{U_{\alpha}: \alpha \in \kappa\right\} \subset \tau^{*}(X)$ elijamos un punto $d_{\alpha} \in U_{\alpha}$ para cada $\alpha \in \kappa$ y sea $D=\left\{d_{\alpha}: \alpha \in \kappa\right\}$; este conjunto es cerrado y discreto en $X$. Para cada $\alpha \in \kappa$ existe una función $f_{\alpha} \in C(X,[0,1])$ tal que $f_{\alpha}\left(d_{\alpha}\right)=1$ y $f_{\alpha}\left(X \backslash U_{\alpha}\right) \subset\{0\}$. Para cada $\xi \in \mathbb{R}^{D}$ definimos la función $e_{\xi} \in \mathbb{R}^{X}$ en cada $x \in X$ por $e_{\xi}(x)=\sum_{\alpha \in \kappa} f_{\alpha}(x) \xi\left(d_{\alpha}\right)$. Esta función es continua puesto que para cada $x \in X$ existe $U \in \tau(x, X)$ que interseca a lo más a un solo elemento $U_{\alpha}$ de la familia $\mathcal{U}$, por lo que $\beta \neq \alpha$ implica $f_{\beta}(U) \subset\{0\}$ de manera que $e_{\xi}\left|U=\xi\left(d_{\alpha}\right) \cdot f_{\alpha}\right| U$ así que la continuidad de $\xi\left(d_{\alpha}\right) \cdot f_{\alpha}$ garantiza la continuidad de $e_{\xi}$ en el punto $x$.

De modo que tenemos el mapeo $e: \mathbb{R}^{D} \rightarrow C_{p}(X)$ definido por medio de la igualdad $e(\xi)=e_{\xi}$ para cada $\xi \in \mathbb{R}^{D}$. Fijemos $x \in X$ y sea $\pi_{x}: C_{p}(X) \rightarrow \mathbb{R}$ la proyección sobre el factor determinado por $x$. Para ver que $\pi_{x} \circ e: \mathbb{R}^{D} \rightarrow \mathbb{R}$ es continua, supongamos primero que $x \notin \bigcup\left\{U_{\alpha}: \alpha \in \kappa\right\}$ lo cual implica que para toda $\xi \in \mathbb{R}^{D}$ tenemos que $\left(\pi_{x} \circ e\right)(\xi)=e_{\xi}(x)=\sum_{\alpha \in \kappa} f_{\alpha}(x) \xi\left(d_{\alpha}\right)=0$ por lo que la función $\pi_{x}$ o e es continua. Si existe $\alpha \in \kappa$ tal que $x \in U_{\alpha}$ entonces para toda $\xi \in \mathbb{R}^{D}$ tenemos que $\left(\pi_{x} \circ e\right)(\xi)=e_{\xi}(x)=f_{\alpha}(x) \xi\left(d_{\alpha}\right)$ así que $\pi_{x} \circ e$ es una función continua. Esto muestra que el mapeo $e$ es continuo.

Más aún, para cualesquiera $\xi, \xi^{\prime} \in \mathbb{R}^{D}$ se cumple la igualdad $e_{\xi+\xi^{\prime}}=$ $e_{\xi}+e_{\xi^{\prime}}$. En efecto, si $\xi, \xi^{\prime}$ son elementos de $\mathbb{R}^{D}$, entonces

$e_{\xi+\xi^{\prime}}=\sum_{\alpha \in \kappa}\left(\xi+\xi^{\prime}\right)\left(d_{\alpha}\right) f_{\alpha}=\sum_{\alpha \in \kappa} \xi\left(d_{\alpha}\right) \cdot f_{\alpha}+\sum_{\alpha \in \kappa} \xi^{\prime}\left(d_{\alpha}\right) \cdot f_{\alpha}=e_{\xi}+e_{\xi^{\prime}}$. Además si $r \in \mathbb{R}$ entonces $e_{r \xi}=r e_{\xi}$. Si $\xi \neq \xi^{\prime}$ entonces existe un $\alpha \in \kappa$ tal que $\xi\left(d_{\alpha}\right) \neq \xi^{\prime}\left(d_{\alpha}\right)$ lo que implica que $e_{\xi}\left(d_{\alpha}\right)=\sum_{\alpha \in \kappa} \xi\left(d_{\alpha}\right) f_{\alpha}\left(d_{\alpha}\right)=\xi\left(d_{\alpha}\right)$ difiere de $\xi^{\prime}\left(d_{\alpha}\right)=e_{\xi^{\prime}}\left(d_{\alpha}\right)$ y por lo tanto $e$ es una inyección.

Para ver que $e$ es una inmersión hagamos $L=e\left(\mathbb{R}^{D}\right)$ y consideremos el mapeo $e^{-1}: L \rightarrow \mathbb{R}^{D}$. Con el fin de verificar la continuidad de $e^{-1}$ fijemos $\alpha \in \kappa$ y tomemos la proyección $\rho_{\alpha}: \mathbb{R}^{D} \rightarrow \mathbb{R}$ sobre el factor determinado por $d_{\alpha}$. Respecto de la composición $\rho_{\alpha} \circ e^{-1}: L \rightarrow \mathbb{R}$ observemos que para cualquier $f \in L$ tenemos la igualdad $\left(\rho_{\alpha} \circ e^{-1}\right)(f)=\left(\rho_{\alpha} \circ e^{-1}\right)\left(e_{\xi}\right)=\xi\left(d_{\alpha}\right)=$ $f\left(d_{\alpha}\right)=e_{d_{\alpha}}(f)$ así que $\rho_{\alpha} \circ e^{-1}$ es continua. Por lo tanto $e$ es una inmersión.

Por construcción, $L$ es un subespacio lineal de $C_{p}(X)$. Consideremos el conjunto $M=\left\{f \in C_{p}(X):\left.f\right|_{D}=0\right\}$; mostraremos que $C_{p}(X)$ es homeomorfo a $L \times M$. Para cada $f \in C_{p}(X)$ sea $\zeta_{f}=\left.f\right|_{D}$, esto implica que $\left.e_{\zeta_{f}}\right|_{D}=\left.f\right|_{D}$ por lo cual $\left.\left(f-e_{\zeta_{f}}\right)\right|_{D}(d)=0$ para cada $d \in D$, así que $e_{\zeta_{f}} \in L$ 
y $\left(f-e_{\zeta_{f}}\right) \in M$. Ahora definimos el mapeo $u: C_{p}(X) \rightarrow L \times M$ por medio de la fórmula $u(f)=\left(e_{\zeta_{f}}, f-e_{\zeta_{f}}\right)$ para cada $f \in C_{p}(X)$. El mapeo $u$ es continuo y si hacemos $v(f, g)=f+g$ para cada $(f, g) \in L \times M$, entonces el mapeo $v: L \times M \rightarrow C_{p}(X)$ es continuo y es fácil ver que $v$ es el inverso de $u$ de modo que $u$ es un homeomorfismo lineal. Finalmente el espacio $L$ es homeomorfo a $\mathbb{R}^{D}$ que es homeomorfo a $\mathbb{R}^{\kappa}$ así que la proyección de $C_{p}(X)$ sobre $L$ es una retracción de $C_{p}(X)$ sobre un espacio homeomorfo a $\mathbb{R}^{\kappa}$.

Teorema 1.2.3. [Tk6, Problem 109] Cualquier producto de espacios separables tiene número de Souslin numerable. En particular el número de Souslin de $\mathbb{R}^{A}$ es numerable para cualquier conjunto $A$.

Proposición 1.2.4. [Tk6, Problem 110] Si Y es un subespacio denso de un espacio $X$ entonces $c(Y)=c(X)$.

Demostración. Supongamos que $\gamma$ es una familia disjunta de subconjuntos abiertos no vacíos de $X$. Entonces $\mu=\{U \cap Y: U \in \gamma\}$ es una familia disjunta de subconjuntos abiertos no vacíos de $Y$ con $|\mu|=|\gamma|$. Por lo tanto, tenemos que $c(X) \leq c(Y)$. Por otra parte, si $\mu$ es una familia disjunta de subconjuntos abiertos no vacíos de $Y$ entonces, para cada $U \in \mu$, existe $W(U) \in \tau(X)$ con $W(U) \cap Y=U$. Observemos que cualquier conjunto abierto no vacío de $X$ interseca a $Y$ puesto que $Y$ es denso en $X$. Ahora, si $W(U) \cap W(V) \neq \varnothing$ entonces el subconjunto abierto no vacío $W(U) \cap W(V)$ debe intersecar a $Y$, de modo que $\varnothing \neq W(U) \cap W(V) \cap Y=U \cap V$ lo cual es una contradicción que prueba que la familia $\gamma=\{W(U): U \in \mu\}$ es disjunta. La igualdad $|\mu|=|\gamma|$ muestra que $c(Y) \leq c(X)$.

Proposición 1.2.5. [Tk6, Problem 111] Para todo espacio $X$ el espacio $C_{p}(X)$ es denso en $\mathbb{R}^{X}$ y por lo tanto el número de Souslin de $C_{p}(X)$ es numerable.

Demostración. Para verificar que $C_{p}(X)$ es denso en $\mathbb{R}^{X}$, tomemos cualquier subconjunto abierto no vacío estándar $U=\Pi_{x \in X} U_{x}$ del espacio $\mathbb{R}^{X}$. El conjunto $A=\left\{x \in X: U_{x} \neq \mathbb{R}\right\}$ es finito y es posible encontrar $r_{x} \in U_{x}$ para cada $x \in A$. Puesto que $X$ es un espacio de Tychonoff existe $f \in C_{p}(X)$ tal que $f(x)=r_{x}$ para cualquier $x \in A$. De manera que $f \in U \cap C_{p}(X)$ y por lo tanto $C_{p}(X) \cap U \neq \varnothing$ lo cual muestra que $C_{p}(X)$ es denso en $\mathbb{R}^{X}$. Por la Proposición 1.2.2 el número de Souslin de $C_{p}(X)$ es numerable. 
Teorema 1.2.6. [Tk6, Problem 169], [Ar2] Para todo espacio infinito $X$ se tiene que $|X|=\chi\left(C_{p}(X)\right)=w\left(C_{p}(X)\right)$.

Demostración. La familia $\mathcal{B}=\left\{\left[x_{1}, \ldots, x_{n} ; O_{1}, \ldots, O_{n}\right]: n \in \mathbb{N}, x_{i} \in X\right.$ y $O_{i}$ es un intervalo racional para todo $\left.i \leq n\right\}$ es una base de $C_{p}(X)$ (ver [Tk6, Problem 056]) y $|\mathcal{B}| \leq|X|$. Esto muestra que $w\left(C_{p}(X)\right) \leq|X|$.

Denotemos por $u$ a la función que vale cero en todos los puntos de $X$ y tomemos cualquier base local $\mathcal{C}$ en el punto $u$ en el espacio $C_{p}(X)$. Para cualquier $U \in \mathcal{C}$, podemos elegir un conjunto abierto estándar $O(U)$ tal que $u \in O(U) \subset U$. Es claro que la familia $\{O(U): U \in \mathcal{C}\}$ es también una base local en $u$ y por lo tanto podemos suponer, sin perder generalidad, que los elementos de $\mathcal{C}$ son estándares. Dado un conjunto abierto arbitrario $U=\left[x_{1}, \ldots, x_{n} ; O_{1}, \ldots, O_{n}\right] \in \mathcal{C}$, definimos $\operatorname{supp}(U)=\left\{x_{1}, \ldots, x_{n}\right\}$. Consideremos el conjunto $Y=\bigcup\{\operatorname{supp}(U): U \in \mathcal{C}\}$. Si $x \in X \backslash Y$ entonces existe $U=\left[x_{1}, \ldots, x_{n} ; O_{1}, \ldots, O_{n}\right] \in \mathcal{C}$ con $U \subset W=[x ;(-1,1)]$. Sin embargo, $\operatorname{supp}(U)=x_{1}, \ldots, x_{n} \subset Y \subset X \backslash\{x\}$, de modo que existe $f \in C_{p}(X)$ tal que $f\left(x_{i}\right) \in O_{i}$ y $f(x)=1$ ya que $X$ es de Tychonoff. Es claro que $f \in U \backslash W$ lo cual es una contradicción.

En consecuencia, se debe cumplir que $Y=X$ y $|X|=|Y| \leq \chi\left(C_{p}(X)\right)$. Finalmente, las desigualdades $w\left(C_{p}(X)\right) \leq|X| \leq \chi\left(C_{p}(X)\right) \leq w\left(C_{p}(X)\right)$ (ver [Tk6, Problem 156]) muestran que $|X|=\chi\left(C_{p}(X)\right)=w\left(C_{p}(X)\right)$.

Proposición 1.2.7. [Tk6, Problem 172], [Mi] Para todo espacio $X$ se tiene que $n w(X)=n w\left(C_{p}(X)\right)$.

Demostración. Tomemos una red $\mathcal{N}$ del espacio $X$ tal que $|\mathcal{N}|=\kappa=$ $n w(X)$. Para cualquier colección finita $N_{1}, \ldots, N_{k} \in \mathcal{N}$ y cualesquiera intervalos reales $I_{1}, \ldots, I_{k}$ con extremos racionales, definamos $M\left(N_{1}, \ldots, N_{k} ; I_{1}, \ldots, I_{k}\right)=\left\{f \in C_{p}(X): f\left(N_{j}\right) \subset I_{j}\right.$ para todo $\left.j \leq k\right\}$ Es evidente que la familia $\mathcal{M}=\left\{M\left(N_{1}, \ldots, N_{k} ; I_{1}, \ldots, I_{k}\right): k \in \mathbb{N}, N_{1}, \ldots, N_{k} \in\right.$ $\mathcal{N}$ y $I_{j}$ es un intervalo racional para toda $\left.j \leq k\right\}$ tiene cardinalidad $\kappa$. Para probar que $\mathcal{M}$ es una red de $C_{p}(X)$, tomemos cualquier $f \in C_{p}(X)$ y cualquier $U \in \tau\left(f, C_{p}(X)\right)$. Existe un conjunto abierto canónico $V=$ $\left[x_{1}, \ldots, x_{k} ; O_{1}, \ldots, O_{k}\right]$ con $f \in V \subset U$. Sin pérdida de generalidad podemos suponer que los puntos $x_{1}, \ldots, x_{k}$ son distintos. Por la continuidad de la función $f$, podemos encontrar conjuntos abiertos disjuntos $U_{1}, \ldots, U_{k}$ con $x_{i} \in U_{i}$ para cada $i \leq k$ e intervalos racionales $I_{1}, \ldots, I_{k}$ tales que $f\left(U_{j}\right) \subset I_{j} \subset O_{j}$ 
para toda $j \leq k$. Existen $N_{1}, \ldots, N_{k} \in \mathcal{N}$ tales que $x_{j} \in N_{j} \subset U_{j}$ para cada $j \leq k$. Es fácil ver que $f \in M\left(N_{1}, \ldots, N_{k} ; I_{1}, \ldots, I_{k}\right) \subset V \subset$ $U$ lo cual prueba que $\mathcal{M}$ es una red de $C_{p}(X)$. Puesto que $|\mathcal{M}| \leq k=$ $n w(X)$, tenemos que $n w\left(C_{p}(X)\right) \leq n w(X)$ para todo espacio $X$. Por lo tanto, $n w\left(C_{p}\left(C_{p}(X)\right)\right) \leq n w\left(C_{p}(X)\right) \leq n w(X)$ para cualquier espacio $X$. Sin embargo, $X$ se encaja como subespacio cerrado de $C_{p}\left(C_{p}(X)\right)$ lo que implica que $n w(X) \leq n w\left(C_{p}\left(C_{p}(X)\right)\right)$. Las desigualdades así obtenidas muestran que $n w(X)=n w\left(C_{p}(X)\right)$.

Proposición 1.2.8. [Tk6, Problem 173], [GA] Para todo espacio $X$ se tiene que $d(X)=\psi\left(C_{p}(X)\right)=\Delta\left(C_{p}(X)\right)=i w\left(C_{p}(X)\right)$.

Demostración. Supongamos que tenemos un espacio $Y$ y una familia $\gamma \subset$ $\tau(Y \times Y)$ tal que $\Delta_{Y}=\bigcap \gamma$. Fijemos $y \in Y$ y para cualquier $U \in \gamma$ elegimos $O_{U} \in \tau(y, Y)$ tal que $O_{U} \times O_{U} \subset U$. Entonces, $\bigcap\left\{O_{U}: U \in \gamma\right\}=\{y\}$ lo cual prueba que $\psi(Y) \leq \Delta(Y)$.

Ahora supongamos que $f: Z \rightarrow Y$ es una condensación y que $\mathcal{B}$ es una base del espacio $Y$ tal que $|\mathcal{B}|=\kappa=i w(Z)$. Entonces $l\left((Y \times Y) \backslash \Delta_{Y}\right) \leq$ $w\left((Y \times Y) \backslash \Delta_{Y}\right) \leq \kappa$. Para cada $z \in(Y \times Y) \backslash \Delta_{Y}$ podemos elegir $U_{z} \in$ $\tau(z, Y \times Y)$ tal que $\overline{U_{z}} \cap \Delta_{Y}=\varnothing$. La cubierta abierta $\left\{\overline{U_{z}}: z \in(Y \times Y) \backslash \Delta_{Y}\right\}$ del espacio $(Y \times Y) \backslash \Delta_{Y}$ tiene una subcubierta $\mu$ con $|\mu| \leq \kappa$. Por consiguiente, $(Y \times Y) \backslash \Delta_{Y}=\bigcup\{\bar{U}: U \in \mathcal{U}\}$ es igual a la unión de no más que $\kappa$ conjuntos cerrados y en consecuencia $\psi\left(\Delta_{Y}, Y \times Y\right) \leq \kappa$. Puesto que $f$ es una condensación, tenemos que $\psi\left(\Delta_{Z}, Z \times Z\right) \leq \kappa$ de modo que $\Delta(Z) \leq i w(Z)$.

Dado un conjunto estándar $V=\left[x_{1}, \ldots, x_{n} ; O_{1}, \ldots, O_{n}\right] \in \tau\left(C_{p}(X)\right)$, definamos $\operatorname{supp}(V)=\left\{x_{1}, \ldots, x_{n}\right\}$. Tomemos cualquier $\gamma \subset \tau\left(C_{p}(X)\right)$ tal que $\bigcap \gamma=\{u\}$ donde $u \equiv 0 \mathrm{y}|\gamma|=\kappa$. Para cada $U \in \gamma$ existe un conjunto abierto estándar $O_{U}=\left[x_{1}, \ldots, x_{n} ; O_{1}, \ldots O_{n}\right]$ tal que $u \in O_{U} \subset U$. Es fácil verificar que el conjunto $Y=\bigcup\left\{\operatorname{supp}\left(O_{U}\right): U \in \gamma\right\}$ tiene cardinalidad a lo más $\kappa$. Si $X \neq \bar{Y}$, escogemos $x \in X \backslash \bar{Y}$ y $f \in C_{p}(X)$ tal que $f(x)=1$ y $f(Y) \subset\{0\}$. Es inmediato que $f \in \bigcap \gamma \backslash\{u\}$; esta contradicción muestra que el conjunto $Y$ es denso en $X$ y por lo tanto $d(X) \leq|Y| \leq \kappa$. Hemos probado que $d(X) \leq \psi\left(C_{p}(X)\right)$ y aplicando las desigualdades obtenidas previamente, tenemos que $d(X) \leq \psi\left(C_{p}(X)\right) \leq \Delta\left(C_{p}(X)\right) \leq i w\left(C_{p}(X)\right)$. Para concluir la prueba mostraremos que $i w\left(C_{p}(X)\right) \leq d(X)$. Fijemos un conjunto $Y \subset X$ tal que $|Y|=d(X)$ y $X=\bar{Y}$. La proyección $\pi_{y}: C_{p}(X) \rightarrow$ $Z=\pi_{Y}\left(C_{p}(X)\right) \subset C_{p}(Y)$ es una condensación (ver [Tk6, Problem 152]) y 
$w(Z) \leq w\left(C_{p}(Y)\right)=|Y|=d(X)$. Esto muestra que $i w\left(C_{p}(X)\right) \leq d(X)$ lo cual termina la demostración.

Proposición 1.2.9. [Tk6, Problem 174], [No] Para todo espacio $X$ se tiene que $i w(X)=d\left(C_{p}(X)\right)$.

Demostración. Observemos que $i w(X) \leq i w\left(C_{p}\left(C_{p}(X)\right)\right) \leq d\left(C_{p}(X)\right)$. La primera desigualdad se sigue de que $X$ se encaja en $C_{p}\left(C_{p}(X)\right)$ como subespacio cerrado (ver [Tk6, Problem 167]) por lo que podemos aplicar [Tk6, Problem 159(v)]. La segunda desigualdad se sigue de la Proposición 1.2.8.

Por otra parte si $i w(X) \leq \kappa$, tomemos una condensación $f: X \rightarrow Y$ tal que $w(Y) \leq \kappa$. El mapeo $f^{*}$ encaja el espacio $C_{p}(Y)$ en $C_{p}(X)$ como subespacio denso (ver [Tk6, Problem 163(v)]). Llamemos $Z$ al espacio $f^{*}\left(C_{p}(Y)\right)$. Tenemos que $d(Z) \leq n w(Z)=n w\left(C_{p}(Y)\right)=n w(Y) \leq w(Y) \leq \kappa$ y por lo tanto $d\left(C_{p}(X)\right) \leq d(Z) \leq \kappa$ lo que establece que $d\left(C_{p}(X)\right) \leq i w(X)$ y esto concluye la demostración.

Teorema 1.2.10. Arhangel'skii-Pytkeev [Ar2, Theorem II.1.1] Para todo espacio $X$ se tiene que $t\left(C_{p}(X)\right)=\sup \left\{l\left(X^{n}\right): n \in \mathbb{N}\right\}$.

Demostración. Probemos primero que $t\left(C_{p}(X)\right) \leq \kappa=\sup \left\{l\left(X^{n}\right): n \in\right.$ $\mathbb{N}\}$. Denotamos por $u$ a la función tal que $u(x)=0$ para todo $x \in X$. Basta probar que para cualquier $A \in C_{p}(X)$ con $u \in \bar{A}$ existe $B \subset A$ tal que $u \in \bar{B}$ y $|B| \leq \kappa$. Para cualquier $n \in \mathbb{N}$, la familia $\gamma_{n}=\left\{f^{-1}\left(\left(-\frac{1}{n}, \frac{1}{n}\right)\right): f \in A\right\}$ es una $\omega$-cubierta de $X$. Por [Tk6, Problem 148] y [GN] existe una $\omega$-cubierta $\mu \subset$ tal que $|\mu| \leq \kappa$. Por lo tanto existe $B_{n} \subset A$ tal que $\left|B_{n}\right| \leq \kappa$ y la familia $\left\{f^{-1}\left(\left(-\frac{1}{n}, \frac{1}{n}\right)\right): f \in B_{n}\right\}$ es una $\omega$-cubierta de $X$. Sea $B=\bigcup\left\{B_{n}: n \in \mathbb{N}\right\}$. Es claro que $|B| \leq \kappa$, de modo que sólo tenemos que mostrar que $u \in \bar{B}$. Dados $x_{1}, \ldots, x_{k} \in X$ y $\varepsilon>0$, tomemos cualquier $n \in \mathbb{N}$ tal que $\frac{1}{n}<\varepsilon$. Puesto que $\left\{f^{-1}\left(\left(-\frac{1}{n}, \frac{1}{n}\right)\right): f \in B_{n}\right\}$ es una $\omega$-cubierta de $X$, existe $f \in B_{n}$ tal que $\left\{x_{1}, \ldots, x_{k}\right\} \subset f^{-1}\left(\left(-\frac{1}{n}, \frac{1}{n}\right)\right)$. Esto significa que $\left|f\left(x_{i}\right)\right|<\frac{1}{n}<\varepsilon$ para toda $i \leq k$. Por lo tanto $f \in V=O\left(u, x_{1}, \ldots, x_{k}, \varepsilon\right)$ de modo que $V \cap B \neq \varnothing$ para la vecindad arbitraria $V$ de $u$, es decir $u \in \bar{B}$.

Ahora sea $\lambda=t\left(C_{p}(X)\right)$. Debemos probar que $l\left(X^{n}\right) \leq \lambda$ para todo $n \in \mathbb{N}$. Por [Tk6, Problem 148] esto es equivalente a demostrar que cualquier $\omega$-cubierta tiene una $\omega$-subcubierta de cardinalidad a lo más $\lambda$. Sea $\gamma$ una $\omega$-cubierta de $X$ y $A=\left\{f \in C_{p}(X): f^{-1}(\mathbb{R} \backslash\{0\}) \subset U \in \gamma\right\}$. Es fácil ver que 
$w \in \bar{A}$ donde $w(x)=1$ para todo $x \in X$. Puesto que $t\left(C_{p}(X)\right) \leq \lambda$ podemos encontrar $B \subset A$ tal que $w \in \bar{B}$ y $|B| \leq \lambda$. Para cada $f \in B$, tomemos $U_{f} \in \gamma$ para que cual $f^{-1}(\mathbb{R} \backslash 0) \subset U_{f}$. Tenemos que $\mu=\left\{U_{f}: f \in B\right\} \subset \gamma$ y $|\mu| \leq \lambda$. Para verificar que $\mu$ es una $\omega$-cubierta de $X$ tomemos cualquier conjunto finito $F \subset X$. Como $w \in \bar{B}$, existe $f \in B$ tal que $f(x)>0$ para todo $x \in F$. Esto implica que $F \subset f^{-1}(\mathbb{R} \backslash\{0\}) \subset U_{f}$ y en consecuencia $\mu$ es una $\omega$-cubierta de $X$.

En el segundo capítulo del presente texto se demuestra el Corolario 2.2.21 que generaliza el siguiente teorema clásico de Asanov de la $C_{p}$-teoría.

Teorema 1.2.11. Asanov [Ar2, I.4.1] Para todo espacio $X$ y todo $n$ natural se tiene que $t\left(X^{n}\right) \leq l\left(C_{p}(X)\right)$. 


\subsection{Las propiedades de $C_{p}(K)$ cuando $K$ es compacto}

Como hemos visto en la Sección 1.2, la estructura algebraica de los espacios $C_{p}(X)$ implica restricciones fuertes en el comportamiento de los invariantes cardinales de $C_{p}(X)$. En la presente sección veremos que dichas restricciones son aún mayores cuando se estudian espacios de la forma $C_{p}(K)$ para algún espacio compacto $K$. En particular, nos ocuparemos de la clase de los espacios compactos de Eberlein, que se definen como subespacios compactos de $C_{p}(K)$ para algún compacto $K$. Nuestro estudio de las clases de los espacios compactos relevantes en $C_{p}$-teoría incluye también a la clase de los espacios compactos de Gul'ko, es decir, los compactos $K$ para los cuales el espacio $C_{p}(K)$ es Lindelöf $\Sigma$. Adicionalmente, se consideran los espacios compactos de Corson, mismos que son subespacios compactos del $\Sigma$-producto de $\mathbb{R}^{\kappa}$ para algún cardinal $\kappa$.

Proposición 1.3.1. [Tk6 Problem 174],[No] Si K es un espacio compacto entonces $K$ es metrizable si y sólo si $C_{p}(K)$ es separable.

Demostración. Si $K$ es metrizable, entonces $w(K) \leq \omega$ y por lo tanto $i w(K) \leq \omega$. De la Proposición 1.2.9 se sigue que $d\left(C_{p}(K)\right)=\omega$. Si suponemos que $C_{p}(K)$ es un espacio separable entonces, aplicando la Proposición 1.2.9 podemos deducir que existen un espacio segundo numerable $L$ y una condensación $f: K \rightarrow L$. Cualquier condensacion de un compacto es un homeomorfismo y por lo tanto $w(K) \leq \omega$, es decir, $K$ es metrizable.

Proposición 1.3.2. [Ar2, Corollary II.6.19] Si K es un espacio compacto entonces $C_{p}(K)$ es monolítico.

Demostración. Supongamos que $D \subset C_{p}(K)$ es un subcojunto infinito y $X=\bar{D}$. Denotemos por $\varphi$ el producto diagonal de las funciones de $D$ y sea $L=\varphi(K)$. Se sigue de $L \subset \mathbb{R}^{D}$ que $n w(L) \leq w(L) \leq|D|$. La compacidad de $K$ implica que $\varphi$ es $\mathbb{R}$-cociente, de modo que $C_{p}(L)$ es homeomorfo a un subespacio cerrado $Y$ de $C_{p}(K)$ tal que $D \subset Y$ y por consiguiente $X \subset Y$. La relación $n w(X) \leq n w(Y)=n w\left(C_{p}(L)\right)=n w(L) \leq|D|$ termina la demostración. 
Teorema 1.3.3. Dado un espacio compacto $K$, si $Y \subset C_{p}(K)$ separa los puntos de $K$ entonces es posible encontrar un compacto $P$ y un espacio $B$ cerrado en $Y^{\omega} \times \omega^{\omega} \times P$ tal que $C_{p}(X)$ es imagen continua de $B$.

Demostración. Dada una clase $\mathcal{Q}$ de espacios, llamamos $\mathcal{Q}_{\sigma \delta}$ a la clase de todos lo espacios $X$ que se pueden representar como $X=\bigcap\left\{X_{n}: n \in \omega\right\}$ donde cada $X_{n}$ es la unión de una familia numerable de elementos de $\mathcal{Q}$. Para cualquier espacio $X$ definimos el espacio $\mathcal{O}_{\omega}(X)=(X \times \omega)^{\omega}$. Si $X$ es homeomorfo a $\mathcal{O}_{\omega}(X)$ entonces decimos que $X$ es $\omega$-invariante. La clase $\mathcal{P}$ de todos los espacios que son imágenes continuas de un producto del espacio $Y^{\omega} \times \omega^{\omega}$ y algún espacio compacto es $k$-dirigida, ya que $Y^{\omega} \times \omega^{\omega}=\mathcal{O}_{\omega}(Y)$ es $\omega$-invariante. Por [Ar2, Theorem IV.2.4] el que $Y \in \mathcal{P}$ implica que $C_{p}(K) \in$ $(\mathcal{P})_{\sigma \delta}$. Además si $\gamma \in \mathcal{P}$ es numerable entonces $\bigcup \gamma \in \mathcal{P}$. Por lo tanto existe una familia numerable $\left\{Z_{i}: i \in \omega\right\} \subset \mathcal{P}$ tal que $C_{p}(K)=\bigcap\left\{Z_{i}: i \in \omega\right\}$. De modo que es posible encontrar un subespacio cerrado $B^{\prime}$ del producto $T=\Pi\left\{Z_{i}: i \in \omega\right\}$ y un homeomorfismo $\psi: B^{\prime} \rightarrow C_{p}(X)$. Tenemos que $T \in \mathcal{P}$, así que para algún espacio compacto $P$ existe un mapeo continuo sobreyectivo $\varphi: \mathcal{O}_{\omega}(Y) \times P \rightarrow T$, de manera que $B=\varphi^{-1}\left(B^{\prime}\right)$ es cerrado en $\mathcal{O}_{\omega}(Y) \times P$ y $C_{p}(X)=(\psi \circ \varphi)(B)$.

Teorema 1.3.4. Para cualquier clase $\omega$-perfecta $\mathcal{P}$ y cualquier espacio compacto $K$, las siguientes condiciones son equivalentes:

(a) El espacio $C_{p}(K)$ pertenece a la clase $\mathcal{P}$.

(b) Existe un espacio $Y \subset C_{p}(K)$ tal que $Y$ es denso en $C_{p}(K)$ y $Y$ pertenece a la clase $\mathcal{P}$.

(c) Existe un espacio $Y \subset C_{p}(K)$ tal que $Y$ separa los puntos de $K$ y $Y$ pertenece a la clase $\mathcal{P}$.

(d) El espacio $K$ se encaja en $C_{p}(Z)$ para algún espacio $Z$ que pertenece a la clase $\mathcal{P}$.

Demostración. Es claro que $(a) \Longrightarrow(b)$. Para ver que $(b) \Longrightarrow(c)$ basta verificar que si $Y$ es denso en $C_{p}(K)$ entonces $Y$ separa los puntos de $K$. En efecto, si $x$ y $y$ son elementos de $K$ entonces existe $f \in C_{p}(K)$ tal que $f(x)=0$ y $f(y)=1$. De modo que existe $g \in Y \cap O\left(f, x, y, \frac{1}{3}\right)$; es fácil ver que $g(x) \neq g(y)$. 
Para probar que $(c) \Longrightarrow(d)$ hagamos $Z=Y$. Para cada $x \in K$ definimos la función $e_{x}^{Z}: Z \longrightarrow \mathbb{R}$ mediante la fórmula $e_{x}^{Z}(f)=f(x)$ para cada $f \in Z$; observemos que $e_{x}^{Z} \in C_{p}(Z)$. En efecto, dado $x \in K$, la proyección natural $p_{x}: \mathbb{R}^{K} \longrightarrow \mathbb{R}$ sobre el factor indexado por $x$ está definida por $p_{x}(f)=f(x)$ para todo $f \in \mathbb{R}^{K}$. Por lo tanto $e_{x}^{Z}=p_{x} \mid Z$ es un mapeo continuo.

El mapeo de evaluación $E^{Z}: K \longrightarrow C_{p}(Z)$ se define por $E^{Z}(x)=e_{x}^{Z}$ para cada $x \in K$. Para probar que el mapeo $E^{Z}$ es continuo en cada $x \in K$ tomemos cualquier vecindad básica estándar $U=\left(e_{x}^{Z}, f_{1}, \ldots, f_{n}, \varepsilon\right)$ de la función $e_{x}^{Z}$ en $C_{p}(Z)$. El conjunto $V=\bigcap\left\{f_{i}^{-1}\left(\left(f_{i}(x)-\varepsilon, f_{i}(x)+\varepsilon\right)\right): i \leq n\right\}$ es una vecindad abierta de $x$ y es inmediato que $E^{Z}(V) \subset U$. Esto muestra que el mapeo $E^{Z}$ es continuo.

Puesto que $K$ es compacto y una condensación de un compacto es un homeomorfismo, para probar que $E^{Z}(K)$ es homeomorfo a $K$ basta probar que $E^{Z}$ es una inyección. El conjunto $Z$ separa los puntos de $K$ así que para cualesquiera $x, y \in K$ existe $f \in Z$ tal que $e_{x}^{Z}=f(x) \neq f(y)=e_{x}^{Z}(f)$ lo que muestra que las funciones $e_{x}^{Z}$ y $e_{x}^{Z}$ no coinciden, es decir $E^{Z}(x) \neq E^{Z}(y)$. Por lo tanto $E^{Z}$ es inyectiva y encaja a $K$ en $C_{p}(Z)$.

Para probar que $(d) \Rightarrow(a)$, llamemos $X$ al subespacio de $C_{p}(Z)$ que es homeomorfo a $K$. Probaremos que $C_{p}(X)$ pertenece a la clase $\mathcal{P}$. Dado $z \in Z$ definimos $e_{z}^{X}: X \rightarrow \mathbb{R}$ mediante la fórmula $e_{z}^{X}(f)=f(z)$ para cualquier $f \in X$. Recordemos que $e_{z}^{X} \in C_{p}(X)$. Para el mapeo de evaluación $E^{X}: Z \rightarrow C_{p}(X)$ es claro que $E^{X}(Z)$ separa los puntos de $Z$ y $E^{X}(Z) \in \mathcal{P}$. Ahora podemos aplicar el Teorema 1.3.4 para concluir que $C_{p}(X) \in \mathcal{P}$.

Corolario 1.3.5. Si $\mathcal{P}$ es la clase de los espacios $K$-analíticos o Lindelöf $\Sigma$ entonces para cualquier espacio compacto $K$, las siguientes condiciones son equivalentes:

(a) El espacio $C_{p}(K)$ pertenece a la clase $\mathcal{P}$.

(b) Existe un espacio $Y \subset C_{p}(K)$ tal que $Y$ es denso en $C_{p}(K)$ y $Y$ pertenece a la clase $\mathcal{P}$.

(c) Existe un espacio $Y \subset C_{p}(K)$ tal que $Y$ separa los puntos de $K$ y $Y$ pertenece a la clase $\mathcal{P}$.

(d) El espacio $K$ se encaja en $C_{p}(Z)$ para algún espacio $Z$ que pertenece a la $\mathcal{P}$. 
Demostración. Basta observar que las clases de los espacios $K$-analíticos y Lindelöf $\Sigma$ son $\omega$-perfectas.

Teorema 1.3.6. [Ar2, Theorem III.5.8] Si K es un espacio compacto de Eberlein entonces $w(K)=c(K)$.

Demostración. Es fácil ver que la desigualdad $c(K) \leq w(K)$ se cumple para cualquier espacio $K$. Ahora bien, si $K$ es un espacio compacto de Eberlein entonces por [Ar2, Corollary III.5.7] existe un subespacio metrizable $M \subset K$ que es denso en $K$. Aplicamos la Proposición 1.2.4 para obtener la igualdad $c(M)=c(K)$. Es estándar que en los espacios métricos el número de Souslin y densidad coinciden así que $c(M)=d(M)$. Puesto que $M$ es denso en el espacio compacto $K$, es inmediato que $d(K) \leq d(M)=c(M)=c(K)$. La Proposición 1.3.2 implica que $w(K) \leq d(K)=c(K)$. Concluimos que $w(K)=c(K)$.

Teorema 1.3.7. [Ar2, Theorem IV.3.1] Si $K$ es un espacio compacto de Corson entonces $d(K)=w(K)$.

Teorema 1.3.8. [Ar2, theorem IV.8.7] Bajo MA $+\neg \mathbf{C H}$, Si $K$ es un espacio compacto tal que $C_{p}(K)$ es un espacio de Lindelöf entonces $K$ es $\omega$ monolítico. 


\subsection{La propiedad de Lindelöf $\Sigma$ en $C_{p}(X)$.}

La manera más corta de definir la propiedad Lindelöf $\Sigma$ es decir que un espacio $X$ es Lindelöf $\Sigma$ si es imagen continua de un espacio $Y$ que se mapea perfectamente sobre un espacio segundo numerable. Esta definición puede parecer técnica y artificial; sin embargo, es evidente que este concepto es una generalización de la compacidad. Se requiere de cierto esfuerzo para probar que cualquier espacio $\sigma$-compacto y aun cualquier espacio $K$-analítico es Lindelöf $\Sigma$ por lo que uno se podría preguntar por qué esta propiedad se estudia tan intensamente en la Teoría Descriptiva de Conjuntos y el Análisis Funcional. Por ejemplo, los espacios compactos $K$ para los cuales $C_{p}(K)$ es Lindelöf $\Sigma$ aparecen de manera natural en el Análisis Funcional cuando se consideran espacios de Banach débilmente numerablemente determinados. El estudio de esta clase de espacios de Banach es exhaustivo debido a sus buenas propiedades categóricas; una de las caracterizaciones de un espacio de Banach débilmente numerablemente determinado es la propiedad Lindelöf $\Sigma$ del espacio de funciones de la bola unitaria del espacio dual dotado de la topología débil*. Otro indicador de la importancia de una noción topológica es la cantidad de definiciones equivalentes que tiene. Uno puede fácilmente dar diez o más equivalencias de la compacidad, pero si un concepto es de poco interés, entonces es difícil encontrarle siquiera dos condiciones equivalentes. La propiedad Lindelöf $\Sigma$ no es la excepción.

Teorema 1.4.1. Para cualquier espacio $X$ las siguientes condiciones son equivalentes:

1. El espacio $X$ es Lindelöf $\Sigma$.

2. Existe un espacio compacto $K$ y un espacio segundo numerable $M$ tales que $X$ es imagen continua de un subespacio cerrado de $K \times M$.

3. El espacio $X$ pertenece a cualquier clase que contenga a todos los espacios compactos, los espacios segundo numerables y sea invariante bajo subespacios cerrados, productos finitos e imágenes continuas.

4. Existe una multifunción compacto-valuada superiormente semicontinua sobreyectiva $\varphi: M \rightarrow X$ para algún espacio segundo numerable $M$.

5. Existe una multifunción compacto-valuada superiormente semicontinua sobreyectiva $\varphi: P \rightarrow X$ para algún subespacio de los números irracionales $P$. 
6. Existe una cubierta compacta $\mathcal{C}$ del espacio $X$ para la cual es posible encontrar una familia numerable $\mathcal{N}$ que es una red mod $\mathcal{C}$ en el sentido de que para cada $C \in \mathcal{C}$, si $U \in \tau(C, X)$ entonces existe $N \in \mathcal{N}$ tal que $C \subset N \subset U$.

7. Existe una cubierta compacta $\mathcal{C}$ del espacio $X$ para la cual es posible encontrar una familia numerable $\mathcal{Q}$ de subconjuntos cerrados de $X$, que es una red $\bmod \mathcal{C}$.

8. Existe una familia numerable $\mathcal{F}$ de subconjuntos compactos de $\beta X$ tal que $\mathcal{F}$ separa $X$ de $\beta X \backslash X$ en el sentido de que para cada $x \in X y$ $z \in \beta X \backslash X$ existe $F \in \mathcal{F}$ para el cual $x \in F$ y $z \notin F$.

9. Existe una compactificación $b X$ del espacio $X$ y una familia numerable $\mathcal{K}$ de subconjuntos compactos de $b X$ la cual separa $X$ de $b X \backslash X$.

10. Existe un espacio $Y$ tal que $X \subset Y$ y para alguna familia numerable $\mathcal{K}$ de subconjuntos compactos de $Y$ para la cual tenemos que $X \subset \cup \mathcal{K} y$ $\mathcal{K}$ separa $X$ de $Y \backslash X$.

Corolario 1.4.2. Todo espacio $\sigma$-compacto, o de manera más general, todo espacio $K$-analítico tiene la propiedad Lindelöf $\Sigma$.

Demostración. Es inmediato de la condición 5 del Teorema 1.4.1 y de la definición de espacio $K$-analítico.

Corolario 1.4.3. Todo espacio con una red numerable es Lindelöf $\Sigma$.

Demostración. Si un espacio $X$ tiene una red numerable $\mathcal{N}$ entonces $\mathcal{N}$ es una red con respecto a la cubierta compacta $\{\{x\}: x \in X\}$ del espacio $X$. La condición 6 del Teorema 1.4 .1 concluye la demostración.

Corolario 1.4.4. Todo espacio Lindelöf Čech-completo es Lindelöf $\Sigma$.

Demostración. Supongamos que $X$ es un espacio Lindelöf Čech-completo; entonces existe una familia $\mathcal{U}=\left\{U_{n}: n \in \omega\right\}$ de subconjuntos abiertos de $\beta X$ tal que $X=\bigcap \mathcal{U}$. Fijemos $n \in \omega$; para cada $x \in X$ elegimos $V_{x} \in \tau(x, X)$ tal que $c l_{\beta X}\left(V_{x}\right) \subset U_{n}$. Por la propiedad de Lindelöf del espacio $X$ podemos encontrar un conjunto numerable $A_{n} \subset X$ tal que $X \subset \bigcup\left\{V_{x}: x \in A_{n}\right\}$. 
Hagamos $\mathcal{F}_{n}=\left\{\operatorname{cl}_{\beta X}\left(V_{x}\right): x \in A_{n}\right\}$ para cada $n \in \omega$. Es inmediato que $\mathcal{F}=\bigcup_{n \in \omega} \mathcal{F}_{n}$ es una familia numerable de subconjuntos compactos de $\beta X$ que separa $X$ de $\beta X \backslash X$ así que $X$ es Lindelöf $\Sigma$ por la condición 8 del Teorema 1.4.1.

Proposición 1.4.5. Si $p: X \rightarrow Y$ es una multifunción sobreyectiva compactovaluada superiormente semicontinua y $X$ es un espacio Lindelöf $\Sigma$ entonces $Y$ también es Lindelöf $\Sigma$. En consecuencia, cualquier imagen continua de un espacio Lindelöf $\Sigma$ es un espacio Lindelöf $\Sigma$ y cualquier preimagen perfecta de un espacio Lindelöf $\Sigma$ es un espacio Lindelöf $\Sigma$.

Demostración. Es un ejercicio fácil verificar que la composición de dos mapeos compacto-valuados superiormente semicontinuos sobreyectivos es un mapeo compacto-valuado superiormente semicontinuo sobreyectivo de modo que nuestra primera afirmación es consecuencia inmediata de la condición 4 del Teorema 1.4.1. Cualquier mapeo continuo es un mapeo compacto-valuado y superiormente semicontinuo; esto implica que cualquier imagen continua de un espacio Lindelöf $\Sigma$ es un espacio Lindelöf $\Sigma$. Finalmente, si $p: X \rightarrow Y$ es un mapeo perfecto sobreyectivo y el espacio $Y$ es Lindelöf $\Sigma$ entonces haciendo $\varphi(y)=p^{-1}(y)$ para todo $y \in Y$ obtenemos un mapeo compactovaluado superiormente semicontinuo sobreyectivo $\phi: Y \rightarrow X$ de modo que $X$ es Lindelöf $\Sigma$.

Proposición 1.4.6. Si $X$ es un espacio Lindelöf $\Sigma$ y un conjunto $F \subset X$ es cerrado en $X$ entonces $F$ también es Lindelöf $\Sigma$.

Demostración. Aplicamos la condición 6 del Teorema 1.4.1 para tomar una cubierta compacta $\mathcal{C}$ del espacio $X$ tal que existe una red $\mathcal{N}$ con respecto a $\mathcal{C}$. Es inmediato que $\mathcal{C}^{\prime}=\{C \cap F: C \in \mathcal{C}\}$ es una cubierta compacta de $F$ y la familia $\{N \cap F: n \in \mathcal{N}\}$ es una red numerable $\bmod \left(\mathcal{C}^{\prime}\right)$ de manera que $F$ es Lindelöf $\Sigma$.

Proposición 1.4.7. Si $X_{i}$ es un espacio Lindelöf $\Sigma$ para cada $i \in \omega$ entonces $X=\prod_{i \in \omega} X_{i}$ es también un espacio Lindelöf $\Sigma$. 
Demostración. Aplicamos la condición 4 del Teorema 1.4.1 para encontrar un espacio segundo numerable $M_{i}$ y una multifunción compacto-valuada superiormente semicontinua sobreyectiva $\varphi_{i}: M_{i} \rightarrow X_{i}$ para cada $i \in \omega$. El espacio $M=\prod_{i \in \omega} M_{i}$ es segundo numerable; para cualquier $x \in M$ definimos $\varphi(x)=\prod_{i \in \omega} \varphi_{i}(x(i))$. Es estándar verificar que $\phi: M \rightarrow X$ es una multifunción compacto-valuada superiormente semicontinua sobreyectiva y por lo tanto $X$ es un espacio Lindelöf $\Sigma$.

Corolario 1.4.8. Si $Y$ es un espacio y $X_{i} \subset Y$ tiene la propiedad Lindelöf $\Sigma$ para todo $i \in \omega$ entonces el conjunto $X=\bigcap_{i \in \omega} X_{i}$ también tiene la propiedad Lindelöf $\Sigma$.

Demostración. El espacio $P=\prod_{i \in \omega} X_{i}$ tiene la propiedad Lindelöf $\Sigma$ por la Proposición 1.4.7. Es fácil ver que la diagonal $D=\{x \in P: x(i)=$ $x(j)$ para cualesquiera $i, j \in \omega\}$ del producto $P$ es un subespacio cerrado de $P$ homeomorfo a $X$, de manera que $X$ es un espacio Lindelöf $\Sigma$ por la Proposición 1.4.6.

Proposición 1.4.9. Si $X$ es un espacio, $X_{i} \subset X$ tiene la propiedad Lindelöf $\Sigma$ para cada $i \in \omega$ y $X=\bigcup_{i \in \omega} X_{i}$ entonces $X$ también tiene la propiedad Lindelöf $\Sigma$.

Demostración. Aplicamos la condición 6 del Teorema 1.4.1 para encontrar una cubierta compacta $\mathcal{C}_{i}$ del espacio $X_{i}$ tal que existe una red numerable $\mathcal{N}_{i}$ con respecto a $\mathcal{C}_{i}$ para cada $i \in \omega$. Es fácil verificar que $\mathcal{C}=\bigcup_{i \in \omega} \mathcal{C}_{i}$ es una cubierta compacta de $X$ y $\mathcal{N}=\bigcup_{i \in \omega} \mathcal{N}_{i}$ es una red numerable mod $\mathcal{C}$ de manera que $X$ es un espacio Lindelöf $\Sigma$.

Teorema 1.4.10. [Ar2 Theorem II.6.21] Todo espacio Lindelöf $\Sigma$ es estable.

Demostración. Dado que cada imagen continua de un espacio Lindelöf $\Sigma$ es Lindelöf $\Sigma$, basta mostrar que, para todo espacio $X$ que es Lindelöf $\Sigma$, 
si $X$ se condensa sobre un espacio de peso $\kappa$ entonces $n w(X) \leq \kappa$. Fijemos una condensación $\phi: X \rightarrow Y$ tal que $Y$ tiene una base $\mathcal{B}$ con $|\mathcal{B}| \leq \kappa$. Si $\mathcal{F}=\left\{\varphi^{-1}(\bar{B}): B \in \mathcal{B}\right\}$ entonces es fácil ver que la familia $\mathcal{F}$ es $T_{1}$-separadora en $X$, es decir,

(1) para cualesquiera puntos distintos $x, y \in X$ existe $F \in \mathcal{F}$ tal que $x \in F \subset X \backslash\{y\}$.

Elegimos una cubierta compacta $\mathcal{C}$ del espacio $X$ para la cual existe una red numerable $\mathcal{N}$ con respecto a $\mathcal{C}$. La familia $\mathcal{Q}$ de todas las intersecciones finitas de los elementos de $\mathcal{N} \cup \mathcal{F}$ tiene cardinalidad no mayor que $\kappa$; afirmamos que $\mathcal{Q}$ es una red en $X$. En efecto, tomemos un punto $x \in X$ y $U \in \tau(x, X)$; existe $C \in \mathcal{C}$ que contiene a $x$. El conjunto $P=C \backslash U \subset X \backslash\{x\}$ es compacto y para cada $y \in P$ existe $F_{y} \in \mathcal{F}$ tal que $x \in F_{y}$ y $y \notin F_{y}$. Por lo tanto la familia $\left\{F_{y} \cap P: y \in P\right\}$ tiene intersección vacía; por la compacidad de $P$ podemos encontrar un conjunto finito $A \subset P$ tal que $\bigcap\left\{F_{y}: y \in A\right\} \cap P=\varnothing$. El conjunto $Q=\bigcap\left\{F_{y}: y \in A\right\}$ pertenece a $\mathcal{Q}$ y el conjunto cerrado $H=Q \backslash U$ no interseca a $C$. La familia $\mathcal{N}$ es una red con respecto a $\mathcal{C}$, así que existe $N \in \mathcal{N}$ tal que $C \subset N \subset X \backslash H$. Es inmediato que $E=N \cap Q$ pertenece a $\mathcal{Q}$ y $x \in E \subset U$ de modo que $\mathcal{Q}$ es una red en $X$ y por lo tanto $n w(X) \leq \kappa$.

Corolario 1.4.11. Si $\nu X$ es un espacio Lindelöf $\Sigma$ entonces $X$ es $\omega$-estable. Aquí $\nu X$ es realcompactificación de Hewitt del espacio $X$.

Demostración. Sea $f: X \rightarrow Y$ una función continua sobre un espacio $Y$ para el cual existe una condensación $g: Y \rightarrow M$ de $Y$ sobre un espacio segundo numerable $M$. El espacio $Y$ es realcompacto así que existe un mapeo continuo $h: \nu X \rightarrow Y$ con $h \mid X=f$. En particular, $Y$ es un espacio Lindelöf $\Sigma$. El Teorema 1.4.10 garantiza que $Y$ es estable y por lo tanto $n w(Y) \leq \omega$.

En 1979 Gul'ko probó su teorema clásico (ver [Gu]) el cual afirma que si $K$ es un espacio compacto tal que $C_{p}(K)$ es Lindelöf $\Sigma$ entonces $K$ es Corson, es decir, el compacto $K$ se puede encajar en un $\Sigma$-producto de rectas reales. Este teorema colocó a la clase de tales espacios compactos dentro de la jerarquía de los espacios compactos estudiados en el Análisis Funcional: los compactos de Eberlein, los compactos de Talagrand y los compactos de Corson. Por esta razón, a los compactos de esta clase se les llama compactos de Gul'ko.

Tkachuk probó en [Tk6] las siguientes generalizaciones del resultado de Gul'ko.

Teorema 1.4.12. Para todo espacio $X$ si $C_{p}(X)$ es un espacio Lindelöf $\Sigma$ entonces $X$ se condensa dentro de un $\Sigma$-producto de rectas reales. 
Teorema 1.4.13. Si tanto $X$ como $C_{p}(X)$ son espacios Lindelöf $\Sigma$ entonces $C_{p}(X)$ se condensa linealmente dentro de un $\Sigma$-producto de rectas reales.

En los años ochenta, averiguar si la propiedad de Lindelöf $\Sigma$ en todos los espacios iterados de funciones de un espacio $X$ implicaba red numerable en $X$ era un problema abierto de Arhangel'skii. Sipacheva dio una respuesta negativa probando que si $X$ es un compacto de Eberlein, entonces todos los espacios iterados de funciones sobre $X$ son Lindelöf $\Sigma$. Okunev obtuvo la siguiente generalización radical de dicho resultado de Sipacheva.

Teorema 1.4.14. [Ok, theorem 2.12] Si tanto $X$ como $C_{p}(X)$ son espacios Lindelöf $\Sigma$ entonces $C_{p, n}(X)$ es un espacio Lindelöf $\Sigma$ para cada $n \in \mathbb{N}$.

Al poco tiempo de que Okunev demostró el Teorema 1.4.14, se construyeron espacios que mostraban que la propiedad Lindelöf $\Sigma$ de $C_{p}(X)$ no implica que $C_{p}\left(C_{p}(X)\right)$ sea Lindelöf $\Sigma$; análogamente $C_{p}(X)$ puede no ser Lindelöf $\Sigma$ mientras que $C_{p}\left(C_{p}(X)\right)$ tenga la propiedad Lindelöf $\Sigma$. El siguiente resultado de Tkachuk [Tk4] provee la clasificación completa de la propiedad Lindelöf $\Sigma$ en espacios de funciones iterados.

Teorema 1.4.15. Para un espacio arbitrario $X$

(i) Si $C_{p, 2 k+1}(X)$ es un espacio Lindelöf $\Sigma$ para algún $k \in \omega$ entonces $C_{p, 2 n+1}(X)$ es Lindelöf $\Sigma$ para todo $n \in \omega$.

(ii) Si $C_{p, 2 k+2}(X)$ es un espacio Lindelöf $\Sigma$ para algún $k \in \omega$ entonces $C_{p, 2 n+2}(X)$ es Lindelöf $\Sigma$ para todo $n \in \omega$.

Baturov descubrió una propiedad fundamental de los subespacios de $C_{p}(X)$ para el caso cuando $X$ es Lindelöf $\Sigma$. Aunque su teorema, que se incluye a continuación, ya fue generalizado de muchas maneras, sigue siendo uno de los resultados más citados de $C_{p}$-teoría.

Teorema 1.4.16. [Ar2, Theorem III.6.1] Si $X$ es Lindelöf $\Sigma$ entonces ext $(Y)=$ $l(Y)$ para todo $Y \subset C_{p}(X)$.

Demostración. Es evidente que $\operatorname{ext}(Y) \leq l(Y)$ así que basta probar que si $\kappa$ es un cardinal y $l(Y)>\kappa$ entonces $\operatorname{ext}(Y)>\kappa$. Sin perder generalidad podemos suponer que $X$ es un $p$-espacio Lindelöf ya que los espacios Lindelöf $\Sigma$ son imágenes continuas de los $p$-espacios de Lindelöf. El espacio $X$ se mapea de manera perfecta sobre un espacio segundo numerable. 
Supongamos que $l(Y)>\kappa$. Existe una cubierta abierta $\mathcal{U}$ de $Y$ que no contiene una subcubierta de cardinalidad menor o igual que $\kappa$. Podemos suponer que los elementos de $\mathcal{U}$ son de la forma $\left[x_{1}, \ldots, x_{n} ; G_{1}, \ldots, G_{n}\right]$ y cada $G_{i}$ es un intervalo racional para $i=1, \ldots, n$. Definamos $W_{k}(x, G)=$ $\left[x_{1}, \ldots, x_{k} ; G_{1}, \ldots, G_{k}\right]$ donde $x=\left(x_{1}, \ldots, x_{k}\right) \in X^{k}$ y $G=G_{1} \times \ldots \times G_{k}$. La familia $\mathcal{U}$ puede ser representada como $\mathcal{U}=\bigcup\left\{\mathcal{U}_{n}: n \in \omega\right\}$ donde para cada $n \in \mathbb{N}$ existen $k_{n} \in \mathbb{N}$ y $O_{n} \in \tau\left(\mathbb{R}^{k_{n}}\right)$ tales que $O_{n}$ es igual a un producto de intervalos racionales y si $W_{k}(x, G) \in \mathcal{U}_{n}$ entonces $k=k_{n}$ y $G=O_{n}$. Para cada $n \in \mathbb{N}$ sea $A_{n}=\left\{x \in X^{k_{n}}: W_{k_{n}}(x, G) \in \mathcal{U}_{n}\right\}$. Para cada $f \in C_{p}(X)$ y cada $k \in \mathbb{N}$ llamemos $f^{k}$ a la función de $X^{k}$ en $\mathbb{R}^{k}$ que mapea al punto $\left(x_{1}, \ldots, x_{k}\right)$ en el punto $\left(f\left(x_{1}\right), \ldots, f\left(x_{n}\right)\right)$. En esta notación el hecho de que $\mathcal{U}$ cubre a $Y$ puede escribirse como:

(*) Para cada $f \in Y$ existen $n \in \mathbb{N}$ y $x \in A_{n}$ tales que $f^{k_{n}}(x) \in O_{n}$.

El hecho de que ninguna subfamilia de $\mathcal{U}$ de cardinalidad no mayor que $\kappa$ cubre a $Y$ se expresa de la siguiente manera:

$(* *)$ Si $B_{n} \subset A_{n}$ y $\left|B_{n}\right| \leq \kappa$ para cada $n \in \mathbb{N}$, entonces existe $g \in Y$ tal que $g^{k_{n}}\left(\mathcal{V}_{n}\right) \cap O_{n}=\varnothing$ para todo $n \in \mathbb{N}$.

Por recursión transfinita, vamos a construir un conjunto cerrado y discreto $F=\left\{f_{\alpha}: \alpha<\kappa^{+}\right\} \subset Y$.

Elegimos $f_{0} \in Y$ cualquiera y suponemos que dado $\alpha<\kappa^{+}$se han definido $f_{\beta}$ para cada $\beta<\alpha$. Fijemos $n \in \mathbb{N}$ y tomemos un mapeo perfecto $\phi_{k_{n}}$ de $X^{k_{n}}$ sobre algún espacio segundo numerable $M_{n}$.

Para cada colección finita $\beta_{1}, \ldots, \beta_{r}<\alpha$ consideremos el mapeo $f_{\left(\beta_{1}, \ldots, \beta_{r}\right)}^{n}=$ $f_{\beta_{1}}^{k_{n}} \Delta \ldots \Delta f_{\beta_{r}}^{k_{n}} \Delta \phi_{k_{n}}$. El mapeo $f_{\left(\beta_{1}, \ldots, \beta_{r}\right)}^{n}: X^{k_{n}} \rightarrow \mathbb{R}^{k_{n} \cdot r} \times M_{n}$ es un mapeo perfecto de $X^{k_{n}}$ sobre un espacio segundo numerable.

El espacio $\mathbb{R}^{k_{n} \cdot r} \times M_{n}$ es hereditariamente separable, por lo que podemos encontrar un conjunto numerable $S_{\left(\beta_{1}, \ldots, \beta_{r}\right)}^{n} \subset A_{n}$ tal que $f_{\left(\beta_{1}, \ldots, \beta_{r}\right)}^{n}\left(S_{\left(\beta_{1}, \ldots, \beta_{r}\right)}^{n}\right)$ es denso en $f_{\left(\beta_{1}, \ldots, \beta_{r}\right)}^{n}\left(A_{n}\right)$. Sea $B_{n}^{\alpha}=\bigcup\left\{S_{\left(\beta_{1}, \ldots, \beta_{r}\right)}^{n}: \beta_{1}, \ldots, \beta_{n}<\alpha\right\}$. Es claro que $\left|B_{n}^{\alpha}\right| \leq \kappa$, de modo que por la condición (**) existe $f_{\alpha} \in Y$ tal que $f_{\alpha}\left(B_{n}^{\alpha}\right) \cap O_{n}=\varnothing$ para todo $n \in \mathbb{N}$. Esto termina la construcción del conjunto $F=\left\{f_{\alpha}: \alpha<\kappa^{+}\right\}$.

Mostraremos que $F$ es cerrado y discreto en $Y$. Si esto no sucede, entonces existe $g \in Y$ tal que cualquier vecindad de $g$ contiene una infinidad de elementos de $F$. Para algún $n \in \mathbb{N}$ y $\tilde{x} \in X^{k_{n}}$ tenemos que $g \in W_{k_{n}}\left(\tilde{x}, O_{n}\right)$, es decir $g^{k_{n}}(\tilde{x}) \in O_{n}$. La estrechez de $C_{p}(X)$ es numerable, lo que implica que estrechez de $Y$ no excede a $\kappa$. Por lo tanto, existe $\alpha^{\prime}<\kappa^{+}$tal que $g \in \overline{\left\{f_{\alpha}: \alpha<\alpha^{\prime}\right\}}$. Denotemos por $\alpha_{0}$ al mínimo de ta- 
les $\alpha^{\prime}<\kappa^{+}$y definamos $P=\left\{f_{\alpha} \in W_{k_{n}}\left(\tilde{x}, O_{n}\right): \alpha<\alpha_{0}\right\}$. El conjunto $\left(g^{k_{n}}\right)^{-1}\left(O_{n}\right) \cap\left(\bigcap\left\{\left(f^{k_{n}}\right)^{-1}\left(f^{k_{n}}(\tilde{x})\right): f \in P\right\}\right.$ contiene a $\tilde{x}$ por lo que no es vacío. Sea $T=\bigcap\left\{\left(f^{k_{n}}\right)^{-1}\left(f^{k_{n}}(\tilde{x})\right): f \in P\right\} \backslash\left(g^{k_{n}}\right)^{-1}\left(O_{n}\right)$.

Hay dos casos posibles:

Caso 1: El conjunto $T$ es vacío. Sea $\phi_{k_{n}}(\tilde{x})=\tilde{m}$. Recordemos que nuestro mapeo $\phi_{k_{n}}: X^{k_{n}} \rightarrow M_{n}$ es perfecto, de modo que $\phi_{k_{n}}^{-1}(\tilde{m})$ es compacto. Como el conjunto $\phi_{k_{n}}^{-1}\left(O_{n}\right)$ es abierto, existe un conjunto finito $\left.\left\{f_{\beta_{1}}, \ldots, f_{\beta_{r}}\right\} \subset P\right\}$ tal que

$$
\Phi=\left[\left(\bigcap_{1}^{r}\left(f_{\beta_{i}}^{k_{n}}\right)^{-1}\left(f_{\beta_{i}}^{k_{n}}(\tilde{x})\right)\right) \cap \phi_{k_{n}}^{-1}(\tilde{m})\right] \subset\left(g^{k_{n}}\right)^{-1}\left(O_{n}\right) .
$$

El conjunto $\Phi$ es la preimagen del punto $\left(f_{\beta_{1}}^{k_{n}}(\tilde{x}), \ldots, f_{\beta_{r}}^{k_{n}}(\tilde{x}), \tilde{m}\right)$ bajo el mapeo $f_{\left(\beta_{1}, \ldots, \beta_{r}\right)}^{n}$. Observemos que el mapeo $f_{\left(\beta_{1}, \ldots, \beta_{r}\right)}^{n}$ es perfecto, en particular es cerrado y que $\left(g^{k_{n}}\right)^{-1}\left(O_{n}\right)$ es una vecindad del conjunto $\Phi$. Dado que $\tilde{x} \in$ $A_{n} \cap \Phi$ la preimagen completa de un punto del conjunto $f_{\left(\beta_{1}, \ldots, \beta_{r}\right)}^{n}\left(S_{\left(\beta_{1}, \ldots, \beta_{r}\right)}^{n}\right)$ está contenida en $\left(g^{k_{n}}\right)^{-1}\left(O_{n}\right)$ por lo que es posible encontrar $x^{\prime} \in S_{\left(\beta_{1}, \ldots, \beta_{r}\right)}^{n} \cap$ $\left(g^{k_{n}}\right)^{-1}\left(O_{n}\right)$ lo cual muestra que $g \in W_{k_{n}}\left(x^{\prime}, O_{n}\right)$. Sin embargo, por construcción, si $\alpha>\alpha^{*}=\operatorname{máx}\left\{\beta_{1}, \ldots, \beta_{r}\right\}$ entonces $f_{\alpha} \notin W_{k_{n}}\left(x^{\prime}, O_{n}\right)$. Es claro que $\alpha^{*}<\alpha_{0}$ y que $g \in\left\{f_{\alpha}: \alpha<\alpha_{0}\right\}$ implica que $g \in\left\{f_{\alpha}: \alpha<\alpha^{*}\right\}$ lo que contradice la elección de $\alpha_{0}$.

Caso 2: El conjunto $T$ no es vacío. Tomemos un punto $x^{\prime \prime} \in T$. Es inmediato que $g^{k_{n}}\left(x^{\prime \prime}\right) \neq g^{k_{n}}(\tilde{x})$ puesto que $g^{k_{n}}(\tilde{x}) \in O_{n}$. Además $f^{k_{n}}\left(x^{\prime \prime}\right)=f^{k_{n}}(\tilde{x})$ para toda $f \in P$. Esto implica que $g \notin \bar{P}$ lo cual es una contradicción. De modo que el conjunto $F$ es cerrado y discreto en $Y$.

Proposición 1.4.17. Si un espacio $X$ es Lindelöf $\Sigma$ entonces existe un espacio $Z$ Lindelöf $\Sigma$ tal que $C_{p}(X) \subset Z \subset \mathbb{R}^{X}$.

Demostración. Sea $S=\left\{P_{n}: n \in \omega\right\}$ una red numerable con respecto a una cubierta compacta $\gamma$ del espacio $X$. Una función (no necesariamente continua) $f: X \rightarrow \mathbb{R}$ se llama $S$-acotada en un punto $x \in X$ si existe $P_{n} \in S$ tal que $x \in P_{n}$ y $f\left(P_{n}\right)$ es acotado en $\mathbb{R}$. Si una función es $S$-acotada en cada punto de $X$ entonces se llama $S$-acotada.

Cada $f \in C_{p}(X)$ es $S$ acotada. En efecto, para cada $x \in X$ existe $K \in \gamma$ tal que $x \in K$. Como $f$ es continua existe $U \in \tau(K, X)$ tal que $f(U)$ es acotado en $\mathbb{R}$. Por lo tanto existe $P_{n} \in S$ tal que $x \in K \subset P_{n} \subset U$ y $f\left(P_{n}\right)$ es acotado en $\mathbb{R}$. 
Para el espacio $Z$ de todas las funciones $S$-acotadas en $\mathbb{R}^{X}$ tenemos que $C_{p}(X) \subset Z \subset \mathbb{R}^{X}$.

Probemos que $Z$ es Lindelöf $\Sigma$. Sea $\overline{\mathbb{R}}=\mathbb{R} \cup\{-\infty, \infty\}$ la compactificación natural de $\mathbb{R}$ homeomorfa al intervalo $[-1,1]$. Entonces $\overline{\mathbb{R}}^{X}$ es compacto y $C_{p}(X) \subset Z \subset \overline{\mathbb{R}}^{X}$. En particular $\overline{\mathbb{R}}^{X}$ es una compactificación de $Z$. Para cualesquiera $n, k \in \mathbb{N}$ definamos $F_{n, k}=\left\{g \in \overline{\mathbb{R}}^{X}: g\left(P_{n}\right) \subset[-k, k]\right\}$. La familia $\left\{F_{n, k}: n, k \in \mathbb{N}\right\}$ es numerable. Tomemos $f \in Z$ y $g \in \overline{\mathbb{R}}^{X} \backslash Z$ para verificar que existen $n, k \in \mathbb{N}$ tales que $f \in F_{n, k}$ y $g \notin F_{n, k}$. Como $g \notin Z$, la función $g$ no es $S$-acotada en algún $x_{0} \in X$. Por otra parte $f$ es $S$-acotada en $x_{0}$ por lo que existen $n, k \in \mathbb{N}$ tales que $x_{0} \in P_{n_{0}}$ y $f\left(P_{n_{0}}\right) \subset\left[-k_{0}, k_{0}\right]$; esto significa que $f \in F_{n_{0}, k_{0}}$. Pero $g$ no es acotada en $P_{0}$ puesto que no lo es en $x_{0}$, por lo tanto $g \notin F_{n_{0}, k_{0}}$. Aplicando el inciso 9 del Teorema 1.4.1 concluimos que $Z$ es un espacio Lindelöf $\Sigma$.

Teorema 1.4.18. Dado un espacio $X$, si el espacio $C_{p}(X)$ es Lindelöf $\Sigma$ entonces $\nu X$ es Lindelöf $\Sigma$.

Demostración. Por la Proposición 1.4.17 existe un expacio $Z$ con la propiedad Lindelöf $\Sigma$ tal que $C_{p}\left(C_{p}(X)\right) \subset Z \subset \mathbb{R}^{C_{p}(X)}$. El espacio $X$ es canónicamente homeomorfo a un espacio $X^{\prime} \subset C_{p}\left(C_{p}(X)\right)$. Llamemos $Y$ a la cerradura de $X^{\prime}$ en $Z$. El espacio $Y$ es Lindelöf $\Sigma$ y por lo tanto realcompleto. El espacio $X^{\prime}$ es $C$-encajado en $\mathbb{R}^{C_{p}(X)}$ lo cual implica que cualquier $f \in C_{p}\left(X^{\prime}\right)$ se extiende a una función $g \in C_{p}(Y)$, es decir, $Y=\nu X^{\prime}$ y el espacio $\nu X$, que es homeomorfo a $\nu X^{\prime}=Y$, es Lindelöf $\Sigma$.

Teorema 1.4.19. Dado un espacio $X$, si el espacio $C_{p}(X)$ es Lindelöf $\Sigma$ entonces $C_{p}(X)$ es $\omega$-monolítico.

Demostración. Puesto que el espacio $C_{p}(X)$ es Lindelöf $\Sigma$ podemos aplicar el Teorema 1.4.18 para deducir que $\nu X$ es Lindelöf $\Sigma$. Por el Corolario 1.4.11 el espacio $X$ es $\omega$-estable así que $C_{p}(X)$ es $\omega$-monolítico.

Teorema 1.4.20. [Tk4, Theorem 2.15] Si $\omega_{1}$ es un calibre de un espacio $X$, entonces el espacio $C_{p}(X)$ es Lindelöf $\Sigma$ si y sólo si $X$ es cósmico. 


\subsection{Cubiertas conservativas generales y des- composiciones numerables de $C_{p}(X)$}

Esta sección contiene varios teoremas sobre las propiedades de los espacios generales que admiten una cubierta conservativa con subespacios que tienen "buenas" propiedades topológicas. Aquí el resultado más famoso pertenece a Junnila y Potoczny, quienes demostraron que un espacio con una cubierta conservativa compacta tiene que ser metacompacto pero no necesariamente paracompacto. En esta tesis probaremos que la existencia de una cubierta conservativa compacta de $C_{p}(X)$ implica que $X$ es finito y por lo tanto $C_{p}(X)$ es homeomorfo a $\mathbb{R}^{n}$ para algún $n$ natural. Para poner en claro que nuestros resultados generalizan varios teoremas de Tkachuk y Casarrubias Segura sobre las descomposiciones numerables y finitas de $C_{p}(X)$, en esta sección observamos que si un espacio $X$ tiene una cubierta cerrada numerable con subespacios que tienen una propiedad finito aditiva $\mathcal{P}$, entonces $X$ también tiene una cubierta conservativa de subespacios cerrados con la propiedad $\mathcal{P}$. También presentaremos aquí varios resultados de Tkachuk sobre descomposiciones numerables de espacios de funciones así como el teorema de Terada-Yajima cuyo uso es crucial para esta tesis.

El siguiente hecho es clásico, bien conocido y fácil de demostrar.

Proposición 1.5.1. [En, Theorem 1.1.11] Dado un espacio X, si una familia $\mathcal{F} \subset \exp (X)$ es localmente finita entonces es conservativa.

Tratándose de cubiertas conservativas de espacios generales, es evidente que las cubiertas compactas constituyen el caso más importante del tema. Aun cuando los elementos de una cubierta conservativa son finitos, la situación no es tan fácil de clasificar.

Teorema 1.5.2. Si un espacio $X$ es paracompacto y $\sigma$-discreto entonces $X$ tiene una cubierta conservativa con subespacios finitos.

El Teorema 1.5.2 fue demostrado por Telgarsky; poco después, Potoczny mostró que lo recíproco está lejos de ser cierto.

Ejemplo 1.5.3. (Potoczny [Po]) Considérense los conjuntos $W=\omega_{1}$ y $T=$ $\{(\alpha, \lambda) \in W \times W: \alpha<\lambda\}$. Dado cualquier $\alpha \in W$ hagamos $E_{\alpha}=\{\alpha\} \cup$ $\{(\alpha, \lambda): \alpha<\lambda\} \cup\{(\lambda, \alpha): \alpha>\lambda\}$ y $\mathcal{B}_{\alpha}=\left\{E_{\alpha} \backslash K: K\right.$ es un subconjunto finito de $T\}$; además, $\mathcal{B}_{t}=\{t\}$ para todo $t \in T$. Es fácil verificar que las 
familias $\left\{\mathcal{B}_{x}: x \in W \cup T\right\}$ pueden considerarse bases locales de una topología de Tychonoff $\tau$ en el conjunto $W \cup T$. El espacio $X=(W \cup T, \tau)$ no es normal ya que los conjuntos $\omega$ y $\omega_{1} \backslash \omega$ son cerrados en $X$ sin que sea posible separarlos con abiertos disjuntos. Además, la familia $\{\alpha, \lambda,(\alpha, \lambda):(\alpha, \lambda) \in T\}$ es una cubierta conservativa de $X$ con conjuntos finitos y por lo tanto compactos. De modo que la existencia de una cubierta conservativa con conjuntos finitos ni siquiera implica la normalidad de un espacio.

El Ejemplo 1.5.3 fue una fuerte motivación para encontrar cuándo la existencia de una cubierta compacta conservativa implica paracompacidad. El resultado más importante en esta dirección pertenece a Junnila y Potoczny [PJ, Corollary 2].

Teorema 1.5.4. Un espacio $X$ es localmente compacto y paracompacto si y sólo si $X$ tiene una cubierta conservativa compacta $\mathcal{C}$ tal que la familia $\{\operatorname{Int}(C): C \in \mathcal{C}\}$ cubre a $X$.

A continuación se presenta el resultado más famoso de las cubiertas conservativas compactas que pertenece a Potoczny y Junnila. Recordemos que un espacio $X$ es metacompacto si cada cubierta abierta de $X$ tiene un refinamiento abierto punto finito.

Teorema 1.5.5. Si un espacio $X$ admite una cubierta conservativa con subespacios compactos entonces $X$ es metacompacto.

Los resultados citados muestran que es de importancia la clase $\mathcal{P}$ de los espacios que tienen una cubierta conservativa compacta. Resulta que $\mathcal{P}$ tiene buenas propiedades categóricas. Dados espacios $X_{t}, t \in T$ y un punto $a \in \prod\left\{X_{t}: t \in T\right\}$ recordemos que el $\sigma$-producto de los espacios $X_{t}$ con el centro $a$ es el conjunto $\left\{x \in \prod_{t \in T} X_{t}:|\{t \in T: x(t) \neq a\}|<\omega\right\}$ mismo que se denota por $\sigma \prod\left\{X_{t}: t \in T\right\}$.

Teorema 1.5.6. Sea $\mathcal{P}$ la clase de los espacios que tienen una cubierta conservativa compacta.

(a) Si $X$ pertenece a $\mathcal{P}$ entonces todo subespacio cerrado de $X$ pertenece a $\mathcal{P}$.

(b) Si $X$ pertenece a $\mathcal{P}$ entonces toda imagen cerrada continua de $X$ también pertenece a $\mathcal{P}$. 
(c) Si $\left\{X_{t}: t \in T\right\} \subset \mathcal{P}$ entonces el $\sigma$-producto $\sigma \prod\left\{X_{t}: t \in T\right\}$ de los espacios $X_{t}$ también pertenece a $\mathcal{P}$.

El siguiente resultado de Terada y Yajima [TY, Theorem 2.5] se usará de manera importante para analizar cubiertas conservativas de espacios de funciones.

Teorema 1.5.7. Ningún subconjunto no vacío de tipo $G_{\delta}$ de un espacio numerablemente compacto tiene una cubierta conservativa de subconjuntos densos en ninguna parte.

Demostración. Tomemos cualquier espacio numerablemente compacto $Z$ y fijémonos en un conjunto $X=\bigcap_{n \in \omega} G_{n}$ donde $G_{n} \in \tau^{*}(Z)$ para cada $n \in \omega$. Supongamos que $\mathcal{F}$ es una cubierta conservativa de $X$ cuyos elementos son subespacios de $X$ densos en ninguna parte. Podemos asumir que $\mathcal{F}$ es una cubierta cerrada de $X$. Tomemos $F_{0} \in \mathcal{F}$. Puesto que $F_{0} \neq X$, existe un conjunto $U_{0} \in \tau^{*}(X)$ tal que $\overline{U_{0}} \cap F_{0}=\varnothing$ y ${\overline{U_{0}}}^{Z} \subset G_{0}$. Dado que $\mathcal{F}$ cubre a $X$, existe un conjunto $F_{1} \in \mathcal{F}$ tal que $U_{0} \cap F_{1} \neq \varnothing$. Más aún $U_{0} \backslash F_{1} \neq \varnothing$ implica que existe $U_{1} \in \tau^{*}(X)$ tal que $\overline{U_{1}} \subset U_{0}$, y además $\overline{U_{1}} \cap F_{1}=\varnothing \mathrm{y}$ ${\overline{U_{1}}}^{Z} \subset G_{1}$. Continuando de esta manera podemos construir dos sucesiones $\left\{F_{n}: n \in \omega\right\}$ y $\left\{U_{n}: n \in \omega\right\}$ tales que para todo $n \in \omega:$

(a) $F_{n} \in \mathcal{F}$ y $U_{n} \in \tau(X)$;

(b) $\overline{U_{n+1}} \subset U_{n}$ y ${\overline{U_{n}}}^{Z} \subset G_{n}$;

(c) $\overline{U_{n}} \cap F_{n}=\varnothing$ y $U_{n} \cap F_{n+1} \neq \varnothing$.

La propiedad (c) garantiza la existencia de un punto $x_{n} \in U_{n} \cap F_{n+1}$ para todo $n \in \omega$. Puesto que $Z$ es numerablemente compacto, la sucesión $\left\{x_{n}: n \in \omega\right\}$ tiene un punto de acumulación $z \in Z$. De manera que

$$
z \in \bigcap_{n \in \omega} \overline{\left\{x_{k}: k \geq n\right\}} \subset \bigcap_{n \in \omega}{\overline{U_{n}}}^{Z} \subset \bigcap_{n \in \omega} G_{n}=X .
$$

Tenemos que $z \in \bigcap_{n \in \omega} \overline{U_{n}}$. De la condición $(c)$ se sigue que $\bigcup_{n \in \omega} F_{n}$ no interseca a $\bigcap_{n \in \omega} \overline{U_{n}}$ y por lo tanto $z \notin \bigcup_{n \in \omega} F_{n}$. Por otro lado tenemos que $z \in \overline{\left\{x_{n}: n \in \omega\right\}} \subset \overline{\bigcup_{n \in \omega} F_{n}}=\bigcup_{n \in \omega} F_{n}$ lo cual es una contradicción. 
El estudio realizado en esta tesis fue inspirado por los resultados de Tkachuk y Casarrubias Segura sobre descomposiciones numerables de espacios de funciones. La siguiente afirmación muestra que la existencia de cubiertas conservativas con elementos en $\mathcal{P}$ constituye un caso más general que la posibilidad de descomponer un espacio en unión numerable de subconjuntos cerrados con la propiedad $\mathcal{P}$.

Lema 1.5.8. Dado un espacio $X$ y una propiedad finitamente aditiva $\mathcal{P}$ si $X$ tiene una cubierta cerrada numerable de subespacios con la propiedad $\mathcal{P}$ entonces $X$ tiene una cubierta conservativa de subespacios que tienen la propiedad $\mathcal{P}$.

Demostración. Supongamos que $X=\bigcup\left\{C_{n}: n \in \omega\right\}$, donde $C_{n}$ es cerrado en $X$ y tiene la propiedad $\mathcal{P}$ para cada $n \in \omega$. Dado $m \in \omega$, definamos $F_{m}=\bigcup\left\{C_{n}: n \leq m\right\}$. Es inmediato que cada $F_{m}$ es cerrado y tiene la propiedad $\mathcal{P}$ además de que $X=\bigcup\left\{F_{m}: m \in \omega\right\}$. Para verificar que $\mathcal{F}=$ $\left\{F_{m}: m \in \omega\right\}$ es una familia conservativa, tomemos cualquier subfamilia $\mathcal{F}^{\prime} \subset \mathcal{F}$; mostraremos que $\bigcup \mathcal{F}^{\prime}$ es cerrado. Si $\mathcal{F}^{\prime}$ es finita entonces existe $M \in \omega$ tal que $M=\operatorname{máx}\left\{m: F_{m} \in \mathcal{F}^{\prime}\right\}$. Se sigue que $\bigcup \mathcal{F}^{\prime}=F_{M}$. Si $\mathcal{F}^{\prime}$ es infinita entonces para cada $n \in \omega$ existe $m>n$ tal que $F_{m} \in \mathcal{F}^{\prime}$, lo cual implica que $C_{n} \subset F_{m} \in \mathcal{F}^{\prime}$ y por lo tanto $X=\bigcup\left\{C_{n}: n \in \omega\right\} \subset \bigcup \mathcal{F}^{\prime}$. Esto muestra que $\bigcup \mathcal{F}^{\prime}$ es cerrado para cualquier $\mathcal{F}^{\prime} \subset \mathcal{F}$ es decir $\mathcal{F}$ es una cubierta conservativa del espacio $X$ cuyos elementos tienen la propiedad $\mathcal{P}$.

El siguiente lema constituye la herramienta principal para la demostración de la mayor parte de los resultados del resto de esta sección.

Lema 1.5.9. [Tk1, Lemma 1.1] Si $X$ es un espacio tal que $C_{p}(X)=\bigcup\left\{C_{n}\right.$ : $n \in \omega\}$, entonces existen $f \in C_{p}(X), \varepsilon>0$ y $n \in \omega$, tales que el conjunto $\left(C_{n}+f\right) \cap C(X,(-\varepsilon, \varepsilon))$ es denso en $C_{u}(X,(-\varepsilon, \varepsilon))$ y por lo tanto en $C_{p}(X,(-\varepsilon, \varepsilon))$.

Demostración. Definimos $C_{n}^{\prime}=C_{n} \cap C_{p}^{*}(X)$. Entonces $C_{u}^{*}(X)=\bigcup\left\{C_{n}^{\prime}\right.$ : $n \in \omega\}$. Dado que $C_{u}^{*}(X)$ es un espacio métrico completo, se sigue que $C_{n}^{\prime}$ es denso en $B_{\varepsilon}(g)=\left\{h \in C^{*}(X):|h(x)-g(x)|<\varepsilon\right.$ para todo $\left.x \in X\right\}$ para algunos $g \in C^{*}(X), n \in \omega$, y $\varepsilon>0$. Hagamos $f=-g$. Es fácil ver que $B_{\varepsilon}(g)+f$ es uniformemente denso en $C(X,(-\varepsilon, \varepsilon))$. Esto significa que lo mismo sucede para $\left(C_{n}^{\prime}+f\right) \cap C(X,(-\varepsilon, \varepsilon))=\left(C_{n}+f\right) \cap C(X,(-\varepsilon, \varepsilon))$ como se quería demostrar. 
Corolario 1.5.10. [Tk1, Corollary 1.2] Si $X$ es un espacio tal que $C_{p}(X)=$ $\bigcup\left\{C_{n}: n \in \omega\right\}$, entonces:

(a) Existe un espacio $Z \subset C_{p}(X), Z \simeq C_{p}(X)$, tal que $C_{n} \cap Z$ es denso en $Z$ para algún $n \in \omega$;

(b) Algún $C_{n}$ separa los puntos de los subconjuntos cerrados de $X$.

Corolario 1.5.11. [Tk1, Corollary 1.3] Si $X$ es un espacio tal que $C_{p}(X)=$ $\bigcup\left\{C_{n}: n \in \omega\right\}$, donde cada $C_{n}$ es cerrado en $C_{p}(X)$ entonces algún $C_{n}$ contiene una copia homeomorfa de $C_{p}(X)$.

Demostración. La familia $\left\{C_{n}: n \in \omega\right\}$ es una cubierta cerrada del espacio métrico completo $C_{u}(X)$. Por lo tanto existe $n \in \mathbb{N}$ tal que el interior de $C_{n}$ en $C_{u}(X)$ es no vacío. Esto implica que $B(f, r) \subset C_{n}$ para alguna $f \in C_{p}(X)$ y algún $r \in(0,1)$. El espacio $C_{p}\left(X,\left(-\frac{r}{2}, \frac{r}{2}\right)\right)+f$ es homeomorfo a $C_{p}(X)$ y está contenido en $C_{n}$.

Teorema 1.5.12. [Tk1, Theorem 1.4] Supongamos que $\mathcal{P}$ es una propiedad hereditaria. Si $X$ es un espacio tal que $C_{p}(X)=\bigcup\left\{C_{n}: n \in \omega\right\}$, donde cada $C_{n}$ tiene la propiedad $\mathcal{P}$ y es cerrado en $C_{p}(X)$ entonces $C_{p}(X)$ tiene la propiedad $\mathcal{P}$.

Demostración. Basta aplicar el Corolario 1.5.11.

Corolario 1.5.13. Supongamos que $\mathcal{P}$ es alguna de las siguientes propiedades: normalidad, normalidad hereditaria, paracompacidad hereditaria, realcompacidad, radialidad. Si $X$ es un espacio tal que $C_{p}(X)=\bigcup\left\{C_{n}: n \in \omega\right\}$, donde cada $C_{n}$ tiene la propiedad $\mathcal{P}$ y es cerrado en $C_{p}(X)$ entonces $C_{p}(X)$ tiene la propiedad $\mathcal{P}$.

Demostración. La única de las propiedades enlistadas que no es hereditaria es la realcompacidad. Sin embargo, es claro que algún $C_{n}$ contiene un subespacio cerrado homeomorfo a $C_{p}(X,[0,1])$ así que $C_{p}(X,[0,1])$ es realcompacto. Aplicamos [Tk6, Problem 410] para concluir que el espacio $C_{p}(X)$ es realcompacto. 
Teorema 1.5.14. [Tk1, Theorem 1.6] Supongamos que $\kappa$ es un cardinal. Si $X$ es un espacio tal que $C_{p}(X)=\bigcup\left\{C_{n}: n \in \omega\right\}, y h \pi \chi\left(C_{n}\right) \leq \kappa$ para cada $n \in \omega$ entonces $\chi\left(C_{p}(X)\right) \leq \kappa$.

Demostración. De las suposiciones del teorema se sigue que existe un conjunto $Z \subset C_{p}(X)=\bar{Z}$ para el cual $h \pi \chi(Z) \leq \kappa$. Por lo tanto $\chi\left(C_{p}(X)\right)=$ $\pi \chi\left(C_{p}(X)\right)=\pi \chi(Z) \leq \kappa$ como se quería probar.

Corolario 1.5.15. [Tk1, Corollary 1.7] Si $\kappa$ es un cardinal y $C_{p}(X)$ es igual a la unión de una cantidad numerable de subespacios que tienen una propiedad $\mathcal{P} \in\{$ peso $\leq \kappa$, carácter $\leq \kappa, \pi$-peso hereditario $\leq \kappa, \pi$-carácter hereditario $\leq \kappa\}$, entonces $C_{p}(X)$ tiene la propiedad $\mathcal{P}$ y por consiguiente $|X| \leq \kappa$

Teorema 1.5.16. [Tk1, Theorem 1.8] Supongamos que $\kappa$ es un cardinal. Si $X$ es un espacio tal que $C_{p}(X)=\bigcup\left\{C_{n}: n \in \omega\right\}, y \psi\left(C_{n}\right) \leq \kappa$ para cada $n \in \omega$ entonces $\psi\left(C_{p}(X)\right) \leq \kappa$.

Teorema 1.5.17. [Tk1, Theorem 1.11] Supongamos que $\kappa$ es un cardinal. Si $X$ es un espacio tal que $C_{p}(X)=\bigcup\left\{C_{n}: n \in \omega\right\}$, y $t\left(C_{n}\right) \leq \kappa$ para cada $n \in \omega$ entonces $t\left(C_{p}(X)\right) \leq \kappa$.

Teorema 1.5.18. [Tk1, Theorem 1.13] Si $X$ es un espacio tal que $C_{p}(X)=$ $\bigcup\left\{C_{n}: n \in \omega\right\}, y$ cada $C_{n}$ es Čech-completo entonces $C_{p}(X)$ es Čechcompleto, es decir, $X$ es discreto $y$ numerable.

Demostración. Aplicamos el Lema 1.5 .9 para verificar que $C_{p}(X,[0,1])$ contiene un subespacio denso $Y$, mismo que es Cech-completo. El espacio $X$ es discreto por [Tk1, Theorem 1.13]. El espacio $Y$ y consecuentemente $C_{p}(X,[0,1])$ contiene un subconjunto compacto de carácter numerable y por [Tk6, Problem 170] el espacio $X$ es numerable.

El siguiente ejemplo muestra que la numerabilidad del $\pi$-peso es una propiedad que no es numerablemente aditiva en espacios $C_{p}(X)$.

Ejemplo 1.5.19. Supongamos que $K$ es el conjunto perfecto de Cantor, entonces $C_{p}(K)=\bigcup\left\{C_{n}: n \in \omega\right\}$ donde cada $C_{n}$ es cerrado en $C_{p}(X)$ y $\pi w\left(C_{n}\right)=\omega$. 
Demostración. Basta mostrar que existe un conjunto discreto numerable $A \subset C_{p}(K)$ tal que $C_{p}(K,[-1,1]) \subset \bar{A}$. Si probamos esto, entonces al definir $C_{n}=(n+1) \bar{A}$ obtendremos la descomposición numerable de $C_{p}(K)$ que buscamos.

Sea $S$ la familia de todos los conjuntos $\left\{U_{0}, \ldots, U_{k}, r_{0}, \ldots, r_{k}\right\}$, donde $U_{0}, \ldots, U_{k}$ son subconjuntos abierto-cerrados disjuntos no vacíos de $K$ tales que $K \backslash \bigcup_{i \leq k} U_{i} \neq \varnothing$, y $r_{0}, \ldots, r_{k}$ son números racionales en el intervalo $[-1,1]$.

Elijamos una numeración $\left\{\left\{U_{0}^{n}, \ldots, U_{k_{n}}^{n}, r_{0}^{n}, \ldots, r_{k_{n}}^{n}\right\}: n \in \omega\right\}$, de la familia $S$, y consideremos el conjunto $A=\left\{f_{n}: n \in \omega\right\}$, donde $f_{n} \mid U_{i}^{n}=r_{i}^{n}$ para $i=0, \ldots, k_{n}$ y $f_{n} \mid\left(K \backslash \bigcup\left\{U_{i}^{n}: i \leq k_{n}\right\}\right) \equiv n+2$. Es fácil verificar que $C_{p}(K,[-1,1]) \subset \bar{A} \backslash A$. Si $f_{n} \in A$, entonces $f_{n}(x)=n+2$ para algún $x \in K$. Esto muestra que $O\left(f_{n},\{x\}, \frac{1}{2}\right) \cap A=\left\{f_{n}\right\}$, lo cual implica que $A$ es discreto y completa la demostración. 


\section{Capítulo 2}

\section{Cubiertas conservativas de espacios de funciones}

El estudio de los espacios que pueden representarse como la unión de una familia conservativa de subespacios "buenos" se ha realizado inicialmente dentro del contexto de las propiedades de cubrimientos. Potozny y Junnila probaron en [PJ] que un espacio Hausdorff es metacompacto si admite una cubierta conservativa con subespacios compactos. En [Po], Potozny construyó un ejemplo de un espacio que no es normal y tiene una cubierta conservativa compacta. Smith y Telgarsky establecieron en [STe] que la propiedad de tener una cubierta conservativa compacta se preserva por $\sigma$ productos.

En este capítulo presentamos un estudio sistemático de los espacios de funciones $C_{p}(X)$ representables como la unión de una familia conservativa de subespacios con "buenas" propiedades. En la mayoría de los casos las cubiertas conservativas forman un concepto más amplio que las cubiertas cerradas numerables por lo cual los resultados de esta tesis brindan una generalización de varios teoremas de [Tk3]. 


\subsection{Observaciones generales}

En esta sección presentamos unos resultados sencillos en topología general, mismos que nos permitirán obtener una generalización del teorema de Shakhmatov y Tkachuk que afirma que cualquier espacio $X$ tiene que ser finito si $C_{p}(X)$ es $\sigma$-numerablemente compacto.

Cualquier estudio de cubiertas conservativas tiene sentido solamente si las hipótesis que se quieren verificar resultan ciertas para el caso de cubiertas localmente finitas. Mostraremos que las cubiertas localmente finitas de espacios de funciones conservan prácticamente todas las propiedades clásicas.

Proposición 2.1.1. Si $X$ es un espacio con densidad $\kappa$ entonces $X$ tiene una cubierta conservativa de subespacios con cardinalidad $\kappa$.

Demostración. Tomemos un subespacio denso $D$ del espacio $X$ tal que $|D|=\kappa$. Es inmediato que la familia $\{D \cup\{x\}: x \in X\}$ es una cubierta conservativa de $X$ de subespacios con cardinalidad $\kappa$.

Corolario 2.1.2. Todo espacio separable tiene una cubierta conservativa de subespacios numerables.

Proposición 2.1.3. Supongamos que $\mathcal{F}$ es una cubierta conservativa de un espacio $Z$ y que cada elemento de $\mathcal{F}$ es cerrado. Si $X$ es un subespacio de $Z$ y la densidad de $X$ es $\leq \kappa$, entonces existe una subfamilia $\mathcal{F}^{\prime} \subset \mathcal{F}$ de cardinalidad $\leq \kappa$ que cubre a $X$.

Demostración. Tomemos un subespacio $D \subset X$ tal que $D$ es denso en $X$ y $|D| \leq \kappa$. Para cada $d \in D$ existe $F_{d} \in \mathcal{F}$ tal que $d \in \mathcal{F}$. Hagamos $\mathcal{F}^{\prime}=\left\{F_{d}: d \in D\right\}$. Puesto que $\mathcal{F}^{\prime}$ es conservativa y cerrada, tenemos que $\bigcup \mathcal{F}^{\prime}=\overline{\bigcup \mathcal{F}^{\prime}} \supset \bar{D}=X$.

Proposición 2.1.4. Si $X$ es un espacio cuyo número de Souslin es numerable y $\mathcal{F}$ es una cubierta localmente finita de $X$ conformada por subconjuntos cerrados de $X$, entonces existe una subcubierta numerable $\mathcal{F}^{\prime} \subset \mathcal{F}$ del espacio $X$.

Demostración. Puesto que $\mathcal{F}$ es una cubierta localmente finita del espacio $X$, para cada punto $x \in X$ podemos tomar un abierto $V \in \tau(x, X)$ tal que 
$|\{F \in \mathcal{F}: F \cap V \neq \varnothing\}|<\omega$. Se sigue que la familia $\mathcal{V}=\{V \in \tau(X): V$ se cubre por una subfamilia finita de $\mathcal{F}$ \} es no vacía. De modo que podemos tomar una familia celular máxima disjunta $\mathcal{U} \subset \mathcal{V}$. Para cada $U \in \mathcal{U}$ existe una familia finita $\mathcal{F}_{U} \subset \mathcal{F}$ cuya unión contiene a $U$. Notemos que la unión de $\mathcal{U}$ es densa en $X$ ya que $\mathcal{U}$ es máxima. Definamos $\mathcal{F}^{\prime}=\bigcup\left\{\mathcal{F}_{U}: U \in \mathcal{U}\right\}$. El número de Souslin del espacio $X$ es numerable, de modo que la familia $\mathcal{U}$ es numerable y por lo tanto $\mathcal{F}^{\prime}$ lo es también. La familia $\mathcal{F}$ es conservativa por la Observación 1.5.1, por lo cual $X=\overline{\cup \mathcal{U}} \subset \overline{\bigcup_{\mathcal{F}}^{\prime}}=\bigcup \mathcal{F}^{\prime}$.

Proposición 2.1.5. Supongamos que $\mathcal{P}$ es una propiedad que se conserva en subconjuntos de tipo $F_{\sigma}$. Si $C_{p}(X)=\bigcup \mathcal{F}$ y $\mathcal{F}$ es localmente finita $y$ cada $F \in \mathcal{F}$ tiene $\mathcal{P}$ entonces $C_{p}(X)$ es unión finita de subespacios con la propiedad $\mathcal{P}$.

Demostración. Esto se deduce fácilmente del hecho de que $C_{p}(X)$ se encaja como subconjunto de tipo $F_{\sigma}$ en cualquier abierto suyo no vacío.

Corolario 2.1.6. Sea $\mathbb{F}=\{$ realcompacidad, monoliticidad, paracompacidad $\}$. Si una propiedad $\mathcal{P}$ pertenece a la lista $\mathbb{F}$ y $C_{p}(X)$ tiene una cubierta localmente finita cerrada $\mathcal{C}$ tal que cada $C \in \mathcal{C}$ tiene $\mathcal{P}$ entonces $C_{p}(X)$ también tiene la propiedad $\mathcal{P}$.

Demostración. Es consecuencia de la Proposición 2.1.5 y de los Teoremas $2.2,2.5$ y 2.9 de $[\mathrm{Ca}]$.

Corolario 2.1.7. Para cualquier cardinal infinito $\kappa$, consideremos la siguiente lista $\mathbb{F}_{0}=\{\pi$-carácter hereditario $\leq \kappa$, pseudocarácter $\leq \kappa$, estrechez $\leq \kappa\}$. Si una propiedad $\mathcal{P}$ pertenece a $\mathbb{F}_{0}$ y $C_{p}(X)$ tiene una cubierta localmente finita cerrada $\mathcal{F}$ tal que cada $C \in \mathcal{F}$ tiene la propiedad $\mathcal{P}$ entonces $C_{p}(X)$ también tiene la propiedad $\mathcal{P}$.

Demostración. Se sigue de la Proposición 2.1.4 y del Corolario 1.5.10.

Corolario 2.1.8. Consideremos la lista $\mathbb{F}_{1}=\{\check{C}$ ech-completez, la propiedad de Fréchet-Urysohn $\}$. Si una propiedad $\mathcal{P}$ pertenece a la lista $\mathbb{F}_{1}$ y $C_{p}(X)$ tiene una cubierta localmente finita cerrada $\mathcal{F}$ tal que cada $C \in \mathcal{F}$ tiene la propiedad $\mathcal{P}$ entonces $C_{p}(X)$ también tiene $\mathcal{P}$. 
Demostración. Aplíquese la Proposición 2.1.4, el Corolario 1.5.13 y el Teorema 1.12 de [Tk1].

Proposición 2.1.9. Ninguno de los espacios $\mathbb{R}^{\omega}$ y $C_{p}(\mathbb{I})$ admite una cubierta conservativa cerrada de subespacios $\sigma$-pseudocompactos.

Demostración. En [ST, Theorem 3] Shakhmatov y Tkachuk probaron que ninguno de los espacios $\mathbb{R}^{\omega}$ y $C_{p}(\mathbb{I})$ es $\sigma$-pseudocompacto, de manera que basta con observar que se aplica la Proposición 2.1.3 ya que ambos espacios son separables.

Observación 2.1.10. Notemos que la hipótesis de que los elementos de la cubierta conservativa en la Proposición 2.1.9 son cerrados no se puede omitir puesto que $\mathbb{R}^{\omega}$ y $C_{p}(\mathbb{I})$ son ambos separables, y por lo tanto cada uno tiene una cubierta conservativa de subespacios numerables según el Corolario 2.1.2.

Corolario 2.1.11. El espacio $\mathbb{R}^{\omega}$ no es unión conservativa de subespacios cerrados $\sigma$-compactos.

Demostración. Supongamos que $\mathbb{R}^{\omega}$ es igual a la unión de una familia conservativa $\mathcal{F}$ de subespacios cerrados $\sigma$-compactos de $\mathbb{R}^{\omega}$. Como el espacio $\mathbb{R}^{\omega}$ es separable, el Corolario 2.1.3 nos permite concluir que $\mathbb{R}^{\omega}$ es $\sigma$-compacto, lo cual no ocurre. Esta contradicción muestra que el espacio $\mathbb{R}^{\omega}$ no es unión conservativa de subespacios cerrados $\sigma$-compactos.

Lema 2.1.12. Si $C_{p}(X)=\bigcup \mathcal{F}$ y $\mathcal{F}$ es una familia cerrada conservativa de subespacios $\sigma$-pseudocompactos de $C_{p}(X)$, entonces $X$ es pseudocompacto.

Demostración. Si el espacio $X$ no es pseudocompacto, entonces $C_{p}(X)$ contiene un retracto $Y$ homeomorfo a $\mathbb{R}^{\omega}$. Supongamos que $r: C_{p}(X) \rightarrow Y$ es una retracción. El espacio $\mathbb{R}^{\omega}$ es separable por lo que podemos encontrar una familia numerable $\mathcal{F}^{\prime} \subset \mathcal{F}$ tal que $Y \subset \cup \mathcal{F}^{\prime}$. De modo que la igualdad $Y=\bigcup\left\{r(F): F \in \mathcal{F}^{\prime}\right\}$ muestra que $Y=\mathbb{R}^{\omega}$ es $\sigma$-pseudocompacto, lo cual es una contradicción.

Corolario 2.1.13. Si $C_{p}(X)$ es igual a la unión de una familia conservativa de subespacios pseudocompactos, entonces $X$ es pseudocompacto. 
Demostración. Basta notar que la cerradura de un espacio pseudocompacto también es un espacio pseudocompacto de $C_{p}(X)$ y aplicar el Lema 2.1.12.

Nuestro propósito es mostrar que si un espacio de funciones $C_{p}(X)$ tiene una cubierta conservativa de subespacios cerrados $\sigma$-numerablemente compactos entonces $X$ es finito. Utilizaremos los siguientes hechos.

Proposición 2.1.14. Si $C_{p}(X)=\bigcup \mathcal{F}$ y $\mathcal{F}$ es una familia conservativa cerrada de subespacios $\sigma$-numerablemente compactos de $C_{p}(X)$, entonces $f(X)$ es finito para cada $f \in C_{p}(X)$.

Demostración. Tomemos cualquier $f \in C_{p}(X)$ y sea $Y=f(X)$. La igualdad $\omega=w(Y) \geq d\left(C_{p}(Y)\right)$ muestra que $C_{p}(Y)$ es separable. El espacio $X$ es pseudocompacto por el Lema 2.1.12, así que la función $f$ es $\mathbb{R}$-cociente, de modo que el mapeo dual $f^{*}$ encaja a $C_{p}(Y)$ en $C_{p}(X)$ como subespacio cerrado. Por lo tanto $C_{p}(Y)$ tiene una cubierta conservativa de subespacios cerrados $\sigma$ numerablemente compactos y por la Proposición 2.1.3 es $\sigma$-numerablemente compacto. De [Tk1, Theorem 3.11] se sigue que $Y$ es finito.

Proposición 2.1.15. Si $X$ es un espacio de Tychonoff, tal que toda imagen continua de $X$ en $\mathbb{R}$ es finita entonces $X$ es finito.

Demostración. Si $X$ es infinito entonces es posible encontrar un subespacio discreto numerable $D=\left\{x_{n}: n \in \omega\right\} \subset X$ y una familia disjunta de abiertos $\left\{U_{n}: n \in \omega\right\}$ tales que $U_{n} \cap D=\left\{x_{n}\right\}$ para cada $n \in \omega$. Por la propiedad de Tychonoff para cada $n$ existe una $f_{n} \in C(X, \mathbb{I})$ tal que $f_{n}\left(x_{n}\right)=1$ y $f_{n}\left(X \backslash U_{n}\right) \subset\{0\}$. Si hacemos $g_{n}=\frac{1}{(n+1)} f_{n}$ entonces $g=\sum g_{n}$ es continua. En efecto, si $p$ es un punto en algún $U_{n}$ y $V$ es una vecindad de $g(p)$ entonces $g^{-1}(V) \cap U_{n}$ es un abierto que contiene a $p$ y cuya imagen bajo $g$ está contenida en $V$, y por lo tanto $g$ es continua en $p$. Si $p$ no está en la unión de las $U_{n}$ y $\varepsilon>0$ entonces $g(p)=0$ y existe $n \in \omega$ tal que $\frac{1}{(n+1)}<\varepsilon$. La función $h=g_{0}+\ldots+g_{n}$ es continua así que podemos elegir una vecindad $W$ del punto $p$ tal que $h(W) \subset(-\varepsilon, \varepsilon)$. Obsérvese que si $g(x) \neq h(x)$ entonces $x \in U_{k}$ para algún $k>n$ y por lo tanto $g(x)=g_{k}(x) \in\left[0, \frac{1}{k+1}\right] \subset[0, \varepsilon)$. Esto muestra que $g(W) \subset(-\varepsilon, \varepsilon)$, es decir, $g$ es continua en $p$. Tenemos que $\left\{\frac{1}{n+1}: n \in \omega\right\} \subset g(X)$ así que $g(X)$ es infinito, lo cual es una contradicción. 
Corolario 2.1.16. Para todo espacio $X$ las siguientes condiciones son equivalentes:

(a) El espacio $C_{p}(X)$ es igual a la unión de una familia conservativa de subespacios compactos;

(b) El espacio $C_{p}(X)$ es igual a la unión de una familia conservativa cerrada de subespacios $\sigma$-compactos de $C_{p}(X)$;

(c) El espacio $C_{p}(X)$ es igual a la unión de una familia conservativa cerrada de subespacios $\sigma$-numerablemente compactos;

(d) El espacio $X$ es finito.

Demostración. Es evidente que $(a)$ implica $(b)$ y $(b)$ implica $(c)$. Aplicamos la Proposición 2.1.14 y la Proposición 2.1.15 para verificar que $(c)$ implica $(d)$. Si $n \in \omega$ entonces $\mathbb{R}^{n}$ se cubre por una familia numerable creciente de bolas compactas, lo que muestra que $(d)$ implica $(a)$.

Aun si quitamos la hipótesis de que los elementos de una cubierta conservativa de $C_{p}(X)$ son cerrados, tenemos el siguiente resultado:

Corolario 2.1.17. Si para un espacio $X$ el espacio $C_{p}(X)$ admite una cubierta conservativa de subespacios numerablemente compactos entonces $X$ es finito.

Demostración. Supongamos que $\mathcal{C}$ es una cubierta conservativa de $C_{p}(X)$ conformada por subespacios numerablemente compactos de $C_{p}(X)$. Aplicamos el Corolario 2.1.13 para deducir que $X$ es pseudocompacto. De manera que cada $C \in \mathcal{C}$ es compacto por [Ar2, Theorem III.4.23]. La equivalencia $(a) \Longleftrightarrow(d)$ del Corolario 2.1.16 nos permite concluir que $X$ es finito. 


\subsection{Cubiertas conservativas cerradas de $C_{p}(X)$ y $C_{p}(X, \mathbb{I})$}

Puesto que la topología de $C_{u}(X)$ es más fina que la de $C_{p}(X)$, toda cubierta conservativa cerrada de $C_{p}(X)$ es también una cubierta conservativa de $C_{u}(X)$. Es bien conocido el hecho de que $C_{u}(X)$ es un espacio Čechcompleto, por lo cual tiene la propiedad de Baire. En particular, si la familia $\left\{F_{n}: n \in \omega\right\}$ es una cubierta cerrada de $C_{u}(X)$ entonces algún $F_{n}$ tiene interior no vacío en el espacio $C_{u}(X)$. Un resultado más fuerte pertenece a Terada y Yajima, quienes en [TY, Theorem 3.5] establecieron que si $Z$ es un espacio Cech-completo y $\mathcal{F}$ es una cubierta conservativa cerrada de $Z$ entonces algún $F \in \mathcal{F}$ tiene interior no vacío en $Z$. Aprovecharemos este resultado para analizar las cubiertas conservativas de los espacios $C_{p}(X)$.

Proposición 2.2.1. Dado un espacio $X$, una función $f \in C_{p}(X)$, y un $\varepsilon>0$, definimos $I(f, \varepsilon)=\left\{g \in C_{p}(X):|g(x)-f(x)| \leq \varepsilon\right.$ para todo $\left.x \in X\right\}$. Entonces

(a) Si $U \in \tau\left(C_{u}(X)\right)$ entonces para cualquier $f \in U$ existe $\varepsilon>0$ tal que $I(f, \varepsilon) \subset U$.

(b) Para cualesquiera $f \in C_{p}(X)$ y $\varepsilon>0$ existe un homeomorfismo $\varphi: C_{p}(X) \rightarrow C_{p}(X)$ tal que $\varphi\left(C_{p}(X, \mathbb{I})\right)=I(f, \varepsilon)$.

(c) Cada espacio $I(f, \varepsilon)$ es un retracto de $C_{p}(X)$.

(d) Cada espacio $I(f, \varepsilon)$ contiene un subespacio homeomorfo a $C_{p}(X)$.

Demostración. (a) Tomemos $f \in U \in \tau\left(C_{u}(X)\right)$; existe $r>0$ tal que $B(f, r) \subset U$. Elegimos un número $\varepsilon$ tal que $0<\varepsilon<\operatorname{mín}\{1 / 2, r / 2\}$. Si $g \in I(f, \varepsilon)$ entonces $|f(x)-g(x)| \leq \varepsilon<\operatorname{mín}\{1 / 2, r / 2\}$ para cada $x \in X$ lo cual implica que $\sup \{|f(x)-g(x)|: x \in X\} \leq \varepsilon<\operatorname{mín}\{1, r\}$; de modo que $d(f, g)<r$ y por lo tanto $g \in B(f, r)$ e $I(f, \varepsilon) \subset U$.

(b) Para $f \in C_{p}(X)$ y $\varepsilon>0$ definimos $\varphi: C_{p}(X) \rightarrow C_{p}(X)$ por medio de la fórmula $\varphi(g)=2 \varepsilon\left(g-\frac{1}{2}\right)+f$ para cada $g \in C_{p}(X, \mathbb{I})$. Es fácil verificar que la función $\varphi$ es un homeomorfismo tal que $\varphi\left(C_{p}(X, \mathbb{I})\right)=I(f, \varepsilon)$.

(c) Se sigue de (b) que existe un homeomorfismo $\varphi: C_{p}(X) \rightarrow C_{p}(X)$ tal que $\varphi\left(C_{p}(X, \mathbb{I})\right)=I(f, \varepsilon)$. Por lo tanto basta probar que $C_{p}(X, \mathbb{I})$ es un retracto de $C_{p}(X)$. Definamos $\varphi: C_{p}(X) \rightarrow C_{p}(X,(-\infty, 1])$ por medio de la 
fórmula $\varphi(f)(x)=\operatorname{mín}\{f(x), 1\}$ para todo $x \in X$. Asimismo consideremos el mapeo $\psi: C_{p}(X) \rightarrow C_{p}(X,[0,+\infty))$ tal que $\psi(f)(x)=\operatorname{máx}\{f(x), 0\}$ para todo $x \in X$. Es claro que $\varphi \circ \psi: C_{p}(X) \rightarrow C_{p}(X, \mathbb{I})$ es una retracción continua.

(d) Basta con aplicar (b) y notar que $C_{p}(X, \mathbb{I})$ contiene al subespacio $C_{p}(X,(0,1))$ que es homeomorfo a $C_{p}(X)$.

Proposición 2.2.2. Para un espacio arbitrario $X$, si $\mathcal{C}$ es una cubierta conservativa cerrada de $C_{p}(X)$ o de $C_{p}(X, \mathbb{I})$ entonces existe $C \in \mathcal{C}$ tal que $U \subset C$ para algún $U$ abierto no vacío de $C_{u}(X)$.

Demostración. En efecto, $\mathcal{C}$ es una cubierta conservativa cerrada $C_{u}(X)$ o de $C_{u}(X, \mathbb{I})$ así que el teorema de Terada-Yajima (véase 1.5.15) se puede aplicar para concluir que el interior en $C_{u}(X)$ de algún $C \in \mathcal{C}$ es no vacío.

Corolario 2.2.3. Para un espacio arbitrario $X$, si $\mathcal{C}$ es una cubierta conservativa cerrada de $C_{p}(X)$ o de $C_{p}(X, \mathbb{I})$ entonces existe $C \in \mathcal{C}$ tal que $I(f, \varepsilon) \subset \mathcal{C}$ para algún $\varepsilon>0$.

Demostración. Se sigue de la Proposición 2.2.1.

Corolario 2.2.4. Si $X$ es un espacio y $\mathcal{C}$ es una cubierta conservativa cerrada de $C_{p}(X)$ o $C_{p}(X, \mathbb{I})$ entonces algún $C \in \mathcal{C}$ contiene un subespacio homeomorfo a $C_{p}(X)$.

Demostración. Se sigue de la Proposición 2.2.2 y del Corolario 2.2.3.

El siguiente corolario responde afirmativamente al Problema 4.5 de [Gue].

Corolario 2.2.5. Supongamos que $\mathcal{P}$ es una propiedad topológica hereditaria y que $C_{p}(X, \mathbb{I})$ o $C_{p}(X)$ tiene una cubierta conservativa cerrada $\mathcal{C}$ tal que cada $C \in \mathcal{C}$ tiene la propiedad $\mathcal{P}$. Entonces $C_{p}(X)$ también tiene la propiedad $\mathcal{P}$.

Demostración. Por el Corolario 2.2.4, existe $C \in \mathcal{C}$ tal que algún $I \subset C$ es homeomorfo a $C_{p}(X)$; puesto que $C$ tiene la propiedad $\mathcal{P}$, el espacio $I$ y por lo tanto $C_{p}(X)$ tienen la propiedad $\mathcal{P}$.

Resulta que el Problema 4.7 y la segunda parte del Problema 4.6 de [Gue] tienen respuestas positivas como puede verse en el siguiente corolario. 
Corolario 2.2.6. Dado un cardinal infinito $\kappa$ consideremos la siguiente lista de propiedades topológicas:

$\mathbb{L}_{0}=\{$ peso $\leq \kappa$, peso de red $\leq \kappa, i$-peso $\leq \kappa$, carácter $\leq \kappa$, número hereditario de Lindelöf $\leq \kappa$, pseudocarácter $\leq \kappa$, estrechez $\leq \kappa$, spread $\leq \kappa$, número diagonal $\leq \kappa$, densidad hereditaria $\leq \kappa, \kappa$-monoliticidad $\}$.

Si una propiedad $\mathcal{P}$ pertenece a la lista $\mathbb{L}_{0}$ y en $C_{p}(X, \mathbb{I})$ o en $C_{p}(X)$ existe una cubierta conservativa cerrada $\mathcal{C}$ tal que cada $C \in \mathcal{C}$ tiene la propiedad $\mathcal{P}$ entonces $C_{p}(X)$ también tiene $\mathcal{P}$.

El siguiente resultado resuelve el Problema 4.8 de [Gue].

Corolario 2.2.7. Consideremos la siguiente lista de propiedades topológicas: $\mathbb{L}_{1}=\{$ metrizabilidad, propiedad de Fréchet-Urysohn, diagonal pequeña, realcompacidad hereditaria, propiedad de Whyburn, propiedad de ser espacio perfecto, propiedad de ser espacio funcionalmente perfecto $\}$.

Si una propiedad $\mathcal{P}$ pertenece a la lista $\mathbb{L}_{1}$ y en $C_{p}(X, \mathbb{I})$ o en $C_{p}(X)$ existe una cubierta conservativa cerrada $\mathcal{C}$ tal que cada $C \in \mathcal{C}$ tiene la propiedad $\mathcal{P}$ entonces $C_{p}(X)$ también tiene $\mathcal{P}$.

Si una propiedad $\mathcal{P}$ no es hereditaria y $C_{p}(X)$ posee una cubierta conservativa cerrada por subespacios que tienen $\mathcal{P}$ entonces $C_{p}(X)$ no necesariamente tiene la propiedad $\mathcal{P}$.

En efecto, en el Ejemplo 1.5.14 se muestra que si $K$ es el conjunto de Cantor entonces existe una familia numerable $\left\{F_{n}: n \in \omega\right\}$ de subconjuntos cerrados de $C_{p}(K)$ tal que $\bigcup_{n \in \omega} F_{n}=C_{p}(K)$ y cada $F_{n}$ tiene una $\pi$-base numerable, pero $C_{p}(K)$ no tiene una $\pi$-base numerable. Es fácil verificar que la familia $\left\{F_{n}: n \in \omega\right\}$ es conservativa, por lo que el $\pi$-peso numerable no se preserva por medio de uniones conservativas cerradas. Sin embargo, para las propiedades que son hereditarias a subespacios cerrados tenemos el siguiente resultado.

Teorema 2.2.8. Dado un espacio $X$ y una propiedad $\mathcal{P}$ hereditaria con respecto a subespacios cerrados, si en $C_{p}(X, \mathbb{I})$ existe una cubierta conservativa cerrada $\mathcal{C}$ tal que cada $C \in \mathcal{C}$ tiene la propiedad $\mathcal{P}$ entonces $C_{p}(X, \mathbb{I})$ también tiene $\mathcal{P}$.

Demostración. Por el Corolario 2.2.3 existe $C \in \mathcal{C}$ tal que $I(f, \varepsilon) \subset C$ para alguna $f \in C$ y $\varepsilon>0$. La Proposición 2.2.1(b) completa la prueba. 
Corolario 2.2.9. Dado un cardinal infinito $\kappa$ consideremos la siguiente lista de propiedades topológicas:

$\mathbb{L}_{2}=\{$ número de Lindelöf $\leq \kappa$, extent $\leq \kappa$, número de Nagami $\leq \kappa\}$.

Si una propiedad $\mathcal{P}$ pertenece a la lista $\mathbb{L}_{1}$ y en $C_{p}(X, \mathbb{I})$ o en $C_{p}(X)$ existe una cubierta conservativa cerrada $\mathcal{C}$ tal que cada $C \in \mathcal{C}$ tiene $\mathcal{P}$ entonces $C_{p}(X, \mathbb{I})$ también tiene la propiedad $\mathcal{P}$.

Corolario 2.2.10. Consideremos la siguiente lista $\mathbb{L}_{3}$ de propiedades topológicas:

$\mathbb{L}_{3}=\{K$-analiticidad, propiedad Lindelöf $\Sigma$, normalidad, secuencialidad $\}$.

Si una propiedad $\mathcal{P}$ pertenece a la lista $\mathbb{L}_{3}$ y $C_{p}(X, \mathbb{I})$ o $C_{p}(X)$ tiene una cubierta conservativa cerrada $\mathcal{C}$ tal que cada $C \in \mathcal{C}$ tiene $\mathcal{P}$ entonces $C_{p}(X, \mathbb{I})$ también tiene la propiedad $\mathcal{P}$.

Corolario 2.2.11. Si $C_{p}(X, \mathbb{I})$ tiene una cubierta conservativa cerrada $\mathcal{C}$ tal que cada $C \in \mathcal{C}$ es realcompacto entonces $C_{p}(X)$ es realcompacto.

Demostración. Puesto que la realcompacidad es una propiedad hereditaria en subespacios cerrados, podemos aplicar el Teorema 2.2.8 para deducir que $C_{p}(X, \mathbb{I})$ es realcompacto. Claramente el espacio $C_{p}(X)$ es homeomorfo a $C_{p}(X,(0,1)) \subset C_{p}(X, \mathbb{I})$. Es fácil ver que $C_{p}(X,(0,1))$ es el resultado de quitarle a $C_{p}(X, \mathbb{I})$ una unión de subconjuntos de tipo $G_{\delta}$. Se sigue que $C_{p}(X,(0,1))$ y por lo tanto $C_{p}(X)$ es realcompacto.

Corolario 2.2.12. Dado un espacio $X$, si $C_{p}(X)$ tiene una cubierta conservativa cerrada con subespacios $\breve{C}$ ech-completos, entonces $X$ es discreto.

Demostración. Por el Teorema 2.2.8 tenemos que el espacio $C_{p}(X, \mathbb{I})$ es Cech-completo y por lo tanto el espacio $X$ es discreto (ver Teorema 1.13 de $[\mathrm{Tk} 3])$.

Teorema 2.2.13. Dado un espacio $X$, si $C_{p}(X, \mathbb{I})$ tiene una cubierta conservativa cerrada conformada por subespacios $\sigma$-numerablemente compactos, entonces $C_{p}(X, \mathbb{I})$ es numerablemente compacto.

Demostración. Aplicamos el Teorema 2.2.8 para verificar que $C_{p}(X, \mathbb{I})$ es $\sigma$-numerablemente compacto. Por $[\mathrm{Tk} 1,1.5 .3]$ el espacio $C_{p}(X, \mathbb{I})$ es numerablemente compacto.

El siguiente corolario aporta una respuesta positiva al Problema 4.3 de [Gue]. 
Corolario 2.2.14. Si $C_{p}(X, \mathbb{I})$ tiene una cubierta conservativa cerrada $\mathcal{C}$ tal que cada $C \in \mathcal{C}$ es $\sigma$-compacto entonces $X$ es discreto.

Demostración. Puesto que la $\sigma$-compacidad es una propiedad hereditaria a subespacios cerrados, podemos aplicar el Teorema 2.2.8 para concluir que $C_{p}(X, \mathbb{I})$ es $\sigma$-compacto. Por lo tanto $X$ es discreto según [Tk1, 1.5.2].

Teorema 2.2.15. Dado un espacio $X$ y una propiedad $\mathcal{P}$ que es invariante bajo mapeos cocientes, si en $C_{p}(X, \mathbb{I})$ existe una cubierta conservativa cerrada $\mathcal{C}$ tal que cada $C \in \mathcal{C}$ tiene $\mathcal{P}$ entonces el espacio $C_{p}(X, \mathbb{I})$ también tiene la propiedad $\mathcal{P}$.

Demostración. Por el Corolario 2.2.3 existe $C \in \mathcal{C}$ tal que $I(f, \varepsilon) \subset C$ para algún $f \in C$ y $\varepsilon>0$. Aplicamos la Proposición 2.2.1(c) para verificar que $I(f, \varepsilon)$ es un retracto de $C_{p}(X)$. De modo que $I(f, \varepsilon)$ también es un retracto de $C$ lo cual implica que es imagen cociente $C$. La Proposición 2.2.1(b) completa la demostración.

Corolario 2.2.16. Dado un cardinal infinito $\kappa$ consideremos la siguiente lista de propiedades topológicas:

$\mathbb{L}_{4}=\{\kappa$-estabilidad, estrechez funcional débil $\leq \kappa$, estrechez funcional $\leq \kappa\}$ Si una propiedad $\mathcal{P}$ está en la lista $\mathbb{L}_{4}$ y $C_{p}(X, \mathbb{I})$ tiene una cubierta conservativa cerrada $\mathcal{C}$ tal que cada $C \in \mathcal{C}$ tiene $\mathcal{P}$ entonces $C_{p}(X, \mathbb{I})$ también tiene la propiedad $\mathcal{P}$. Si $\mathcal{P}$ es la $\kappa$-estabilidad, entonces $C_{p}(X)$ tiene la propiedad $\mathcal{P}$.

Demostración. La propiedad $\mathcal{P}$ es invariante bajo imágenes cocientes por lo que podemos aplicar el Teorema 2.2.15 para ver que $C_{p}(X, \mathbb{I})$ tiene $\mathcal{P}$. Si $\mathcal{P}$ es $\kappa$-estabilidad, entonces $C_{p}\left(C_{p}(X, \mathbb{I})\right)$ es $\kappa$-monolítico por [Ar2, Theorem II.6.8]. El espacio $X$ también es $\kappa$-monolítico puesto que es homeomorfo a un subespacio de $C_{p}\left(C_{p}(X, \mathbb{I})\right)$, de modo que $C_{p}(X)$ es $\kappa$-estable por [Ar2, Theorem II.6.9].

En lo que resta de esta sección consideramos cubiertas conservativas cerradas de $C_{p}(X)$ tales que todos sus elementos son espacios Lindelöf o Lindelöf $\Sigma$. Se sigue del Teorema 2.2.8 que el espacio $C_{p}(X, \mathbb{I})$ debe tener la propiedad correspondiente. Sin embargo, parece una sospecha razonable que todo el espacio $C_{p}(X)$ deba ser Lindelöf o Lindelöf $\Sigma$ respectivamente. Esto no 
Cubiertas conservativas de espacios de funciones

ha resultado sencillo de verificar ni siquiera para espacios con un solo punto no aislado. Presentaremos algunos resultados positivos en esta dirección; a menudo son generalizaciones de algunos teoremas bien conocidos acerca de las propiedades de un espacio $X$ para el cual $C_{p}(X)$ es Lindelöf o Lindelöf $\Sigma$.

Proposición 2.2.17. Dado un espacio $X$ y un cardinal infinito $\kappa$, supongamos que $C_{p}(X)$ tiene una cubierta conservativa cerrada $\mathcal{C}$ tal que $l(C) \leq \kappa$ para cada $C \in \mathcal{C}$. Entonces cualquier familia discreta de abiertos no vacíos de $X$ tiene cardinalidad a lo más $\kappa$.

Demostración. Supongamos que $X$ contiene una familia discreta de subconjuntos abiertos no vacíos de cardinalidad $\kappa^{+}$. Por la Proposición 1.2.2 existe un conjunto $E \subset C_{p}(X)$ homeomorfo a $\mathbb{R}^{\kappa^{+}}$. El espacio $\mathbb{R}^{\kappa^{+}}$tiene densidad no mayor que $\kappa$ así que $d(E) \leq \kappa$ y podemos encontrar una familia $\mathcal{C}^{\prime} \subset \mathcal{C}$ tal que $\left|\mathcal{C}^{\prime}\right| \leq \kappa$ y $E \subset \cup \mathcal{C}^{\prime}$. Es claro que $l\left(\cup \mathcal{C}^{\prime}\right) \leq \kappa$ y $E$ es cerrado en $\bigcup \mathcal{C}^{\prime}$ por lo que $l(E) \leq \kappa$ y por lo tanto $l\left(\mathbb{R}^{\kappa^{+}}\right) \leq \kappa$, lo cual es una contradicción.

Corolario 2.2.18. Supongamos que $\kappa$ es un cardinal infinito $y X$ es un espacio paracompacto tal que $C_{p}(X)$ tiene una cubierta conservativa cerrada $\mathcal{C}$ con $l(C) \leq \kappa$ para cada $C \in \mathcal{C}$. Entonces $l(X) \leq \kappa$; en particular, si $X$ es metrizable entonces $w(X) \leq \kappa$.

Nuestro siguiente paso es demostrar un resultado positivo para los espacios con un solo punto no aislado y generalizar un teorema de Asanov que afirma que la estrechez de cualquier potencia finita de $X$ es numerable siempre que $C_{p}(X)$ es Lindelöf. De hecho, con la demostración de Asanov se obtiene la misma conclusión aun si sólo se asume que $C_{p}(X, \mathbb{I})$ es Lindelöf. Sin embargo, este hecho no es siquiera mencionado en los trabajos de Asanov ni en los muchos artículos panorámicos que aparecieron posteriormente, así que incluimos aquí la demostración.

Lema 2.2.19. Para cualquier espacio $X$ y cualquier cardinal infinito $\kappa$, si $l\left(C_{p}(X, \mathbb{I})\right) \leq \kappa$, entonces $t\left(X^{n}\right) \leq \kappa$ para cada $n \in \omega$.

Demostración. Tomemos $x=\left(x_{1}, \ldots, x_{n}\right) \in X^{n}$ y $A \subset X^{n}$ con $x \in \bar{A}$. Elegimos $O_{i} \in \tau\left(x_{i}, X\right)$ de manera que $O_{i} \cap O_{j}=\varnothing$ si $x_{i} \neq x_{j}$ y $O_{i}=O_{j}$, si $x_{i}=x_{j}$. El conjunto $O=O_{1} \times \ldots \times O_{n}$ es una vecindad de $x$ y $x \in \overline{A \cap O}$ por 
lo que podemos asumir que $A \subset O$. El conjunto $\Phi=\left\{f \in C_{p}(X, \mathbb{I}): f\left(x_{i}\right)=\right.$ 1 para todo $i \leq n\}$ es cerrado en $C_{p}(X, \mathbb{I})$ y por lo tanto $l(\Phi) \leq \kappa$. Dado $y=\left(y_{1}, \ldots, y_{n}\right) \in A$, definimos $W_{y}=\left\{g \in C_{p}(X, \mathbb{I}): g\left(y_{i}\right)>0\right.$ para todo $i \leq n\}$. Ahora, si $f \in \Phi$ entonces $U_{i}=f^{-1}((0,+\infty)) \in \tau\left(x_{i}, X\right)$ para todo $i \leq n$. Puesto que $x \in \bar{A}$, podemos encontrar un punto $y=\left(y_{1}, \ldots, y_{n}\right) \in$ $A \cap\left(U_{1} \times \ldots \times U_{n}\right)$. Se sigue que $f\left(y_{i}\right)>0$ para cada $i \leq n$ y por eso $f \in W_{y}$. Esto muestra que $\Phi \subset \bigcup\left\{W_{y}: y \in A\right\}$.

Dado que $l(\Phi) \leq \kappa$, existe $B \subset A$ tal que $|B| \leq \kappa$ y $\Phi \subset\left\{W_{y}: y \in B\right\}$. Afirmamos que $x \in \bar{B}$. Si no, entonces $V \cap B=\varnothing$ para algún $V=V_{1} \times \ldots \times V_{n}$ donde $x_{i} \in V_{i} \in \tau\left(O_{i}\right)$ para cada $i \leq n$ y $V_{i}=V_{j}$ si $x_{i}=x_{j}$.

Es fácil ver que hay una función $g \in \Phi$ tal que $g(z)=0$ para cualquier $z \in X \backslash\left(\bigcup\left\{V_{i}: i \leq n\right\}\right)$. Tenemos que $g \in W_{y}$ para algún $y=\left(y_{1}, \ldots, y_{n}\right) \in$ $B$. Fijemos cualquier $i \in\{1, \ldots, n\}$; recordemos que $y \in A \subset O$ y por lo tanto $y_{i} \in O_{i}$. Del hecho de que $g\left(y_{i}\right)>0$ se sigue que $y_{i} \in \bigcup\left\{V_{k}: k \leq n\right\}$, así que podemos encontrar $j \leq n$ tal que $y_{i} \in V_{j}$. Recordemos que $V_{j} \subset O_{j}$, de modo que podemos concluir que $y_{i} \in O_{i} \cap O_{j}$ por lo que $O_{i} \cap O_{j} \neq \varnothing$ lo cual muestra que $O_{i}=O_{j}$ y por eso $x_{i}=x_{j}$ por la elección de $O$; esto implica que $V_{i}=V_{j}$. Por lo tanto $y_{i} \in V_{i}$ para cada $i \leq n$; en consecuencia, $y \in\left(V_{1} \times \ldots \times V_{n}\right) \cap B$ lo cual es una contradicción.

El siguiente corolario generaliza el mencionado resultado de Asanov.

Corolario 2.2.20. Dado un espacio $X$, si $C_{p}(X)$ admite una cubierta conservativa cerrada $\mathcal{C}$ tal que $l(C) \leq \kappa$ para cada $C \in \mathcal{C}$, entonces $t\left(X^{n}\right) \leq \kappa$ para todo $n \in \mathbb{N}$.

Es un hecho conocido (ver, por ejemplo, [Le]) que para un espacio $X$ con un solo punto no aislado, el espacio $C_{p}(X)$ es Lindelöf si y sólo si $X$ es Lindelöf y $t\left(X^{n}\right) \leq \omega$ para cualquier $n \in \mathbb{N}$. Nuestra técnica nos permite generalizar este resultado de la siguiente manera.

Corolario 2.2.21. Para un espacio $X$ con un único punto no aislado las siguientes condiciones son equivalentes:

(a) $C_{p}(X)$ es Lindelöf;

(b) $C_{p}(X)$ tiene una cubierta conservativa cerrada compuesta de subespacios de Lindelöf;

(c) El espacio $X$ es Lindelöf $y t\left(X^{n}\right) \leq \omega$ para toda $n \in \mathbb{N}$. 
Demostración. La implicación $(a) \Longrightarrow(b)$ es trivial y $(c) \Longrightarrow(a)$ está demostrada en [Le]. Para verificar que $(b) \Longrightarrow(c)$ observemos primero que $C_{p}(X, \mathbb{I})$ es Lindelöf según el Corolario 2.2.9. De manera que podemos aplicar el Lema 2.2.19 para deducir que $t\left(X^{n}\right) \leq \kappa$ para cada $n \in \omega$. Llamemos $a$ al único punto no aislado del espacio $X$. Si $X \backslash U$ es no numerable para algún $U \in \tau(a, X)$ entonces la familia $\{\{x\}: x \in X \backslash U\}$ es discreta y consiste de abiertos no vacíos de $X$, lo que contradice la Proposición 2.2.17. Por lo tanto $|X \backslash U| \leq \omega$ para cualquier $U \in \tau(a, X)$ así que el espacio $X$ es Lindelöf, es decir, hemos probado que $(b) \Longrightarrow(c)$.

En lo que resta de esta sección consideraremos el caso en que $C_{p}(X)$ tiene una cubierta conservativa cerrada compuesta por subespacios Lindelöf $\Sigma$. En [Gue, Problem 4.11] se preguntó si $C_{p}(X)$ debe ser Lindelöf $\Sigma$ en este caso. Conjeturamos que la respuesta debe ser afirmativa aunque no lo hemos podido probar hasta ahora ni siquiera para el caso de un espacio con un único punto no aislado. A continuación presentamos algunos resultados que confirman nuestra conjetura y generalizan algunos teoremas bien conocidos sobre las propiedades de los espacios $X$ tales que $C_{p}(X)$ es Lindelöf $\Sigma$.

Proposición 2.2.22. Si $X$ es un espacio Lindelöf $\Sigma$ y $C_{p}(X)$ tiene una cubierta conservativa cerrada por subespacios Lindelöf $\Sigma$ entonces $C_{p}(X)$ es un espacio Lindelöf $\Sigma$.

Demostración. Del Corolario 2.2.10 se sigue que $C_{p}(X, \mathbb{I})$ tiene que ser un espacio Lindelöf $\Sigma$. Por lo tanto $C_{p}(X)$ es Lindelöf $\Sigma$ de acuerdo con [Ar2, Proposition IV.9.17].

Un resultado no trivial de Arhangel'skii (ver [Ar2, Theorem IV.9.8]) dice que si $C_{p}(X)$ es un espacio Lindelöf $\Sigma$ entonces es $\omega$-monolítico. Usando nuestra técnica para trabajar con cubiertas conservativas, lo generalizamos de la siguiente manera.

Teorema 2.2.23. Dado un espacio $X$ si $C_{p}(X)$ tiene una cubierta conservativa cerrada conformada por subespacios Lindelöf $\Sigma$ entonces $C_{p}(X)$ es $\omega$-monolítico.

Demostración. Supongamos que $\mathcal{F}$ es una familia conservativa cerrada de subespacios Lindelöf $\Sigma$ de $C_{p}(X)$ tal que $C_{p}(X)=\bigcup \mathcal{F}$. Tomemos cualquier conjunto numerable $A \subset C_{p}(X)$ y definamos el mapeo $\varphi: X \rightarrow C_{p}(A)$ mediante la fórmula $\varphi(x)(f)=f(x)$ para cada $f \in A$; hagamos $Y=\varphi(X)$. 
Observemos que $w(Y) \leq w\left(C_{p}(A)\right) \leq \omega$. No es difícil comprobar que $A \subset$ $\varphi^{*}\left(C_{p}(Y)\right)$.

Existe un espacio $Z$ y mapeos continuos $\varphi^{\prime}: X \rightarrow Z$ y $\xi: Z \rightarrow Y$ tales que $\varphi^{\prime}$ es $\mathbb{R}$-cociente, $\xi$ es inyectiva y $\varphi=\xi \circ \varphi^{\prime}$. El conjunto $E=\left(\varphi^{\prime}\right)^{*}\left(C_{p}(Z)\right)$ es cerrado en $C_{p}(X)$ y $A \subset \varphi^{*}\left(C_{p}(Y)\right) \subset E$ lo que muestra que $\bar{A} \subset E$. El mapeo $\xi$ es una condensación de $Z$ sobre el espacio segundo numerable $Y$; en consecuencia, $d\left(C_{p}(Z)\right)=i w(Z) \leq w(Y) \leq \omega$; elegimos un conjunto numerable $Q \subset E$ tal que $E=\bar{Q}$. Para cada $a \in Q$ elegimos un conjunto $F_{a} \in \mathcal{F}$ tal que $a \in F_{a}$; la familia $\mathcal{G}=\left\{F_{a}: a \in Q\right\}$ es numerable así que el conjunto $G=\bigcup \mathcal{G}$ es un espacio Lindelöf $\Sigma$. Puesto que $G$ es cerrado en $C_{p}(X)$, tenemos que $E=\bar{Q} \subset \overline{\cup \mathcal{G}}=\bigcup \mathcal{G}=G$ por lo que $E$ es un espacio Lindelöf $\Sigma$. Como $E$ es homeomorfo a $C_{p}(Z)$ podemos aplicar [Ar1, Theorem IV.9.8] para ver que $C_{p}(Z)$ es $\omega$-monolítico y por lo tanto $E$ es $\omega$-monolítico también. En consecuencia, $\bar{A}=c l_{E}(A)$ es un espacio cósmico.

Como respuesta a una pregunta de Arhangel'skii, Tkachuk demostró en [Tk4, Theorem 2.15] que si $C_{p}(X)$ es un espacio Lindelöf $\Sigma$ y $\omega_{1}$ es un calibre de $X$, entonces el espacio $X$ es cósmico. Resulta que la propiedad de Lindelöf $\Sigma$ en este resultado se puede sustituir por la existencia de una cubierta conservativa cerrada por subespacios Lindelöf $\Sigma$.

Corolario 2.2.24. Si $\omega_{1}$ es un calibre de un espacio $X$ y $C_{p}(X)$ tiene una cubierta conservativa cerrada conformada por subespacios Lindelöf $\Sigma$, entonces $X$ es cósmico.

Demostración. Fijemos una cubierta conservativa cerrada $\mathcal{L}$ del espacio $C_{p}(X)$ compuesta por subespacios Lindelöf $\Sigma$. Puesto que $\omega_{1}$ es un calibre de $X$, el espacio $C_{p}(X)$ tiene diagonal pequeña de acuerdo con [Tk2, Theorem 1] y también cada elemento de $\mathcal{L}$ tiene diagonal pequeña. De modo que si $L \in \mathcal{L}$ y $K$ es un subespacio compacto de $L$ entonces $K$ tiene diagonal pequeña. Por este motivo, el espacio $K$ no puede contener $\omega_{1}$-sucesiones libres convergentes y así tenemos que $t(K) \leq \omega$ por [JuS, Theorem 1.2] y [Ju, 3.12]. Aplicamos el Teorema 2.2.23 para verificar que $K$ es $\omega$-monolítico; es un procedimiento estándar verificar que un espacio compacto $\omega$-monolítico con estrechez numerable y diagonal pequeña tiene que ser metrizable. Por lo anterior, tenemos que $L \in \mathcal{L}$ tiene diagonal pequeña y una cubierta compuesta de compactos metrizables para la cual existe una red relativa numerable, así que el Theorem 2.1 de [Gr] nos permite concluir que los elementos de $\mathcal{L}$ son cósmicos. Del Corolario 2.2.6 se sigue que $C_{p}(X)$ y $X$ son cósmicos. 
Es un teorema de Arhangel'skii [Ar3, Theorem 10] el hecho de que para cualquier espacio $X$ tal que $C_{p}(X)$ es Lindelöf $\Sigma$, si $s\left(C_{p}(X)\right) \leq \omega$ entonces $X$ es cósmico. Este teorema también puede generalizarse en el mismo espíritu que el anterior.

Corolario 2.2.25. Supongamos que $X$ es un espacio y que $C_{p}(X)$ tiene una cubierta conservativa cerrada de subespacios Lindelöf $\Sigma$. Si además el spread de $C_{p}(X)$ es numerable, entonces $X$ es cósmico.

Demostración. Notemos primero que $s(X \times X) \leq s\left(C_{p}(X)\right) \leq \omega$. Por el Corolario 2.2.10, el espacio $C_{p}(X, \mathbb{I})$ es Lindelöf $\Sigma$; puesto que $X$ se encaja en $C_{p}\left(C_{p}(X, \mathbb{I})\right)$ debe ser monolítico. Esto muestra que $X \times X$ también es monolítico; dado que cada espacio $\omega$-monolítico de spread numerable es hereditariamente Lindelöf (ver [Ar1, Theorem 1.2.9]) podemos concluir que $h l(X \times X) \leq \omega$.

Por lo tanto la diagonal de $X$ es un conjunto $G_{\delta}$; todo espacio Lindelöf con diagonal $G_{\delta}$ tiene $i$-peso numerable según [Ar1, Theorem 2.1.8] de modo que $d\left(C_{p}(X)\right)=i w(X) \leq \omega$. Por el Teorema 2.2.23, el espacio $C_{p}(X)$ es $\omega$-monolítico y por lo tanto $n w(X)=n w\left(C_{p}(X)\right) \leq \omega$. 


\subsection{Cubiertas conservativas generales de $C_{p}(X)$ y $C_{p}(X, \mathbb{I})$}

Para casi cualquier propiedad topológica $\mathcal{P}$, si en un espacio $Z$ existe un subespacio denso $Y$ que tiene $\mathcal{P}$ entonces $Z$ es igual a la unión de una familia conservativa de subespacios con la propiedad $\mathcal{P}$. Para verificar esto, basta considerar la familia $\mathcal{F}=\{Y \cup\{x\}: x \in Z\}$; dado que todos los elementos de $\mathcal{F}$ son densos en $Z$, la familia $\mathcal{F}$ es conservativa. Resulta que si $Z=C_{p}(X)$ para algún espacio $X$ entonces lo recíproco es cierto también.

Teorema 2.3.1. Dado un espacio $X$ y una propiedad topológica $\mathcal{P}$ invariante bajo imágenes continuas, si $C_{p}(X)$ o $C_{p}(X, \mathbb{I})$ admite una cubierta conservativa $\mathcal{C}$ (no necesariamente cerrada) tal que cada $C \in \mathcal{C}$ tiene $\mathcal{P}$ entonces $C_{p}(X, \mathbb{I})$ contiene un subespacio denso con la propiedad $\mathcal{P}$.

Demostración. La familia $\mathcal{C}^{\prime}=\{\bar{C}: C \in \mathcal{C}\}$ es una cubierta conservativa de $C_{p}(X, \mathbb{I})$ (o $C_{p}(X)$ respectivamente). Aplicamos el Corolario 2.2.3 para encontrar una función $f \in C_{p}(X, \mathbb{I})$ y $\varepsilon>0$ tales que $I(f, \varepsilon) \subset C^{\prime}$ para algún $C^{\prime} \in \mathcal{C}^{\prime}$. Por la Proposición 2.2.1, el conjunto $R=I(f, \varepsilon)$ es un retracto de $C_{p}(X)$ homeomorfo a $C_{p}(X, \mathbb{I})$. En consecuencia, existe una retracción $r: C^{\prime} \rightarrow R$; tomemos $C \in \mathcal{C}$ tal que $C^{\prime}=\bar{C}$. El conjunto $r(C)$ es denso en $r\left(C^{\prime}\right)=R$ y tiene la propiedad $\mathcal{P}$. Dado que el espacio $R$ es homeomorfo a $C_{p}(X, \mathbb{I})$, este último también tiene un subespacio denso con la propiedad $\mathcal{P}$.

Observemos que si $\mathcal{C}$ es una cubierta conservativa (no necesariamente cerrada) de $C_{p}(X)$ tal que cada $C \in \mathcal{C}$ tiene una propiedad $\mathcal{P}$, no es evidente que exista una cubierta conservativa de $C_{p}(X, \mathbb{I})$ con subespacios que tengan la propiedad $\mathcal{P}$.

Teorema 2.3.2. Supongamos que $X$ es un espacio y $\mathcal{P}$ es una propiedad $\sigma$ aditiva tal que todos los conjuntos unipuntuales la tienen y es invariante bajo imágenes continuas. Entonces, las siguientes condiciones son equivalentes:

(a) Existe una cubierta conservativa $\mathcal{C}$ de $C_{p}(X)$ tal que cada $C \in \mathcal{C}$ tiene $\mathcal{P}$

(b) Existe una cubierta conservativa $\mathcal{C}$ de $C_{p}(X, \mathbb{I})$ tal que cada $C \in \mathcal{C}$ tiene $\mathcal{P}$ 
(c) $C_{p}(X)$ tiene un subespacio denso con la propiedad $\mathcal{P}$;

(d) $C_{p}(X, \mathbb{I})$ tiene un subespacio denso con la propiedad $\mathcal{P}$.

Demostración. Puesto que $C_{p}(X, \mathbb{I})$ es imagen continua del espacio $C_{p}(X)$, es inmediato que $(c) \Longrightarrow(d)$. Si $D$ es un subespacio denso de $C_{p}(X,[-1,1]) \simeq$ $C_{p}(X, \mathbb{I})$ con la propiedad $\mathcal{P}$ entonces $D_{n}=n \cdot D$ es un subespacio denso de $C_{p}(X,[-n, n])$ con la propiedad $\mathcal{P}$ para cualquier $n \in \mathbb{N}$. Por la $\sigma$-aditividad de $\mathcal{P}$, el conjunto $\bigcup\left\{D_{n}: n \in \mathbb{N}\right\}$ tiene la propiedad $\mathcal{P}$; y como es denso en $C_{p}(X)$, esto demuestra que $(d) \Longrightarrow(c)$ y por lo tanto $(c)$ y $(d)$ son equivalentes. Del Teorema 2.3.1 se sigue que tanto $(a)$ como $(b)$ implican (d). Al inicio de esta sección observamos que $(c) \Longrightarrow(a)$ y $(d) \Longrightarrow(b)$ así que todas las condiciones $(a)-(d)$ son equivalentes.

Corolario 2.3.3. Para cualquier cardinal infinito $\kappa$ consideremos la siguiente lista de propiedades topológicas:

$\mathbb{M}_{0}=\{$ peso de red $\leq \kappa$, spread $\leq \kappa$, número de Lindelöf $\leq \kappa$, densidad hereditaria $\leq \kappa\}$.

Si una propiedad $\mathcal{P}$ pertenece a la lista $\mathbb{M}_{0}$ entonces las siguientes condiciones son equivalentes:

(a) $C_{p}(X)$ tiene una cubierta conservativa $\mathcal{C}$ tal que cada $C \in \mathcal{C}$ tiene $\mathcal{P}$;

(b) $C_{p}(X, \mathbb{I})$ tiene una cubierta conservativa $\mathcal{C}$ tal que cada $C \in \mathcal{C}$ tiene $\mathcal{P}$;

(c) $C_{p}(X)$ tiene un subespacio denso con la propiedad $\mathcal{P}$;

(d) $C_{p}(X, \mathbb{I})$ tiene un subespacio denso con la propiedad $\mathcal{P}$.

Demostración. Observemos que cada propiedad $\mathcal{P}$ en la lista $\mathbb{M}_{0}$ es $\sigma$ aditiva, invariante bajo imágenes continuas y los conjuntos unipuntuales tienen $\mathcal{P}$; el Teorema 2.3.2 termina la demostración.

La siguiente afirmación responde afirmativamente al Problema 4.4 de [Gue].

Corolario 2.3.4. Para cualquier cardinal infinito $\kappa$ consideremos la siguiente lista de propiedades topológicas:

$\mathbb{M}_{1}=\{$ densidad $\leq \kappa$, celularidad punto-finita $\leq \kappa$, calibre $\kappa, \kappa$-separabilidad $\}$. Si una propiedad $\mathcal{P}$ pertenece a la lista $\mathbb{M}_{1}$ entonces las siguientes condiciones son equivalentes: 
(a) $C_{p}(X)$ tiene una cubierta conservativa $\mathcal{C}$ tal que cada $C \in \mathcal{C}$ tiene la propiedad $\mathcal{P}$

(b) $C_{p}(X, \mathbb{I})$ tiene una cubierta conservativa $\mathcal{C}$ tal que cada $C \in \mathcal{C}$ tiene la propiedad $\mathcal{P}$;

(c) $C_{p}(X)$ tiene la propiedad $\mathcal{P}$;

(d) $C_{p}(X, \mathbb{I})$ tiene la propiedad $\mathcal{P}$.

Demostración. Observemos que cada propiedad $\mathcal{P}$ en la lista $\mathbb{M}_{1}$ es $\sigma$ aditiva e invariante bajo imágenes continuas; además, todos los unipuntuales tienen $\mathcal{P}$ y si un espacio $Z$ contiene un subespacio denso con la propiedad $\mathcal{P}$ entonces $Z$ tiene $\mathcal{P}$. Por el Teorema 2.3.2, las condiciones $(a)$ y $(b)$ son equivalentes y se tienen las implicaciones $(c) \Longrightarrow(a)$ y $(d) \Longrightarrow(b)$.

El Teorema 2.3.2 muestra que tanto $(a)$ como $(b)$ implican que $C_{p}(X, \mathbb{I})$ y $C_{p}(X)$ tienen, cada uno, un subespacio denso con la propiedad $\mathcal{P}$. Por la observación anterior, tanto $C_{p}(X)$ como $C_{p}(X, \mathbb{I})$ tienen la propiedad $\mathcal{P}$ así que todas las condiciones $(a)-(d)$ son equivalentes.

El siguiente resultado resuelve positivamente [Gue, Problem 4.1].

Corolario 2.3.5. Para todo espacio $X$, las siguientes condiciones son equivalentes:

(a) $C_{p}(X)$ tiene una cubierta conservativa de subespacios pseudocompactos;

(b) $C_{p}(X)$ tiene una cubierta conservativa cerrada de subespacios pseudocompactos;

(c) $C_{p}(X)$ es $\sigma$-pseudocompacto.

Demostración. Puesto que la cerradura de un conjunto pseudocompacto es pseudocompacta, es inmediato que las condiciones $(a)$ y $(b)$ son equivalentes. Si $C_{p}(X)=\bigcup\left\{C_{n}: n \in \omega\right\}$ y cada $C_{n}$ es pseudocompacto entonces consideremos el conjunto $D_{n}=\bar{C}_{0} \cup \ldots \cup \bar{C}_{n}$ para cada $n \in \omega$. Es sencillo ver que $\left\{D_{n}: n \in \omega\right\}$ es una cubierta conservativa cerrada de $C_{p}(X)$ cuyos elementos son pseudocompactos, de modo que $(c) \Longrightarrow(b)$. Si $C_{p}(X)$ tiene una cubierta conservativa de subespacios pseudocompactos entonces aplicamos el Teorema 2.3.1 para ver que $C_{p}(X, \mathbb{I})$ y por lo tanto $C_{p}(X,[-1,1])$ contiene un subespacio pesudocompacto denso y por lo tanto es pseudocompacto. Dado 
que $X$ es necesariamente pseudocompacto según el Corolario 2.1.13, tenemos que $C_{p}(X)=\bigcup\left\{n \cdot C_{p}(X,[-1,1]): n \in \omega\right\}$ lo cual muestra que $C_{p}(X)$ es $\sigma$-pseudocompacto.

El siguiente enunciado muestra que la respuesta a Problem 4.2 de [Gue] es afirmativa.

Corolario 2.3.6. Para cualquier espacio $X$, las siguientes condiciones son equivalentes:

(a) $C_{p}(X, I)$ tiene una cubierta conservativa de subespacios pseudocompactos;

(b) $C_{p}(X, \mathbb{I})$ tiene una cubierta conservativa cerrada de subespacios pseudocompactos;

(c) $C_{p}(X, \mathbb{I})$ es pseudocompacto.

Demostración. La implicación $(c) \Longrightarrow(b)$ es trivial; como la cerradura de un pseudocompacto es pseudocompacta, es inmediato que las condiciones $(a)$ y $(b)$ son equivalentes. Finalmente, si ocurre $(a)$ entonces el Teorema 2.3.1 muestra que $C_{p}(X, \mathbb{I})$ contiene un subespacio pseudocompacto denso, así que debe ser pseudocompacto.

Corolario 2.3.7. Para cualquier espacio $X$ las siguientes condiciones son equivalentes:

(a) $C_{p}(X, \mathbb{I})$ tiene una cubierta conservativa de subespacios numerablemente compactos;

(b) $C_{p}(X, \mathbb{I})$ contiene un subespacio denso numerablemente compacto.

Demostración. Basta observar que la compacidad numerable se conserva bajo imágenes continuas y aplicar el Teorema 2.3.1.

El siguiente ejemplo muestra que no es posible sustituir $C_{p}(X)$ por $C_{p}(X, \mathbb{I})$ en el enunciado de Corolario 2.1.17. Asimismo este ejemplo implica que no es posible eliminar la hipótesis de que los elementos de la cubierta conservativa en el Teorema 2.2.13 son cerrados.

Ejemplo 2.3.8. Existe un espacio $X$ tal que $C_{p}(X, \mathbb{I})$ contiene un subespacio denso numerablemente compacto pero $C_{p}(X, \mathbb{I})$ no es numerablemente compacto. 
Demostración. Por [Tk6, S.480 Fact 2] existe un espacio $X$ tal que

(a) $X$ se condensa sobre un $P$-espacio.

(b) $X$ se condensa sobre $\mathbb{R}$ y cualquier subconjunto abierto de $X$ tiene cardinalidad $\mathfrak{c}$, en particular, $X$ no tiene puntos aislados.

Mostremos que el espacio $X$ es el que buscamos. Por (a) existe un $P$-espacio $Y$ para el cual es posible encontrar una condensación $r: X \rightarrow Y$. De [Tk6, Problem 397] se sigue que $C_{p}(Y, \mathbb{I})$ es numerablemente compacto. La imagen de $C_{p}(Y, \mathbb{I})$ bajo el mapeo dual $r^{*}: C_{p}(Y) \rightarrow C_{p}(X)$ es un subespacio denso en $C_{p}(X, \mathbb{I})$. Además, por [Tk6, Problem 133] tenemos que $\left.D=r^{*}\left(C_{p}(Y, \mathbb{I})\right)\right)$ es un subespacio numerablemente compacto de $C_{p}(X, \mathbb{I})$.

Para verificar que $C_{p}(X, \mathbb{I})$ no es un espacio numerablemente compacto, basta comprobar que $X$ no es un $P$-espacio por [Tk6, Problem 397]. En efecto, por (b) existe una condensación $t: X \rightarrow \mathbb{R}$, de manera que el conjunto $\{x\}=t^{-1}(t(x))$ es de tipo $G_{\delta}$ para cada $x \in X$. Si $X$ fuese un $P$-espacio, entonces todos sus puntos serían aislados, lo cual no ocurre según la condición (b).

Corolario 2.3.9. Existe un espacio $X$ para el cual $C_{p}(X, \mathbb{I})=\bigcup \mathcal{F}$ donde $\mathcal{F}$ es una familia conservativa y cada $F \in \mathcal{F}$ es numerablemente compacto, pero $C_{p}(X, \mathbb{I})$ no es numerablemente compacto.

Demostración. El Ejemplo 2.3.8 es un espacio $X$ para el cual el espacio $C_{p}(X, \mathbb{I})$ no es numerablemente compacto y contiene un subespacio denso numerablemente compacto $F$. La familia $\left\{F \cup\{f\}: f \in C_{p}(X, \mathbb{I})\right\}$ es una cubierta conservativa de $C_{p}(X, \mathbb{I})$ cuyos elementos son subespacios numerablemente compactos de $C_{p}(X, \mathbb{I})$. 


\subsection{Cubiertas conservativas de $C_{p}(K)$ para un espacio compacto $K$}

El estudio de los espacios $C_{p}(K)$ cuando $K$ es compacto merece un tratamiento particular. En esta sección realizaremos dicho estudio por medio del análisis de las cubiertas conservativas que admiten los mencionados espacios de funciones sobre espacios compactos.

Teorema 2.4.1. Si $K$ es un espacio compacto tal que $w(K)>\kappa$ entonces $C_{p}(K)$ no tiene una cubierta conservativa compuesta por subespacios de cardinalidad $\kappa$.

Demostración. Supongamos que $\mathcal{F}=\left\{F_{\alpha}: \alpha \in I\right\}$ es una cubierta conservativa de $C_{p}(K)$ conformada por subespacios de cardinalidad $\kappa$. Es inmediato que los elementos de $\mathcal{F}$ tienen densidad no mayor a $\kappa$. De modo que podemos aplicar el Corolario 2.3.4 para concluir que $d\left(C_{p}(K)\right) \leq \kappa$ y por lo tanto $w(K) \leq d\left(C_{p}(K) \leq \kappa\right.$, lo cual es una contradicción.

Corolario 2.4.2. Para un espacio compacto $K$, si $C_{p}(K)$ admite una cubierta conservativa conformada por subespacios de cardinalidad menor o igual que c entonces $C_{p}(K)$ tiene cardinalidad a lo más $\mathfrak{c}$.

Demostración. Supongamos que $\mathcal{C}$ es una cubierta conservativa de $C_{p}(K)$ con subespacios de cardinalidad menor o igual que $\mathfrak{c}$. La familia $\mathcal{F}=\{\bar{C}: C \in$ $\mathcal{C}\}$ es una cubierta conservativa de $C_{p}(K)$ con subespacios cerrados. Por [Ar2, Lemma IV.11.3] tenemos que $|F| \leq \mathfrak{c}$ para todo $F \in \mathcal{F}$. Del Teorema 2.4.1 se sigue que $w(X) \leq \mathfrak{c}$ por lo que $d\left(C_{p}(K)\right) \leq \mathfrak{c}$. Apliquemos la Proposición 2.1.3 para ver que la cubierta $\mathcal{F}$ tiene una subcubierta $\mathcal{G}$ de cardinalidad no mayor que $\mathfrak{c}$ por lo que $\left|C_{p}(K)\right| \leq \mathfrak{c}$.

Al inicio del capítulo notamos que es útil saber si un espacio de funciones $C_{p}(X)$ es separable, para así poder reducir una cubierta conservativa cerrada a una numerable. Para el caso de los espacios compactos, daremos una caracterización en términos de cubiertas conservativas.

Corolario 2.4.3. Para todo espacio compacto infinito $K$ las siguientes condiciones son equivalentes:

(a) Existe una cubierta conservativa $\mathcal{F}$ de $C_{p}(K)$ tal que los elementos de $\mathcal{F}$ son cósmicos. 
(b) Existe una cubierta conservativa $\mathcal{F}$ de $C_{p}(K)$ tal que los elementos de $\mathcal{F}$ son separables.

(c) Existe una cubierta conservativa $\mathcal{F}$ de $C_{p}(K)$ tal que los elementos de $\mathcal{F}$ son numerables.

(d) El espacio $K$ es metrizable.

Demostración. Es inmediato que $(a)$ implica $(b)$, y que $(c)$ implica $(b)$. Ahora, si $K$ es metrizable, entonces $C_{p}(K)$ es separable, de modo que la igualdad $d\left(C_{p}(K)\right)=i w(K)=w(K)=n w(K)=n w\left(C_{p}(K)\right)$ muestra que $(d)$ implica $(a)$. Para verificar que $(d)$ implica $(c)$, tomemos un subespacio numerable $D$ denso en $C_{p}(K)$ y para cada $f \in C_{p}(K)$ definamos el conjunto $D_{f}=D \cup\{f\}$. Es claro que $\mathcal{D}=\left\{D_{f}: f \in C_{p}(X)\right\}$ es una cubierta conservativa de $C_{p}(K)$ cuyos elementos son numerables. De modo que basta probar que $(b)$ implica $(d)$. Supongamos que $K$ no es metrizable; esto implica que $w(X)>\omega$ puesto que $K$ es compacto. Consecuentemente, $d\left(C_{p}(K)\right)>\omega$ así que por el Corolario 2.3.4 podemos concluir que la condición (b) no se cumple.

Notemos que en el Corolario 2.4.3 no se considera el caso en que para un espacio compacto $K$ el espacio $C_{p}(K)$ admite una cubierta conservativa cerrada de subespacios segundo numerables. El motivo es que en este caso particular se puede decir aún más sobre el compacto $K$ como se muestra a continuación.

Corolario 2.4.4. Si $K$ es un espacio compacto infinito, entonces $C_{p}(K)$ admite una cubierta conservativa cerrada con subespacios segundo numerables si y sólo si $K$ es numerable.

Demostración. Si $K$ es numerable, entonces $C_{p}(K)$ es segundo numerable. Si $C_{p}(K)$ tiene una cubierta conservativa cerrada con subespacios segundo numerables, entonces por el corolario 2.4.3 el espacio $K$ es metrizable, lo que significa que $C_{p}(K)$ es separable. Por lo tanto podemos aplicar la Proposición 2.1.3 para ver que $C_{p}(K)$ tiene una cubierta cerrada numerable con subespacios segundo numerables, así que podemos aplicar [Tk3, Corollary 1.7] para concluir que $|X| \leq \omega$.

Aún no es claro si es posible prescindir de la hipótesis de que los elementos de la cubierta conservativa en el Corolario 2.4.4 sean cerrados. 
Corolario 2.4.5. Si $K$ es un espacio compacto entonces las siguientes condiciones son equivalentes:

(a) $C_{p}(K)$ tiene una cubierta conservativa de subespacios $\sigma$-compactos.

(b) $C_{p}(K, \mathbb{I})$ tiene una cubierta conservativa de subespacios $\sigma$-compactos.

(c) K es un espacio compacto de Eberlein.

Demostración. Primero observamos que $K$ es un compacto de Eberlein si y sólo si $C_{p}(X)$ (o equivalentemente, $C_{p}(X, \mathbb{I})$ ) contiene un subespacio denso $\sigma$-compacto. Ahora aplicamos el Teorema 2.3.1.

En [Ar2, IV.2] Arhangel'skii definió las clases $\omega$-perfectas. A continuación veremos que estas propiedades resultan relevantes para el tema que abordamos.

Proposición 2.4.6. Si $\mathcal{P}$ es una clase $\omega$-perfecta y $K$ es un espacio compacto entonces las siguientes condiciones son equivalentes:

(a) $C_{p}(K)$ tiene una cubierta conservativa de subespacios que pertenecen a la clase $\mathcal{P}$.

(b) $C_{p}(K, \mathbb{I})$ tiene una cubierta conservativa de subespacios que pertenecen a la clase $\mathcal{P}$.

(c) $C_{p}(K)$ pertenece a la clase $\mathcal{P}$.

Demostración. Las implicaciones $(c) \Longrightarrow(a)$ y $(c) \Longrightarrow(b)$ son triviales. Si ocurre $(a)$ u ocurre $(b)$ entonces podemos aplicar el Teorema 2.3.1 para convencernos de que $C_{p}(K, \mathbb{I})$ contiene un subespacio denso $Z$ que pertenece a la clase $\mathcal{P}$. Por lo tanto $Z$ separa los puntos de $K$ así que podemos aplicar [Ar2, Proposition IV.3.3] para deducir que $C_{p}(K)$ pertenece a la clase $\mathcal{P}$.

Corolario 2.4.7. Si $X$ es un espacio compacto y $\mathcal{P}$ es la $K$-analiticidad o la propiedad de ser Lindelöf $\Sigma$, entonces las siguientes condiciones son equivalentes:

(a) $C_{p}(X)$ tiene una cubierta conservativa de subespacios con la propiedad $\mathcal{P}$. 
(b) $C_{p}(X, \mathbb{I})$ tiene una cubierta conservativa de subespacios con la propiedad $\mathcal{P}$.

(c) $C_{p}(X)$ tiene la propiedad $\mathcal{P}$.

Demostración. Observemos que tanto la $K$-analiticidad como la propiedad de ser Lindelöf $\Sigma$ son $\omega$-perfectas, por lo que podemos aplicar la Proposición 2.4.6.

En otras palabras, el Corolario 2.4.7 muestra que un espacio compacto $K$ es un compacto de Gulk'o si y sólo si $C_{p}(K)$ tiene una cubierta conservativa de subespacios Lindelöf $\Sigma$. Asimismo este corolario prueba que un espacio compacto $K$ es un compacto de Talagrand si y sólo si $C_{p}(K)$ tiene una cubierta conservativa de subespacios $K$-analíticos. De manera que las cubiertas conservativas de $C_{p}(K)$ nos permiten caracterizar a tres de las clases de espacios compactos que se estudian en el Análisis Funcional: los espacios compactos de Eberlein, los de Talagrand y los comapctos de Gulk'o.

El Corolario 2.4.7 se cumple debido a que para todo compacto $K$, si $C_{p}(K)$ contiene un subespacio denso Lindelöf $\Sigma$, entonces todo el espacio $C_{p}(X)$ es Lindelöf $\Sigma$. Este es un hecho bien conocido que sugiere la siguiente pregunta de manera natural. ¿Es posible encontrar alguna extensión $\mathcal{C}$ de la clase de los espacios compactos tal que para cada espacio $X$ de la clase $\mathcal{C}$ si $C_{p}(X)$ contiene un subespacio denso Lindelöf $\Sigma$ entonces $C_{p}(X)$ mismo es Lindelöf $\Sigma$ ? El siguiente ejemplo (construido por Okunev [Ok, Example 2.7] para otros fines) muestra que no es posible extender la propiedad en cuestión ni siquiera a la clase de los espacios $\sigma$-compactos.

Ejemplo 2.4.8. Existe un espacio $\sigma$-compacto $X$ tal que $C_{p}(X)$ no es Lindelöf pero contiene un subconjunto $\sigma$-compacto $Q$ que es denso $C_{p}(X)$.

Demostración. Para construir el ejemplo que buscamos, consideremos el $\sigma$-producto $S=\left\{x \in\{0,1\}^{\omega_{1}}:\left|x^{-1}(1)\right|<\omega\right\}$ en el espacio $\{0,1\}^{\omega_{1}}$ y hagamos $a(\alpha)=1$ para cualquier $\alpha<\omega_{1}$. El espacio $X=S \cup\{a\}$ es el prometido. Observemos primero que $S$ es $\sigma$-compacto de modo que $X$ mismo es $\sigma$-compacto. Para cada $\alpha<\omega_{1}$ hagamos $U_{\alpha}=\{x \in X: x(\alpha)=1\}$.

Si $U$ es un subconjunto abierto y cerrado de $X$ entonces $\chi_{U}$ es la función característica $U$, es decir, $\chi_{U}(x)=1$ para toda $x \in U$ y $\chi_{U}(x)=0$ si $x \notin U$. El espacio $X$ es cero-dimensional y $\psi(a, X)=\omega$ así que podemos encontrar una familia $\left\{W_{n}: n \in \omega\right\}$ de subconjuntos abiertos y cerrados en $X$ tal que 
$W_{n+1} \subset W_{n}$ para cada $n \in \omega$ y $\bigcap_{n \in \omega} W_{n}=\{a\}$. Definimos $\mathcal{W}=\left\{W_{n}: n \in\right.$ $\omega\}$ y $\mathcal{U}_{n}=\left\{U_{\alpha} \backslash W_{n}: \alpha<\omega_{1}\right\}$ para cada $n \in \omega$. Resulta sencillo verificar que $\mathcal{U}_{n}$ es una familia punto-finita de subconjuntos abiertos y cerrados de $X$ y la familia $\mathcal{V}=\mathcal{W} \cup \bigcup_{n \in \omega} \mathcal{U}_{n}$ es $T_{0}$-separadora en $X$. Definimos $u(x)=0$ para todo $x \in X$; es rutinario verificar que $K_{n}=\left\{\chi_{U}: U \in \mathcal{U}_{n}\right\} \cup\{u\}$ es un subconjunto compacto de $C_{p}(X)$ para cualquier $n \in \omega$. Por eso el conjunto $P=\left\{\chi_{V}: V \in \mathcal{V}\right\} \cup\{u\}$ es $\sigma$-compacto y separa los puntos de $X$.

Llamemos $Q$ al álgebra generada por el conjunto $\mathcal{P}$. Tenemos que $Q$ es $\sigma$-compacto y denso en $C_{p}(X)$. Finalmente observemos que $t(X)>\omega$ porque $a \in \bar{S}$ pero ningún subconjunto numerable de $S$ contiene al punto $a$ en su cerradura. Puesto que $t(X) \leq l\left(C_{p}(X)\right)$ para cualquier espacio $X$ (ver $[$ Ar2, Theorem I.4.1]), podemos concluir que $C_{p}(X)$ no es Lindelöf.

Corolario 2.4.9. Existe un espacio $\sigma$-compacto $X$ tal que $C_{p}(X)$ no es Lindelöf pero tiene una cubierta conservativa de sus subespacios $\sigma$-compactos. 


\subsection{Juegos topológicos en $C_{p}(X)$}

Si un espacio $Z$ tiene una cubierta conservativa compacta entonces se puede definir un juego topológico en él de manera natural; en tal juego, el primer jugador tiene una estrategia ganadora. Así, el estudio de juegos análogos en espacios de funciones abre la posibilidad de fortalecer algunos de los resultados de los capítulos anteriores. La siguiente definición nos describe una ligera variación del juego presentado por R. Telgársky en [Te].

Definición 2.5.1. En un espacio $Y$, consideremos una familia $\mathcal{C} \subset \exp (Y)$. Definimos el juego $\mathcal{G}(\mathcal{C}, Y)$ para dos jugadores I y II quienes alternan jugadas de la siguiente manera: en la jugada número $n$, el jugador $I$ elige $C_{n} \in \mathcal{C}$ y el jugador II elige un conjunto $U_{n} \in \tau\left(C_{n}, Y\right)$. El juego termina cuando se ha realizado la $n$-ésima jugada para cada $n \in \omega$. El jugador I gana si $Y=\bigcup\left\{U_{n}: n \in \omega\right\}$; en caso contrario el ganador es el jugador II.

Definición 2.5.2. Una estrategia t para el primer jugador en el juego $\mathcal{G}(\mathcal{C}, X)$ sobre un espacio $X$ se define inductivamente de la siguiente manera. Primero se elige un conjunto $t(\varnothing)=F_{0} \in \mathcal{C}$. Un conjunto abierto $U_{0} \in \tau(X)$ se llama adecuado si $F_{0} \subset U_{0}$. Para cada conjunto adecuado $U_{0}$ se define un conjunto $t\left(U_{0}\right)=F_{1} \in \mathcal{C}$. Supongamos que se han definido sucesiones adecuadas finitas $\left(U_{0}, \ldots, U_{i}\right)$ y conjuntos $t\left(U_{0}, \ldots, U_{i}\right) \in \mathcal{C}$ para cada $i \leq n$. La sucesión finita $\left(U_{0}, \ldots, U_{n+1}\right)$ es adecuada siempre que la sucesión $\left(U_{0}, \ldots, U_{i}\right)$ lo es también para cada $i \leq n$ y $F_{n+1}=t\left(U_{0}, \ldots, U_{n}\right) \subset U_{n+1}$. Una estrategia $t$ para el jugador I es ganadora si asegura la victoria de este jugador en cada partido en el cual I la utiliza.

Definición 2.5.3. Una estrategia s para el jugador II en el juego $\mathcal{G}(\mathcal{C}, X)$ sobre un espacio $X$ es simplemente una función que a cada sucesión finita $\left(F_{0}, \ldots, F_{n}\right)$ de elementos de $\mathcal{C}$ le asigna un conjunto $U \in \tau\left(F_{n}, X\right)$. Una tal estrategia para el jugador II es ganadora si le asegura la victoria en cada partida en que II la usa.

Teorema 2.5.4. Dado un espacio no vacío $X$, supongamos que $Y=C_{p}(X, \mathbb{I})$ (o $\left.Y=C_{p}(X)\right)$ y $\mathcal{F}=\left\{F \subset Y: F\right.$ es denso en ninguna parte en $\left.C_{u}(X, \mathbb{I})\right\}$ (o $\mathcal{F}=\left\{F \subset Y: F\right.$ es denso en ninguna parte en $\left.\left.C_{u}(X)\right\}\right)$. Entonces el jugador II tiene una estrategia ganadora en el juego $\mathcal{G}(\mathcal{F}, Y)$.

Demostración. Recordemos que si tenemos una función $f \in C(X, \mathbb{I})$ (o $f \in C(X))$ y $\varepsilon>0$ entonces el conjunto $I(f, \varepsilon)=\{g \in Y:|g(x)-f(x)| \leq \varepsilon$ 
para todo $x \in X\}$ es cerrado en el espacio $Y$. Definimos inductivamente una estrategia ganadora $s$ para el jugador $I I$ en el juego $\mathcal{G}(\mathcal{F}, Y)$ sobre $Y$ de la siguiente manera: supongamos que $F_{0} \in \mathcal{F}$ es la primera jugada del jugador $I$. Tomemos cualquier bola abierta $B_{0}$ de radio 1 en $C_{u}(X, \mathbb{I})$ (o en $C_{u}(X)$ respectivamente). Entonces $\varnothing \neq\left(B_{0} \backslash F_{0}\right) \in \tau^{*}\left(C_{u}(X, \mathbb{I})\right)$ (o $\varnothing \neq$ $\left.\left(B_{0} \backslash F_{0}\right) \in \tau^{*}\left(C_{u}(X)\right)\right)$ y por lo tanto es posible encontrar $f_{0} \in B_{0} \backslash F_{0}$ y un número real positivo $\varepsilon_{0}<1$ tales que $I\left(f_{0}, \varepsilon_{0}\right) \subset B_{0} \backslash F_{0}$; entonces $F_{0} \subset\left(Y \backslash I\left(f_{0}, \varepsilon_{0}\right)\right) \in \tau^{*}\left(C_{p}(X, \mathbb{I})\right)\left(\right.$ o $\left.F_{0} \subset\left(Y \backslash I\left(f_{0}, \varepsilon_{0}\right)\right) \in \tau^{*}\left(C_{p}(X)\right)\right)$. Como consecuencia, es posible definir $U_{0}=s\left(F_{0}\right)=Y \backslash I\left(f_{0}, \varepsilon_{0}\right)$ como la primera elección de $I I$.

Vamos a suponer que para cada $i<n$ y cada sucesión finita $\left(F_{0}, \ldots, F_{i}\right)$ de elementos de la familia $\mathcal{F}$ seleccionados por el jugador $I$, hemos definido un conjunto $U_{i}=s\left(F_{0}, \ldots, F_{i}\right)$ y una bola abierta $B_{i}$ de radio no mayor que $2^{-i}$, junto con un número real positivo $\varepsilon_{i}<2^{-i}$ y una función $f_{i} \in Y \backslash F_{i}$ tales que $I\left(f_{i}, \varepsilon_{i}\right) \subset B_{i} \subset I\left(f_{k}, \varepsilon_{k}\right) \subset B_{k}$ y $U_{i}=Y \backslash I\left(f_{i}, \varepsilon_{i}\right)$ para cualesquiera $k<i<n$.

Llamemos $F_{n}$ a la jugada número $n$ del jugador $I$. Tomemos cualquier bola abierta $B_{n}$ de radio no mayor que $2^{-n}$ en $C_{u}(X, \mathbb{I})$ (o en $C_{u}(X)$ respectivamente) tal que $B_{n} \subset I\left(f_{n-1}, \varepsilon_{n-1}\right)$. Entonces $\varnothing \neq B_{n} \backslash F_{n} \in \tau^{*}\left(C_{u}(X, \mathbb{I})\right)$ (o $\left.\varnothing \neq B_{n} \backslash F_{n} \in \tau^{*}\left(C_{u}(X)\right)\right)$, y por esto podemos encontrar $f_{n} \in B_{n} \backslash F_{n}$ y un número real positivo $\varepsilon_{n}<2^{-n}$ tales que $I\left(f_{n}, \varepsilon_{n}\right) \subset B_{n} \backslash F_{n}$. El conjunto $F_{n}$ está contenido en $Y \backslash I\left(f_{n}, \varepsilon_{n}\right)$ por lo que podemos tomar $U_{n}=s\left(F_{n}\right)=Y \backslash I\left(f_{n}, \varepsilon_{n}\right)$ como la jugada número $n$ del jugador $I I$.

Así, la definición de la estrategia $s$ está completa; vamos a convencernos de que es ganadora. Tomemos un partido $P=\left\{\left(F_{n}, U_{n}\right)\right\}_{n \in \omega}$ del juego $\mathcal{G}(\mathcal{F}, Y)$ en el que el jugador $I I$ aplica la estrategia $s$. Por la definición de $s$ tenemos que $U_{n}=s\left(F_{n}\right)=Y \backslash I\left(f_{n}, \varepsilon_{n}\right)$ donde $\varepsilon_{n}<2^{-n}$. Obsérvese que la familia $\left\{I\left(f_{n}, \varepsilon_{n}\right): n \in \omega\right\}$ es decreciente y sus elementos son subconjuntos cerrados no vacíos de espacio métrico completo $C_{u}(X, \mathbb{I}) \quad\left(\right.$ o $\left.C_{u}(X)\right)$, y la correspondiente sucesión de diámetros converge a cero. Esto implica que $\bigcap\left\{I\left(f_{n}, \varepsilon_{n}\right): n \in \omega\right\} \neq \varnothing$ y por lo tanto $\bigcup\left\{U_{n}: n \in \omega\right\} \neq Y$. De modo que el jugador $I I$ gana cada vez que aplica la estrategia $s$.

Observación 2.5.5. Dado un espacio $X$ supongamos que $Y=C_{p}(X, \mathbb{I})$ (o $\left.Y=C_{p}(X)\right)$, y hagamos $\mathcal{F}=\{F \subset Y: F$ es denso en ninguna parte en $\left.C_{u}(X, \mathbb{I})\right\} \quad\left(o \mathcal{F}=\left\{F \subset Y: F\right.\right.$ es denso en ninguna parte en $\left.\left.C_{u}(X)\right\}\right)$. Si $\mathcal{C}$ es una familia de subconjuntos cerrados no vacíos de $Y$ para la cual $I$ tiene una estrategia ganadora en el juego $\mathcal{G}(\mathcal{C}, Y)$ entonces $\mathcal{C} \nsubseteq \mathcal{F}$. 
En lo que resta del presente capítulo estudiaremos el juego $\mathcal{G}(\mathcal{C}, Y)$ donde $\mathcal{C}$ es la familia de todos los subespacios $\sigma$-compactos de ciertos espacios $Y$. Notemos que los elementos de $\mathcal{C}$ no son necesariamente cerrados, así que los resultados de esta sección no se siguen inmediatamente de los resultados en $[\mathrm{Te}]$.

Corolario 2.5.6. Para todo espacio $X$, llamemos $\mathcal{C}$ a la familia de todos los subespacios $\sigma$-compactos de $C_{p}(X)$ que son cerrados en $C_{u}(X)$. Si el jugador $I$ tiene una estrategia ganadora en el juego $\mathcal{G}\left(\mathcal{C}, C_{p}(X)\right)$, entonces el espacio $X$ es discreto.

Demostración. De la Observación 2.5.5 se sigue que algún espacio $\sigma$ compacto $F \subset C_{p}(X)$ cerrado en $C_{u}(X)$ contiene una bola $B(f, r)$ abierta en $C_{u}(X)$. Se sigue que $I\left(f, \frac{r}{2}\right) \subset F$ de lo cual se deduce que $I\left(f, \frac{r}{2}\right)$ es un subespacio $\sigma$-compacto de $C_{p}(X)$ y por lo tanto $C_{p}(X, \mathbb{I})$ lo es también (véase la Proposición 2.2.1). Aplicamos [Tk1, 1.5.3] para concluir que $X$ es discreto.

Lema 2.5.7. Si $\mathcal{C}$ es la familia de todos los subespacios $\sigma$-compactos de $Y=\omega^{\omega}$, entonces el jugador II tiene una estrategia ganadora en el juego $\mathcal{G}(\mathcal{C}, Y)$.

Demostración. Supongamos que $\left(F_{0}, \ldots, F_{n}\right)$ es cualquier sucesión de subespacios $\sigma$-compactos de $Y$. Para el conjunto $F_{n}$ es posible encontrar una familia numerable $\left\{K_{m}^{n}: m \in \omega\right\}$ de subconjuntos compactos de $Y$ tal que $F_{n}=\bigcup_{m \in \omega} K_{m}^{n}$. Para cada $m \in \omega$, el mapeo $\pi_{m}: Y \rightarrow \omega$ es la proyección natural del espacio $Y$ sobre el factor determinado por $m$.

Definimos el conjunto finito $C_{n}^{n}=\bigcup_{i=0}^{n} \pi_{n}\left(K_{i}^{n}\right)$; sea $b_{n}^{n}=\sup C_{n}^{n}+1$. Para cada $m>n$ fijémonos en el número $b_{m}^{n}=\sup \pi_{m}\left(K_{m}^{n}\right)+1$. Dado $m \geq n$ hagamos $V_{m}^{n}=\left\{0, \ldots, b_{m}^{n}-1\right\} \times \omega^{\omega \backslash\{m\}}$. Observemos que $K_{i}^{n} \subset V_{n}^{n}$ siempre que $i \leq n$ y $K_{j}^{n} \subset V_{j}^{n}$ para todo $j>n$. Además si $y \in V_{m}^{n}$ entonces $y(m)<b_{m}^{n}$ para todo $m \geq n$. Definamos $t\left(\left(F_{0}, \ldots, F_{n}\right)\right)=U_{n}=\bigcup_{m \geq n} V_{m}^{n}$.

Para verificar que $t$ es una estrategia ganadora, tomemos un partido $\mathcal{P}=$ $\left\{\left(F_{n}, U_{n}\right)\right\}_{n \in \omega}$ en el que el jugador $I I$ usa la estrategia $t$. Encontraremos un 
punto en $Y \backslash \bigcup_{n \in \omega} U_{n}$. Para todo $n \in \omega$ existe $x_{n} \in Y$ tal que $x_{n}(m)=b_{m}^{n}$ para cada $m \geq n$.

Definimos $y(0)=x_{0}(0)+1$. Ahora, una vez que el valor $y(n)$ ha sido determinado, definimos $y(n+1)=y(n)+\sum_{0}^{n+1} x_{i}(n+1)$. El punto $y$ tiene la propiedad de que para todo $n \in \omega$, si $m \geq n$ entonces $y(m)>x_{n}(m)$ lo que implica que $y \notin U_{n}$. Esto muestra que $Y \neq \bigcup_{n \in \omega} U_{n}$ y por lo tanto el jugador II gana el partido $\mathcal{P}$ por lo cual que $t$ es una estrategia ganadora.

Corolario 2.5.8. Si $X$ es un espacio analítico que no es $\sigma$-compacto y $\mathcal{C}$ es la familia de todos los subconjuntos $\sigma$-compactos de $X$, entonces el jugador II tiene una estrategia ganadora en el juego $\mathcal{G}(\mathcal{C}, X)$.

Demostración. Aplicamos [RJ, Theorem 3.5.3] para verificar que $X$ contiene un subespacio cerrado $Y$ homeomorfo a $\omega^{\omega}$. Por el Lema 2.5.7 el jugador II tiene una estrategia ganadora en el juego $\mathcal{G}(\mathcal{C}, Y)$. Es fácil ver que esto implica que el jugador $I$ I tiene una estrategia ganadora en el juego $\mathcal{G}(\mathcal{C}, X)$.

Corolario 2.5.9. Para todo espacio $X$, llamemos $\mathcal{C}$ a la familia de todos los subespacios $\sigma$-compactos de $C_{p}(X)$. Si el jugador I tiene una estrategia ganadora en el juego $\mathcal{G}\left(\mathcal{C}, C_{p}(X)\right)$, entonces el espacio $X$ es pseudocompacto.

Demostración. Si el espacio $X$ no es pseudocompacto entonces contiene una familia discreta numerable de abiertos no vacíos. Por la Proposición 1.2.2 el espacio $\mathbb{R}^{\omega}$ se encaja como subespacio cerrado de $C_{p}(X)$. Llamemos $\mathcal{C}^{\prime}$ a la familia de todos los subespacios $\sigma$-compactos de $\mathbb{R}^{\omega}$. No es difícil verificar que el primer jugador tiene estrategia ganadora en el juego $\mathcal{G}\left(\mathcal{C}^{\prime}, \mathbb{R}^{\omega}\right)$. Como $\mathbb{R}^{\omega}$ es analítico y no $\sigma$-compacto, tenemos una contradicción con el Corolario 2.5.8.

Corolario 2.5.10. Para todo espacio $X$, llamemos $\mathcal{C}$ a la familia de todos los subespacios $\sigma$-compactos de $C_{p}(X)$ que son cerrados en $C_{u}(X)$. Si el jugador $I$ tiene una estrategia ganadora sobre $C_{p}(X)$ en el juego $\mathcal{G}\left(\mathcal{C}, C_{p}(X)\right)$, entonces el espacio $X$ es finito. 
Demostración. Por el Corolario 2.5.6 el espacio $X$ es discreto, así que podemos aplicar el Corolario 2.5.9 para ver que el espacio $X$ es pseudocompacto y por lo tanto finito. 


\section{Problemas abiertos}

En el caso de que un espacio $C_{p}(X)$ admite una cubierta conservativa cerrada de subespacios con una propiedad $\mathcal{P}$ a menudo sucede que el espacio $C_{p}(X, \mathbb{I})$ tiene la propiedad $\mathcal{P}$. Hemos visto que algunos teoremas clásicos acerca de alguna propiedad $\mathcal{P}$ en $C_{p}(X)$ no se extienden automáticamente a los espacios $X$ tales que $C_{p}(X)$ tiene una cubierta conservativa cerrada cuyos elementos tiene la propiedad $\mathcal{P}$. En particular, no es claro si en estos resultados es posible sustituir $C_{p}(X)$ por $C_{p}(X, \mathbb{I})$. Si la pregunta respectiva de $C_{p}(X, \mathbb{I})$ parece tener interés por sí misma, también la formulamos a continuación.

Problema 1. Supongamos que $X$ es un espacio tal que $C_{p}(X)$ es igual a la unión de una familia conservativa de sus subespacios cerrados de Lindelöf. Sabemos que en este caso $C_{p}(X, \mathbb{I})$ es un espacio de Lindelöf. Sin embargo, ¿es necesariamente $C_{p}(X)$ un espacio de Lindelöf?

Problema 2. Supongamos que $X$ es un espacio tal que $C_{p}(X)$ es igual a la unión de una familia conservativa de sus subespacios cerrados de Lindelöf $\Sigma$. Sabemos que en este caso $C_{p}(X, \mathbb{I})$ es un espacio Lindelöf $\Sigma$. Sin embargo, ¿es necesariamente $C_{p}(X)$ un espacio Lindelöf $\Sigma$ ? La respuesta no es clara ni siquiera para espacios $X$ con un solo punto no aislado.

La presencia de una propiedad topológica en $C_{p}\left(C_{p}(X)\right)$ usualmente implica restricciones en $X$ aún más fuertes que las que se tienen cuando existe esta propiedad en $C_{p}(X)$. Por lo tanto podemos esperar que la siguiente pregunta tenga respuesta afirmativa.

Problema 3. Supongamos que $C_{p}\left(C_{p}(X)\right)$ es la unión de una familia conservativa de sus subespacios cerrados Lindelöf $\Sigma$. ¿Debe ser $C_{p}\left(C_{p}(X)\right)$ Lindelöf $\Sigma$ ? 
Si $C_{p}(X)$ es un espacio Lindelöf $\Sigma$ y tiene la propiedad de Baire entonces $X$ debe ser numerable. Esto motiva la siguiente pregunta.

Problema 4. Supongamos que $X$ es un espacio tal que $C_{p}(X)$ tiene la propiedad de Baire y se puede representar como la unión de una familia conservativa de subespacios cerrados Lindelöf $\Sigma$. ¿El espacio $X$ debe ser numerable?

Si un espacio $X$ tiene spread numerable y $C_{p}(X)$ es un espacio Lindelöf $\Sigma$ entonces $X$ debe ser cósmico. Sin embargo, no es claro si podemos reemplazar $C_{p}(X)$ por $C_{p}(X, \mathbb{I})$ en este resultado.

Problema 5. Supongamos que $X$ es un espacio tal que $s(X) \leq \omega$ y $C_{p}(X)$ tiene una cubierta conservativa cerrada de subespacios Lindelöf $\Sigma$. ¿Tiene el espacio $X$ una red numerable?

Problema 6. Supongamos que $X$ es un espacio tal que $s(X) \leq \omega$ y $C_{p}(X, \mathbb{I})$ es un espacio Lindelöf $\Sigma$. ¿Tiene $X$ una red numerable?

Problema 7. Supongamos que $X$ es un espacio y $C_{p}(X)$ es la unión de una familia conservativa de sus subespacios cerrados $K$-analíticos. Sabemos que en este caso el espacio $C_{p}(X, \mathbb{I})$ es $K$-analítico. ¿Debe $C_{p}(X)$ ser $K$-analítico?

Sabemos que la secuencialidad y la propiedad de Fréchet-Urysohn son propiedades equivalentes en espacios $C_{p}(X)$. Sin embargo, esto no es claro para $C_{p}(X, \mathbb{I})$ así que las siguientes preguntas son obligatorias.

Problema 8. Supongamos que $X$ es un espacio tal que $C_{p}(X)$ es la unión de una familia conservativa de sus subespacios cerrados secuenciales. Sabemos que en este caso el espacio $C_{p}(X, \mathbb{I})$ debe ser secuencial. ¿Debe $C_{p}(X)$ ser secuencial?

Problema 9. Supongamos que $X$ es un espacio tal que $C_{p}(X, \mathbb{I})$ es secuencial. ¿Debe $C_{p}(X, \mathbb{I})$ (o equivalentemente $C_{p}(X)$ ) ser Fréchet-Urysohn?

Se sabe que el $\pi$-peso en $C_{p}(X)$ no se conserva ni por uniones numerables ni por uniones conservativas. La situación no es tan clara si consideramos el peso de $C_{p}(X)$.

Problema 10. ¿Es posible representar al espacio $C_{p}([0,1])$ como unión conservativa de sus subespacios segundo numerables? 


\section{Conclusiones}

Una estrategia que ha probado ser eficiente y existosa para el análisis de los espacios topológicos y los espacios de funciones consiste en descomponer tales espacios en sus subespacios y estudiar estas descomposiciones. Una muestra de lo anterior son los resultados mencionados en la Sección 1.5 que se refieren a descomposiciones numerables de espacios $C_{p}(X)$. En tales resultados no es posible sustituir la hipótesis de que las descomposiciones son numerables por descomposiciones arbitrarias ya que cualquier espacio es trivialmente unión de unipuntuales. En este sentido es posible preguntarse qué tanto es posible debilitar la hipótesis de que la descomposición es numerable en los resultados de la Sección 1.5.

Como se prueba en la Sección 1.5, las cubiertas conservativas son una generalización de las cubiertas cerradas numerables. Esto nos permite plantearnos si es posible generalizar los resultados conocidos para cubiertas numerables de espacios de funciones al caso de cubiertas conservativas. Con esta intención se realizó el estudio de los espacios de funciones del que se desprenden los resultados obtenidos y expuestos en el presente texto. Con base en los resultados del Capítulo 2 concluimos que es posible caracterizar la presencia de una gran variedad de propiedades topológicas en espacios $C_{p}(X)$ por medio del estudio de las descomposiciones en familias conservativas que dichos espacios admiten.

Finalmente, en la Sección 2.5 se muestra que mediante el estudio de los juegos topológicos y las estrategias que se pueden definir en los espacios de funciones también es posible caracterizar propiedades topológicas importantes en dichos espacios.

Los resultados de la tesis dan una respuesta a 8 de las preguntas abiertas publicadas en [Gue]. Además, se generalizan varios resultados publicados de Tkachuk, Arhangel'skii, Casarrubias y Okunev. 


\section{Bibliografía}

[Ar1] A.V. Arhangel'skii, Structure and classifications of topologcal spaces and cardinal invariants (en ruso), Uspehi Mat. Nauk, 33:6(1978), 2984.

[Ar2] A.V. Arhangel'skii, Topological function spaces, Mathematics and its Applications (Soviet Series), 78, Kluwer Acad. Publ., Dordrecht, 1992.

[Ar3] A.V. Arhangel'skii, On Lindelöf property and spread in $C_{p}(X)$-theory, Topology Appl., 74:1(1996), 83-90.

[Ca] F. Casarrubias-Segura, Realcompactness and monolithicity are finitely additive in $C_{p}(X)$, Topology Proc., 24(1999), 89-102.

[Jun] H.J.K. Junnila, Metacompactness, paracompactness, and interiorpreserving open covers, Trans. Amer. Math. Soc., 249:2(1979), 373385.

[En] R. Engelking, General Topology, PWN, Warszawa, 1977.

[GN] J. Gerlits, Z. Nagy Some properties of $C(X)$, I Topol. Appl., 14:2(1982, 151-161.

[GA] J.A. Guthrie, Ascoli Theorems and the pseudocharacter of mapping spaces, Bull. Austral. Math. 191 soc. 10 (1974) 403-408.

[Gr] G. Gruenhage, Spaces having a small diagonal, Topology Appl., 122(2002), 183-200.

[Gue] D. Guerrero Sánchez, Closure-preserving covers in function spaces, Comment. Math. Univ. Carolinae, 51:4(2010), 693-703. 
[GT] D. Guerrero Sánchez, V.V. Tkachuk, Dense subspaces vs closurepreserving covers of function spaces, Top. Proc. 39(2012), 219-234.

[Ho] R. Hodel, Cardinal Functions I, Handbook of Set-Theoretic Topology, Kunen y Vaughan (eds.), Netherlands: Elsevier Science Publishers, 1984, 1-61.

[Ju] I. Juhász, Cardinal Functions in Topology. Ten Years Later, Amsterdam: North Holland P.C., 1980.

[Ju2] I. Juhász, Cardinal Functions in Topology - Ten years later, Mathematical Centre Tracts, 123, Amsterdam, 1980.

[JuS] I. Juhász, Z. Szentmiklóssy, Convergent free sequences in compact spaces, Proc. Amer. Math. Soc., 116:4(1992), 1153-1160.

[Le] A. Leiderman, On properties of spaces of continuous functions, Cardinal Invariants and Mappings of Topological Spaces, Izhevsk Univ. P.H., Izhevsk, 1984, 50-54.

[Mi] E. Michael, K,-spaces, Journ. of Math. and Mech. 15 (1966) 983-1002

[No] N. Noble, The density character of function spaces, Proc. Amer. Math. Sot. 42 (1974) 228-233.

[Ok] O.G. Okunev, On Lindelof $\Sigma$-spaces of continuous functions in the pointwise convergence topology, Topology Appl., 49:2(1993), 149-166.

[Po] H. Potoczny, A nonparacompact space which admits a closurepreserving cover of compact sets, Proc. Amer. Math. Soc., 32:1(1972), 309-311.

[PJ] H. Potoczny, H. Junnila, Closure-preserving families and metacompactness, Proc. Amer. Math. Soc., 53:2(1975), 523-529.

[RJ] C.A. Rogers, J.E. Jayne, K-analytic Sets, C.A. Rogers, J.E. Jayne et al "Analytic Sets", Academic Press, London, 1980, 2-181.

[ST] D.B. Shakmatov, V.V. Tkachuk, When is the space $C_{p}(X) \sigma$-countably compact?, Vestnik Mosk. Univ., Mat., 41:1(1986), 73-75. 
[STe] J.C. Smith, R. Telgarsky, Closure-preserving covers and $\sigma$-products, Proc. Japan Acad., 63:A(1987), 118-120.

[Te] R. Telgársky, Spaces defined by topological games, Fund. Math., 88:3(1975), 193-223.

[Ter] J. Terasawa, Spaces $N U R$ and their dimensions, Topology Appl., 11(1980), 93-102.

[Tka] M.G. Tkachenko, Chains and cardinals (en ruso), DAN SSSR, 238(1978), 773-776.

[Tk1] V.V. Tkachuk, The spaces $C_{p}(X)$ : decomposition into a countable union of bounded subspaces and completeness properties, Topology Appl., 22:3(1986), 241-254.

[Tk2] V.V. Tkachuk, Calibers of spaces of functions and the metrizaton problem for compact subsets of $C_{p}(X)$, Vestnik Mosk. Univ., Mat., 43:3(1988), 25-29.

[Tk3] V.V. Tkachuk, The decomposition of $C_{p}(X)$ into a countable union of subspaces with "good" properties implies "good" properties of $C_{p}(X)$, Trans. Moscow Math. Soc., 55(1994), 239-248.

[Tk4] V.V. Tkachuk, Behavior of the Lindelöf property in iterated function spaces, Topology Appl., 107(2000), 297-305.

[Tk5] V.V. Tkachuk, Lindelöf $\Sigma$-spaces: an omnipresent class, Rev. R. Acad. Cienc. Exactas Fís. Nat. Ser. A Math. RACSAM 2:104(2010), 221-244.

[Tk6] V.V. Tkachuk, A $C_{p}$-Theory Problem Book, Topological and Function Spaces, Springer, New York, 2011.

[TY] T. Terada, Y. Yajima, Closure-preserving covers by nowhere dense sets, Bull. Polish Acad. Sci., 42:1(1994), 21-27.

[Ya] N.N. Yakovlev, On bicompacta in $\Sigma$-products and related spaces, Comment. Math. Univ. Carolinae, 21:2(1980), 263-283. 